Portland State University

PDXScholar

2-13-1996

\title{
Nonlinearities in the Base Emitter Junction of Heterojunction Bipolar Transistors
}

Oliver Woywode

Portland State University

Follow this and additional works at: https://pdxscholar.library.pdx.edu/open_access_etds

Part of the Electrical and Computer Engineering Commons Let us know how access to this document benefits you.

Recommended Citation

Woywode, Oliver, "Nonlinearities in the Base Emitter Junction of Heterojunction Bipolar Transistors" (1996). Dissertations and Theses. Paper 5208.

https://doi.org/10.15760/etd.7084

This Thesis is brought to you for free and open access. It has been accepted for inclusion in Dissertations and Theses by an authorized administrator of PDXScholar. Please contact us if we can make this document more accessible: pdxscholar@pdx.edu. 


\section{THESIS APPROVAL}

The abstract and thesis of Oliver Woywode for the Master of Science in Electrical and Computer Engineering were presented February 13, 1996, and accepted by the thesis committee and the department.

COMMITTEE APPROVALS:

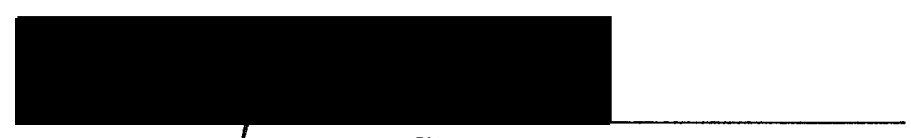

Branimir Pejčinovic, Chair

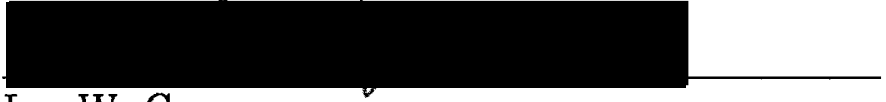

Lee W. Casperson

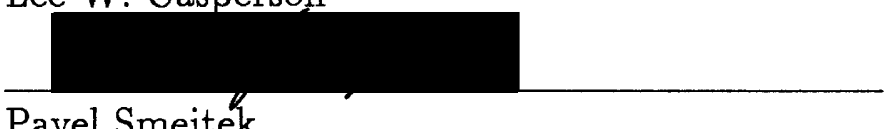

Pavel Smejtek

Representative of the Office of Graduate Studies

DEPARTMENT APPROVAL:

Rolf Schaumann, Chair

Electrical and Computer Engineering

$* * * * * * * * * * * * * * * * * * * * * * * * * * * * * * * * * * * * * * * * * * * * * * * * * * * * *$

ACCEPTED FOR PORTLAND STATE UNIVERSITY BY THE LIBRARY

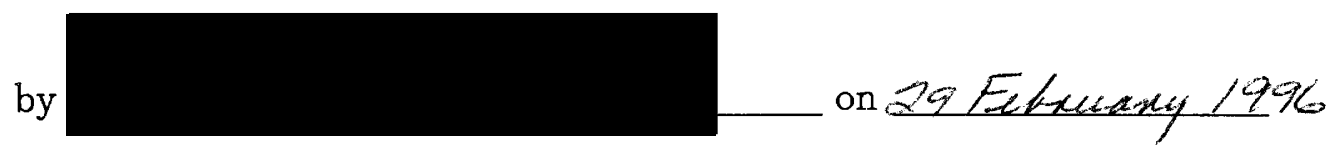




\section{ABSTRACT}

An abstract of the thesis of Oliver Woywode for the Master of Science in Electrical and Computer Engineering presented February 13, 1996.

Title: Nonlinearities in the Base Emitter Junction of Heterojunction Bipolar Transistors

The nonlinear behaviour of the base emitter junction in HBTs is investigated. Nonlinearities cause troublesome distortion and intermodulation of signals and raise the bit error rate. They are therefore a key issue in microwave communication systems.

Hewlett-Packard's Microwave Design System (MDS) software package has been used to simulate these phenomena. The simulation results are verified by an analytical method called nonlinear current method which is a derivative of the Volterra series approach. With the aid of this method new analytical expressions are derived that provide insight into the subtleties of nonlinear phenomena. These expressions are evaluated by the program MAPLE and subsequently compared with the MDS results. Two different models for the B-E junction are juxtaposed. The derived equations reveal the identity and correspondence between the two models.

Finally, this thesis also addresses harmonic balance simulation which is the type of simulation MDS employs to simulate nonlinear circuits. 
Nonlinearities in the Base Emitter Junction of Heterojunction Bipolar Transistors

\author{
by \\ OLIVER WOYWODE
}

A thesis submitted in partial fulfillment of the requirements for the degree of

\author{
MASTER OF SCIENCE \\ in \\ ELECTRICAL AND COMPUTER ENGINEERING
}

Portland State University

1996 


\section{Contents}

List of Figures iv

List of Tables $\quad$ vii

Introduction viii

1 Brief Introduction to Nonlinearity 1

1.1 Two-terminal and Transfer Nonlinearities . . . . . . . . . . . . . 3

1.2 Stability and Nonlinearity . . . . . . . . . . . . . 4

1.3 Substitution Theorem . . . . . . . . . . . . . . . 4

2 Distortion 6

2.1 Linear Distortion . . . . . . . . . . . . . . . . 6

2.2 Nonlinear Distortion . . . . . . . . . . . . . . . 7

2.2.1 Harmonic Distortion . . . . . . . . . . . . . . 7

2.2.2 Intermodulation and Crossmodulation Distortion . . . . . 8

2.2.3 Second and Third Order Intercept Point . . . . . . . . . 10

2.2 .4 1-dB Gain Compression Point . . . . . . . . . . . . . 14

3 Harmonic Balance simulation 15

3.1 Steady-state . . . . . . . . . . . . . . . . . 15

3.2 Harmonic Balance vs. Steady-state . . . . . . . . . . . . . 16

4 Second Order Current Cancellation $\quad 19$

4.1 Outline . . . . . . . . . . . . . . . . . . . . . . . . 19

4.2 Nonlinearities and Volterra Series Method for HBTs . . . . . . . . . 22

4.3 Modelling the HBT . . . . . . . . . . . . . . . . . . . . 24

4.4 Theoretical Explanation of Current Cancellation . . . . . . . . . . 27

4.5 Nonlinear Current Method . . . . . . . . . . . . . . . . . . . . 34

4.6 Taylor Series Expansion of a Nonlinear Conductance . . . . . . . . 37

4.7 Taylor Series Expansion of a Nonlinear Capacitance . . . . . . . . . . 38

4.8 Introducing the Cancellation Term . . . . . . . . . . . . . 39

4.9 Case 1 - Solving the RC Input Circuit by means of the Nonlinear

Current Method . . . . . . . . . . . . . . . . . . . 40 
4.9 .1 Cancellation in terms of Phase . . . . . . . . . . . 42

4.9.2 Impact of Amplitude on Cancellation . . . . . . . . . . . 46

4.10 Case $2 \ldots \ldots \ldots$. . . . . . . . . . . . . . . . 49

4.10 .1 Current Cancellation . . . . . . . . . . . . . 52

4.11 Case $3 \ldots \ldots \ldots \ldots \ldots \ldots$

4.11 .1 Low Frequency . . . . . . . . . . . . . . 57

4.11 .2 Complex $\alpha \ldots \ldots \ldots \ldots \ldots \ldots$

4.11 .3 Current Cancellation . . . . . . . . . . . . . 61

4.12 Equivalence of Case 2 and Case $3 \ldots \ldots \ldots 6$

4.13 Case $4 \ldots \ldots \ldots \ldots \ldots \ldots$

4.13 .1 Nodal Voltages . . . . . . . . . . . . . . . 65

4.13 .2 Terminal Voltages . . . . . . . . . . . . . . . . 66

4.13 .3 Current Cancellation . . . . . . . . . . . . . . 70

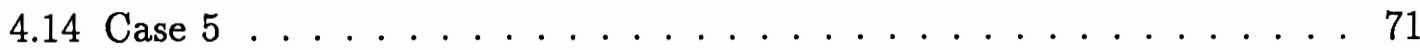

4.14 .1 Low frequency . . . . . . . . . . . . . . . 71

4.14 .2 Complex $\alpha \ldots \ldots \ldots \ldots \ldots$. . . . . . . . . . . . . . . . . .

4.15 Influence of various Parameters . . . . . . . . . . . . . . 74

4.15 .1 Impact of $R_{e e} \ldots \ldots \ldots \ldots \ldots \ldots \ldots$

4.15.2 Impact of $\alpha$ on Phase Difference . . . . . . . . . . . 78

4.16 IP2 . . . . . . . . . . . . . . . . . . . 80

4.17 Review of Approximations . . . . . . . . . . . . . . 82

4.18 Checking the Basic Equation of the Nonlinear Current Method . . . . 84

4.18.1 Disagreement between MDS and MAPLE . . . . . . . . 84

5 Full IP3 Simulation in MDS $\quad \mathbf{8 7}$

5.1 The Self Defined Device in MDS Harmonic Balance Simulations . . . 87

5.2 Weighting Function . . . . . . . . . . . . . . . . . . . . . 89

5.2 .1 Convolution Integral . . . . . . . . . . . . 90

5.2 .2 Double Convolution Integral _. . . . . . . . . . . 90

5.3 Simulating the IP3 . . . . . . . . . . . . . . . . . . . . 90

5.3.1 Implementing the Nonlinear Conductance . . . . . . . . . . 92

5.3.2 Implementing the Nonlinear Capacitance . . . . . . . . . . . . 94

5.3.3 Final Simulation Setup . . . . . . . . . . . . . . . 99 94

6 Conclusion 99

6.1 Next Steps . . . . . . . . . . . . . . . . . . . . . . . . 101

6.1.1 Including the Base Collector Junction . . . . . . . . . . . 101

6.1.2 More Physical Based Model of the Base Emitter Junction . . . 101

$\begin{array}{ll}A \text { Decibels and nepers } & 102\end{array}$

B MAPLE Calculation of $\left(V_{x} \cos m \omega_{x} t+\cdots\right)^{n} \quad 104$ 
C MAPLE Taylor Series Expansion of a Nonlinear Conductance 107

D MAPLE Taylor Series Expansion of Nonlinear Capacitance 111

E MAPLE Verification of Phase Difference 115

F MAPLE Nonlinear Current Method Case $5 \quad 118$

G Time Waveform in MDS $\quad 122$

$\begin{array}{lr}\text { Bibliography } & 126\end{array}$ 


\section{List of Figures}

1.1 Illustrating the substitution theorem $\ldots \ldots \ldots \ldots \ldots$

2.1 The two-tone intermodulation test which is basically a test to measure various output powers vs. input power. It illustrates the geometrical concept of IP2 and IP3. The third order intercept point is found by extending the straight lines as indicated and is equal to $P_{\text {out }}$ at that point. IP2 is defined in the same fashion. . . . . . . . . . . 11

4.1 General equivalent circuit model for HBT with second order current sources for the base emitter junction. This model is the starting point referred to in outline 4.1. The difference of the nodal voltages $\mathrm{vb}$ and ve is the terminal voltage of the nonlinear B-E junction characterized by $g_{1}$ and $c_{1}$ as well as the corresponding current sources $i_{g 2}(t)$ and $i_{c 2}(t)$. Note that $g_{1}, c_{1}, g_{1}, c_{1}$ are the Taylor series expansion coefficients of the large signal relationships of the B-E junction. . . . . . 24

4.2 Equivalent circuit model for case 4 and $5 \ldots \ldots \ldots 25$

4.3 Decoupling the HBT circuit by assuming $\alpha=1 \ldots \ldots \ldots$

4.4 Generation and transfer of the nonlinear B-E voltage $v_{b e} \ldots \ldots \ldots 28$

4.5 If $\alpha=1$ this circuit is decoupled from the circuit shown in Fig. 4.3. This is necessary to explore separately the nonlinear voltage drop which will drive the nonlinear currents through the conductance. . . . 29

4.6 Simulation setup for the RC input circuit. . . . . . . . . . 30

4.7 Spectral lines at the fundamental $(10 \mathrm{GHz})$, the second harmonic $(20$ $\mathrm{GHz}$ ) and the third harmonic $(30 \mathrm{GHz})$ of $V_{b e}$. The marker values denote the peak amplitude value of the associated waveform. . . . . 31

4.8 Time waveform of the spectral lines in Fig. $4.7 \ldots \ldots . \ldots 32$

4.9 Phase variation with frequency for case $1 \ldots \ldots \ldots$

4.10 Circuit for case 2. . . . . . . . . . . . . . . . . . 49

4.11 The overshoot of $\phi_{2}$ immediately before the fall-off is mainly responsible for the deviation of $\phi_{\text {diff }}$ from $\pi \ldots \ldots \ldots \ldots$ 
4.12 The marker ig1, ig2 and ie denote the time waveforms of the phasors $I_{g 1}(2 \omega), I_{g 2}(2 \omega)$ and $I_{e}(2 \omega)$ in the frequency domain, respectively and have been set at $\mathrm{t}=9.09$ ps. $I_{g 1}(2 \omega)$ and $I_{g 2}(2 \omega)$ are the currents through the linear $\left(g_{1}\right)$ and the nonlinear part $\left(g_{2}\right)$ of the nonlinear conductance g. $I_{e}(2 \omega)$ is the emitter current $\left(i_{g 1}(t)+i_{g 2} t\right)=i_{e}(t)$,

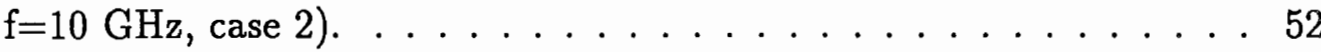

4.13 The corresponding phasor diagram to the time waveforms in Fig.4.12

4.14 The reduced emitter current is due to better cancellation and due to its definition, namely that more current flows through the B-E capacitance at higher frequencies $\left(I_{g 1}(2 \omega)+I_{g 2}(2 \omega)=I_{e}(2 \omega), \mathrm{f}=50\right.$ $\mathrm{GHz}$, case 2).

4.15 Equivalent circuit model for case 3. Note the definition of emitter current which entails a complex $\alpha$ as given in eq. 4.52 .

$4.16 I_{g 1}(2 \omega)+I_{g 2}(2 \omega) \approx I_{e}(2 \omega)$. The marker ig1, ig2 and ie of the time waveform correspond to its counterparts in the frequency domain $I_{g 1}(2 \omega), I_{g 2}(2 \omega)$ and $I_{e}(2 \omega)$, respectively, which is just a different way of stating the same as in the caption of Fig. 4.12. Practically speaking, $I_{g 1}(2 \omega)+I_{g 2}(2 \omega)=I_{e}(2 \omega)$, because the magnitude of the currents steming from the B-E capacitance is small compared to the currents in the onductive portion. But since this model explicitely defines the emitter current through conductance and capacitance one might object that Kirchhoffs current law is not satisfied if the current flowing through the capacitor is neglected and an equal sign is used for the above relationship. Therefore the $\approx$ sign has been used in the caption of Fig.4.16 for the sake of consistency. Case3 . . . . . . . . . . 62

4.17 Equivalent circuit model for case $4 \ldots \ldots$. . . . . . . . 6 65

4.18 The negative feedback of $R_{e e}$ reverses the frequency impact on the phase shift for the nodal voltages with respect to case 2, Fig. 4.11. . . 67

4.19 The crucial difference to case 2 is that $R_{e e}$ eliminates the overshoot of $\gamma_{2}$ immediately before the fall-off. Accordingly, $\gamma_{\text {diff }}$ is closer to $\pi$ in this range.

4.20 Observe the greatly reduced magnitude of the nonlinear currents flowing through the B-E conductance due to $R_{e e}$ in case 4 with respect to case 2 and $3\left(I_{g 1}(2 \omega)+I_{g 2}(2 \omega)=I_{e}(2 \omega), \mathrm{f}=10 \mathrm{GHz}\right.$, case 4$)$. . . 70

4.21 Equivalent circuit model for case 5. Note that the $I_{e}$ is defined through B-E conductance and capacitance. . . . . . . . . . 72

4.22 Phase difference summarized for case 1,2 and $4 \ldots \ldots$. . . . 74

4.23 Impact of $R_{e e}$ on phase difference . . . . . . . . . . . 75 
4.24 Impact of $R_{e e}$ on cancellation. The marker ig1, ig2 and ie denote the time waveforms of the phasors $I_{g 1}(2 \omega), I_{g 2}(2 \omega)$ and $I_{e}(2 \omega)$, respectively and have been set at $\mathrm{t}=9.09$ ps. $I_{g 1}(2 \omega)$ and $I_{g 2}(2 \omega)$ are the currents through the linear $\left(g_{1}\right)$ and the nonlinear part $\left(g_{2}\right)$ of the nonlinear conductance $g . I_{e}(2 \omega)$ is the emitter current. All currents are at the second harmonic. Case 4 , larger $R_{e e} \ldots \ldots \ldots$. . . 77

4.25 Impact of $\alpha \ldots \ldots \ldots \ldots \ldots \ldots 78$

4.26 IP2 for various models. . . . . . . . . . . . . . . . 81

$5.1 \quad$ SDD $I_{c}=f\left(I_{e}\right) \ldots \ldots \ldots \ldots \ldots \ldots$

5.2 Circuit page showing the simulation setup in MDS . . . . . . 91

5.3 SDD - nonlinear resistor circuit page . . . . . . . . . . . 93

5.4 Symbol page . . . . . . . . . . . . . . . . . . 93

5.5 Scion page . . . . . . . . . . . . . . . . . 94

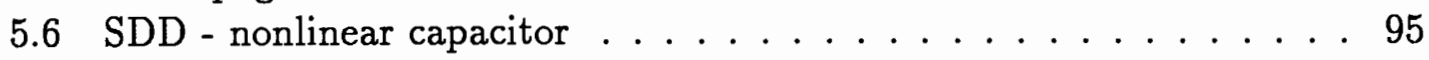

5.72 tone HB simulation . . . . . . . . . . . . . . . 96

5.8 Various IP3 vs frequency . . . . . . . . . . . . . . . 97

G.1 Approximation of a full frequency waveform by 4 harmonic frequencies. 124 


\section{List of Tables}

4.1 Comparison among two MDS simulations which used fitted and calculated taylor coefficients and the program MAPLE to test eq. 4.36. . 45

4.2 Display of fitted and calculated coefficients . . . . . . . . . . 46

4.3 Evaluating the different approximations by comparing the terminal voltage across the $\mathrm{B}-\mathrm{E}$ junction $\ldots \ldots \ldots \ldots$

4.4 Checking approximation . . . . . . . . . . . 85

4.5 Disagreement between MDS and MAPLE . . . . . . . . 86

5.1 Comparison of IP3 in $\mathrm{dBm} \ldots \ldots \ldots \ldots$ 


\section{Introduction}

There is a great deal of interest in operating semiconductor devices at high frequencies and in large-signal mode. The driving force are wireless and telecommunication applications. Even for applications that may have been considered linear in the past, the requirement for low power operation in portable systems drives the circuit designers toward nonlinear modes, such as class $\mathrm{AB}$ and $\mathrm{B}$ operation of amplifiers. In addition, an ever increasing range of frequencies needs to be covered.

An "ideal" device would have small distortion and intermodulation products, as measured by the second and third order intercept points IP2 and IP3, as well as being capable of operation well into the $\mathrm{GHz}$ range. There are many candidates for such a device: GaAs MESFETs, HBTs in GaAs and other materials, and Si based BJTs. A common expectation is that the nonlinear properties of a device are determined by the nonlinearity at its controlling terminal. In this view, one would expect MESFETs to fare better than HBTs due to only a quadratic dependence of output current on gate voltage, which is compared with an exponential dependence of current on base voltage for HBTs. It has been recognized for some time, however, that HBTs and Si BJTs actually perform much better than expected, i.e. they possess very good linear properties, such as IP2 and IP3 as well as low intermodulation products (IMD3). Also, the power added efficiency of HBTs is about twice 
the efficiency of a typical MESFET at the same IP3.

In HBTs the time delay between emitter and collector current is a crucial physical feature. Accordingly, this dynamical effect needs to be addressed by analytical models. Two of the most commonly known analytical models are the Ebers-Moll (EM) and the Gummel-Poon (GP) model. They do not include the time delay, however. Teeter et al. [3] have shown that inclusion of the time delay in the EM model results in excellent agreement between measured and modeled data results. Laser and Pulfrey [13] have shown that the EM and GP model are mathematically equivalent if the time delay is incorporated. HBTs exhibit several significant differences in electrical performance compared to Si BJT's, such as:

- no region of constant current gain,

- base width modulation (Early effect) and conductivity modulation (Webster effect) may be ignored due to high base doping,

- base pushout (Kirk effect) may be of concern if $V_{b e}$ has to be large.

Hence, the challenge lies in developing an HBT model which covers simultaneously dc, small-signal ac, and microwave large-signal applications. Much work has been done in explaining HBT behavior based on an equivalent circuit approach $[7,6,5$, 18]. There are different approaches, for example Wang et al. [5] undertook involved simulation based on a SPICE model. Unfortunately, the SPICE simulator cannot consider the frequency domain. It is therefore impossible to model the time delay. Samelis et al. [18] used the most common model regarding the current gain and 
its time delay. They basically focused on cancellation among currents induced by B-E and B-C junction. Maas and Nelson [6] suggested an interesting and simple model for the current gain which automatically includes the gain roll-off at higher frequencies. Brazil et al. [7] proposed a model which differs from common models mainly in that the base region is split into several nodes and that two diodes with different charachteristics simulate $I_{b}$ and $I_{c}$ independently.

Volterra series approach is the analytical tool to tackle nonlinearities in devices or circuits while harmonic balance simulation is the numerical tool used for the same purpose. Applying Volterra series to specific HBT models extends them into the nonlinear range without having to perform time domain simulation.

Generally, device models may be divided into numerical models based on the Boltzmann Transport Equation its moments and analytical models based on passive lumped elements equivalent circuit models. While the latter are easier and faster to solve by computers, they sometimes do not properly reflect the physics of the device. On the other hand, physical models are accurate but computationally expensive. This calls for a trade-off to develop circuit models whose parameters mirror the essential device physics and are easy to extract from measurments.

There are analytical and curve-fitting methods as well as a combination of both to extract parameters from measured data. The analytical method is not as accurate as the curve-fitting one. Schaper and Holzapfl [19] cut their HBT into three shells accounting for the intrinsic, extrinsic and connecting parts of the HBT. The equivalent circuit elements are unambiguously evaluated from impedance and admittance representation of measured S-parameters over the frequency range of interest. This 
is particularly important for the exact extraction of physically significant HBT parameters such as base and collector transit times [2]. The T-like topology approach allows for reducing all measured data to 15 frequency independent parameters of the small-signal equivalent circuit. The curve-fitting method usually employs an optimizer to minimize an error function, e.g. least-square fitting. The disadvantage is that this method operates in a 15 dimensional mathematical space. Hence the error function has many minima that may make little sense from a physical or circuit application point of view. This problem is circumvented in the analytical method.

It is believed that a sound physical underderstanding of the nonlinear base emitter junction operation can significantly contribute to the development of circuit based HBT models. Sound understanding starts at a basic level; consequently, second order nonlinearities are of major concern in this thesis. 


\section{Chapter 1}

\section{Brief Introduction to Nonlinearity}

A nonlinear device or network produces output signals at frequencies not contained in the input signal. That is, the output signal is distorted with respect to the input signal. The new frequencies are called harmonics and intermodulations (or intermodulation products). The generation of new frequencies also implies that the principle of superposition no longer holds for nonlinear circuits since e.g. $i^{2}=\left(i_{1}+i_{2}\right)^{2} \neq i_{1}^{2}+i_{2}^{2}$.

If a stable nonlinear system is driven by different frequencies, i.e. $\omega_{1}, \omega_{2}, \ldots, \omega_{i}$ then in the general case all possible combinations of frequencies may appear at the output, and their sum is given by:

$$
\sum_{a=-\infty}^{+\infty} \sum_{b=-\infty}^{+\infty} \cdots \sum_{n=-\infty}^{+\infty}\left(a \omega_{1}+b \omega_{2}+\cdots+n \omega_{i}\right)
$$

The number of summations in eq. 1.1 should be equal to the number of input signals with different frequencies. Eq. 1.1 requires the mathematical analysis of nonlinearities not to exclude negative frequencies. Note also that eq. 1.1 suggests that an infinte number of output signals can be generated by the nonlinearity even if only a single frequency is applied (see, e.g., eqs. 2.1, 2.2, 2.3). The output 
spectrum $^{1}$ is discrete, however. Not all combination frequencies may be generated. This depends on the nonlinear transfer characteristic and on the source and load terminations. Nonlinear networks convert the frequency spectrum of the input signal. This conversion is accompanied by a shift of energy in the spectrum and changes the waveform's shape relative to the input signal [11]. The frequencies generated by the nonlinearity are positive, i.e.:

$$
\left(a \omega_{1}+b \omega_{2}+\cdots+n \omega_{i}\right) \geq 0
$$

The analysis of nonlinear networks must nevertheless allow for negative frequencies. Or, in other words, nonlinear networks are described by time relations involving the instantaneous values of current and voltage [1]. Hence, the transfer from the trigonometrical functions to the exponential functions of complex coefficients is not possible by performing the calculations with exponential functions and taking the real part of the end result. This would work only if linear operations were applied during the calulations with exponential functions. For nonlinear networks the transfer from the time function to the exponential function of complex coefficients is performed as follows:

$$
\begin{aligned}
& v(t)=\sqrt{2} V \cos (\omega t+\varphi)=\sqrt{2} V^{+} e^{j \omega t}+\sqrt{2} V^{-} e^{-j \omega t} \\
& V^{+}=\frac{1}{2} V e^{j \varphi} ; V^{-}=\frac{1}{2} V e^{-j \varphi}
\end{aligned}
$$

$\mathrm{V}$ is the root mean square (rms) value, $V^{+}$and $V^{-}$are phasors ${ }^{2}$ (i.e. the coefficients of the exponential functions of positive and negative frequencies). $V^{+}$is equal to

\footnotetext{
${ }^{1}$ The spectrum of a signal gives the amplitudes and phases of the components vs. frequency.

${ }^{2}$ Maas [14] gives a useful relation between phasors and Fourier series in his book on p.471.
} 
the complex conjugate of $V^{-}$i.e.:

$$
V^{+}=\left(V^{-}\right)^{*}
$$

Substituting eq. 1.4 into eq. 1.3 and applying Euler's theorem proves the equality in eq. 1.3 .

\subsection{Two-terminal and Transfer Nonlinearities}

It is important to distinguish between two-terminal and transfer nonlinearities [14]. A two-terminal nonlinearity is for example a nonlinear resistor or capacitor. Its value is a function of one independent variable: the voltage or current at its terminals, called the control voltage or current. In a transfer nonlinearity, such as the collector current which is controlled by the emitter current in $\mathrm{HBTs}^{3}$, the control voltage or current is somewhere in the circuit other than at the element's terminals. The two-terminal nonlinearity is more difficult to handle than the transfer one because its interaction with the circuit is more complex. In a simplified view an excitation voltage generates a few current components in the nonlinear resistor. These current components then enter the circuit and eventually return to the nonlinear resistor and start generating a new set of frquencies. On the other hand, an excitation voltage just needs to be plugged in the nonlinear transer characteristic

\footnotetext{
${ }^{3}$ Note that this example violates the usual quasistatic assumption inherent in the analysis of nonlinear circuits. The quasistatic assumption regards any change of the nonlinearity as instanteneously caused by their control voltage or current. Any change in the emitter current, which is the control current here, affects time delayed the collector current (the transfer nonlinearity). How this time delay is considered in the simulation is shown in chapter 3. Other devices that definitely do not obey the quasistatic assumption are transferred electron devices.
} 
and the corresponding components are readily computed, i.e. the nonlinear element is more insulated from the rest of the circuit.

\subsection{Stability and Nonlinearity}

Due to the complex behavior of nonlinear networks their stability criterion is very difficult to define. A nonlinear device or network is regarded as stable if it cannot sustain oscillations, higher harmonics and intermodulations. Another pragmatic approach considers a nonlinear network as stable if a harmonic balance ${ }^{4}$ analysis converges to a solution [14]. This approach also holds in theory because this analysis perturbs the voltage across nonlinearities and if these perturbations do not cause greater ones, the circuit is stable. This is equivalent to the stability concept in linear circuits. Circuit stability does not gurantee convergence of harmonic balance, or practically speaking, if a circuit is stable, harmonic balance may still fail because of numerical difficulties.

\subsection{Substitution Theorem}

As already mentioned in the introduction, the analytical method to verify the simulation results will be the nonlinear current method. It is therefore useful to introduce the substitution theorem upon which the nonlinear current method is based. In Fig.1.1 two circuits are shown. One presents a nonlinearity obeying the relationship $I=f(V)=g_{1} V+g_{2} V^{2}+g_{3} V^{3}+\cdots$ and the other one presents its

\footnotetext{
${ }^{4}$ Harmonic balance is explained in 3 .
} 
nonlinear equivalent lumped circuit elements. Generally, the substitution theorem
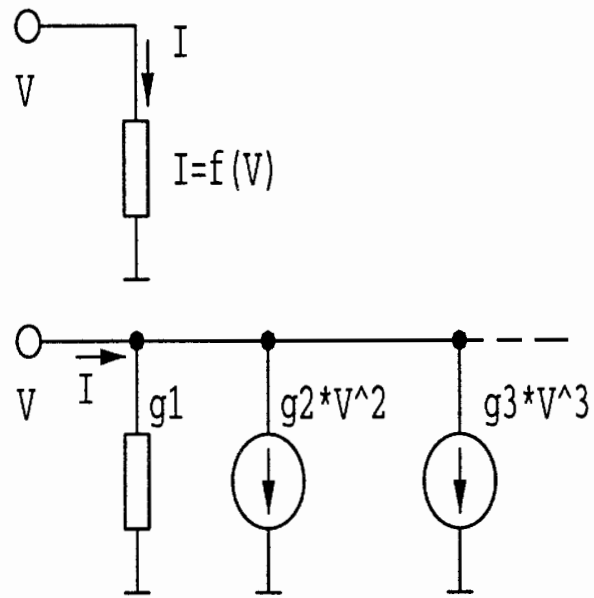

Figure 1.1: Illustrating the substitution theorem

states that a linear or nonlinear resistive circuit element having the characteristic $I=f(V)$ is equivalent to a controlled current source having the same characteristic, wherein $\mathrm{V}$ is the terminal voltage [14]. This theorem also introduces the concept of nonlinear higher order currents, i.e. the terms $g_{2} V^{2}$ and $g_{3} V^{3}$ are the second and third order nonlinear current components, respectively. 


\section{Chapter 2}

\section{Distortion}

As alluded to in chapter 1 an output signal is distorted with respect to the input signal if it possesses frequencies not existing in the input signal because this will alter the shape of the waveform. Generally the transistor's distortion depends on [15]:

- transistor parameters

- load and source terminations

- bias currents and voltages

- frequency

Distortions may be divided into linear and nonlinear distortions [9].

\subsection{Linear Distortion}

Amplitude and phase distortions are linear. They occur if the amplifier's gain differs in magnitude for various frequency components of the input signal or if the 
amplifier's phase shift is not proportional to frequency. Both types change the shape of the output waveform with respect to the input waveform. On the other hand, if the phase shift is proportional to the frequency, the output waveform is delayed (time-shifted). Since the shape of the output waveform is not altered it is not considered as distorted ${ }^{1}$.

In order to avoid linear distortion an amplifier needs to have a constant gain magnitude and a linear (proportional) phase response versus frequency for the range of frequency contained in the input signal.

\subsection{Nonlinear Distortion}

The deviation from the transfer characteristic of an ideal amplifier which is a straight line $e^{2}$ in an output $\left(v_{o}\right)$ vs. input $\left(v_{i}\right)$ plot is referred to as nonlinear distortion. Nonlinear distortion may be divided into harmonic and inter- and crossmodulation distortion.

\subsubsection{Harmonic Distortion}

Suppose the input-output relationship of a nonlinear element is given by a power series:

$$
v_{o}=A_{1} v_{i}+A_{2}\left(v_{i}\right)^{2}+A_{3}\left(v_{i}\right)^{3}+\cdots
$$

\footnotetext{
${ }^{1}$ Therefore this phenomena should not be called distortion. It is nevertheless called as such in the literature.

${ }^{2}$ The line's slope is the gain.
} 
where $A_{1}, A_{2}, A_{3}$ etc. are fitting parameters to satisfy the nonlinear transfer characteristic. If the input voltage is given by:

$$
v_{i}=V_{a} \cos \left(\omega_{a} t\right)
$$

then the output voltage is given by ${ }^{3}$ :

$$
v_{o}=V_{0}+V_{1} \cos \left(\omega_{a} t\right)+V_{2} \cos \left(2 \omega_{a} t\right)+V_{3} \cos \left(3 \omega_{a} t\right)+\cdots
$$

The term $V_{0}$ is a constant, it is the $0^{\text {th }}$ fundamental component and represents a shift in the dc level, $\omega_{a} t$ is the $1^{\text {st }}$ fundamental or $1^{\text {st }}$ harmonic, $2 \omega_{a} t$ is the $2^{\text {nd }}$ fundamental or $2^{\text {nd }}$ harmonic, $3 \omega_{a} t$ is the $3^{\text {rd }}$ fundamental or $3^{\text {rd }}$ harmonic, etc. Note that the dc level shift implies that the excitation of a nonlinearity may offset its dc operating point.

The second- and third-harmonic distortion factors are given by $D_{2}=\frac{V_{2}}{V_{1}}$ and $D_{3}=\frac{V_{3}}{V_{1}}$, respectively: The total harmonic distortion is then given by:

- $D=\sqrt{D_{2}^{2}+D_{3}^{2}+\cdots}$.

\subsubsection{Intermodulation and Crossmodulation Distortion}

Suppose the input voltage consists of two sinusoids of different frequencies and is given by:

$$
v_{i}=V_{a} \cos \left(\omega_{a} t\right)+V_{b} \cos \left(\omega_{b} t\right)
$$

\footnotetext{
${ }^{3}$ The procedure is: substituting eq. 2.2 into eq. 2.1 , recalling that $\left[\cos \left(\omega_{a} t\right)\right]^{2}=\frac{1}{2}\left[1+\cos \left(2 \omega_{a} t\right)\right]$ and $\left[\cos \left(\omega_{a} t\right)\right]^{3}=\frac{1}{4} \cos \left(3 \omega_{a} t\right)+\frac{3}{4} \cos \left(\omega_{a} t\right)$; collecting terms, and defining $V_{0}$ to be equal to the sum of all constant terms, $V_{1}$ to be the sum of the coefficients of the terms with $\omega_{a}, V_{2}$ to be the sum of the coefficients of the terms with $2 \omega_{a}$, etc.
} 
Substituting eq. 2.4 into eq. 2.1 we arrive at:

$$
\begin{aligned}
v_{o} & =A_{1}\left[V_{a} \cos \left(\omega_{a} t\right)+V_{b} \cos \left(\omega_{b} t\right)\right] \\
& +\frac{1}{2} A_{2}\left\{\left(V_{a}\right)^{2}+\left(V_{b}\right)^{2}+\left(V_{a}\right)^{2} \cos \left(2 \omega_{a} t\right)+\left(V_{b}\right)^{2} \cos \left(2 \omega_{b} t\right)\right. \\
& \left.+2 V_{a} V_{b} \cos \left[\left(\omega_{a}+\omega_{b}\right) t\right]+2 V_{a} V_{b} \cos \left[\left(\omega_{a}-\omega_{b}\right) t\right]\right\} \\
& +\frac{1}{2} A_{3}\left\{3\left(V_{a}\right)^{2} V_{b} \cos \left(\omega_{b} t\right)+3 V_{a}\left(V_{b}\right)^{2} \cos \left(\omega_{a} t\right)\right. \\
& \left.+\frac{3}{2}\left(V_{a}\right)^{2} V_{b} \cos \left[\left(2 \omega_{a}-\omega_{b}\right) t\right]+\frac{1}{2}\left(V_{a}\right)^{3} \cos \left(3 \omega_{a} t\right)+\cdots\right\}
\end{aligned}
$$

after some manipulation. It is worthwhile to pause at eq. 2.5 :

- The term $\omega_{a} \pm \omega_{b}$ is called a second-order intermodulation product (IMD2), because the sum of the coefficients of $\omega_{a}$ and $\omega_{b}$ is two.

- The term $2 \omega_{a}-\omega_{b}$ is called a third-order intermodulation product (IMD3), because the sum of the coefficients of $\omega_{a}$ and $\omega_{b}$ is three.

- The terms $\omega_{a} \pm \omega_{b}$ and $2 \omega_{a}-\omega_{b}$ represent the new frequenices not contained in the input signal.

- As the third-order intermodulation terms ${ }^{5}$ are very close to the original input, they can not be filtered out. The input needs to be reduced to keep them at a tolerable level. Hence, the maximum usable output power (or the upper limit of the amplifier's dynamic range) is determined by intermodulation distortion. The lower limit of the amplifier's dynamic range is determined by its noise

\footnotetext{
${ }^{4}$ This concept is ambigous, however. For example, $2 \omega_{a}+\omega_{b}$ could be a fifth order mixing product, namely $\omega_{a}+\omega_{a}+\omega_{a}-\omega_{a}+\omega_{b}$. Generally, it is not possible to determine the mixing product's order from its frequency.

${ }^{5}$ There are also third-order intermodulation terms at $2 \omega_{a}+\omega_{b}$ but they are not close to the fundamental frequencies, hence they are less troublesome as they can be filtered out.
} 
factor. Since the term $2 \omega_{a}-\omega_{b}$ is the most important and it arises from the third-order term $A_{3}$ this intermodulation is often referred to as third-order distortion.

- The term $\frac{1}{2} A_{3}\left\{3\left(V_{a}\right)^{2} V_{b} \cos \left(\omega_{b} t\right)\right\}$ falls into the original frequency band with a significant amplitude. The amplitude $V_{a}$ which belongs to the input frequency $\omega_{a}$ has been transferred to $\omega_{b}$ due to the nonlinearity and interferes with the amplitude $V_{b}$ of the other input frequency $\omega_{b}$. This effect is called cross modulation, or since it is caused by the third-order term $A_{3}$, also thirdorder distortion ${ }^{6}$.

Sometimes the third order intermodulation and third order cross modulation distortion are lumped together and called third order distortion.

\subsubsection{Second and Third Order Intercept Point}

The two-tone intermodulation test measures the degree of distortion and intermodulation produced by an amplifier. In this test two sinusoids of equal amplitude (i.e. $V_{a}=V_{b}$ ) are applied at the amplifier's input. Three grahps ${ }^{7}$ are plotted in Fig.2.1:

\footnotetext{
${ }^{6}$ There are higher order distortions but usually their amplitude is small.

${ }^{7}$ The unit $\mathrm{dBm}$ in Fig.2.1 is explained in appendix A.
} 


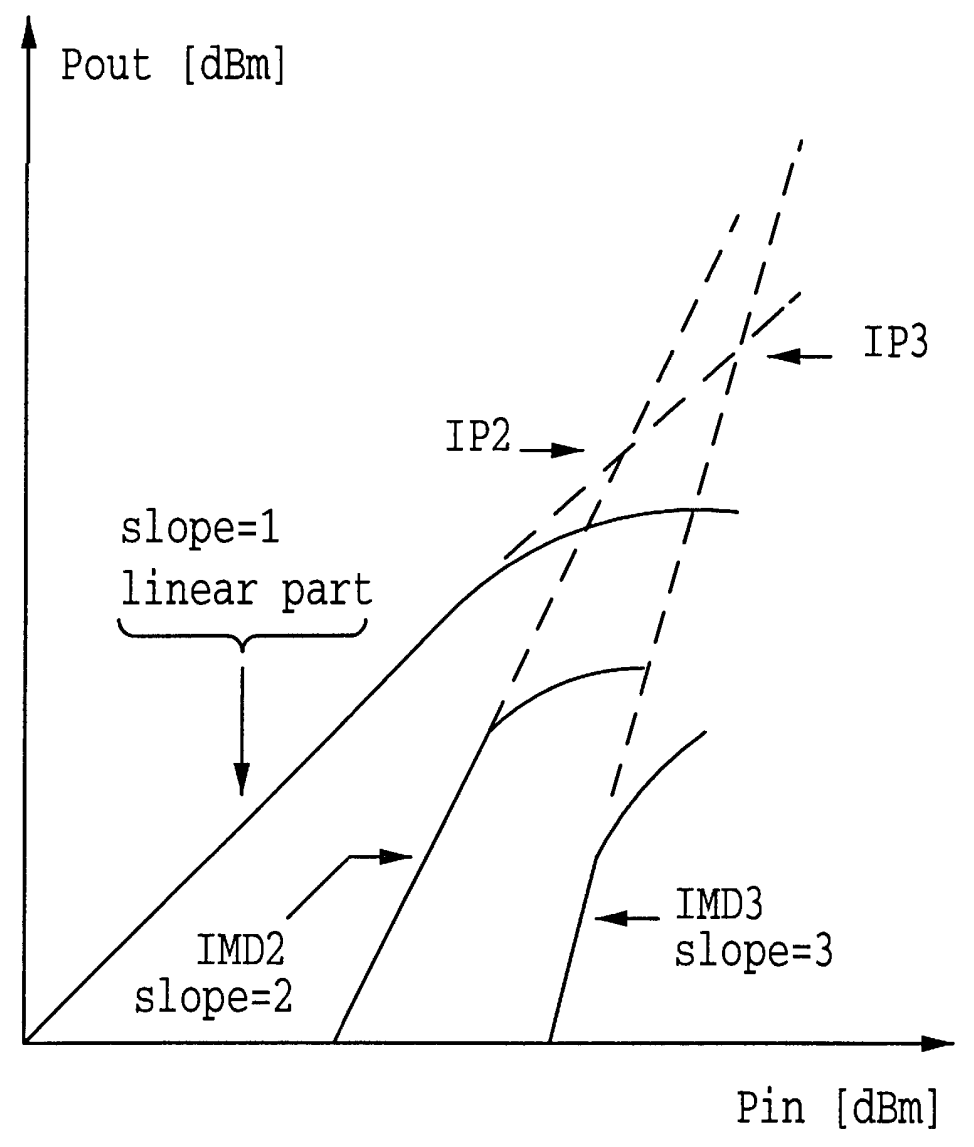

Figure 2.1: The two-tone intermodulation test which is basically a test to measure various output powers vs. input power. It illustrates the geometrical concept of IP2 and IP3. The third order intercept point is found by extending the straight lines as indicated and is equal to $P_{\text {out }}$ at that point. IP2 is defined in the same fashion. 
1. The graph with unity slope describes the linear relationship: desired output power $\left(P_{\omega_{a}}\right)$ vs. input power $\left(P_{i n}\right)$ of the amplifier.

2. The graph with slope 2 denotes the output power at the second harmonic $\left(P_{2 \omega_{a}}\right)$ vs. input power $\left(P_{i n}\right)$. If $\omega_{a}$ and $\omega_{b}$ are separated by a small frequency this graph presents the output power $\left(P_{\omega_{a}+\omega_{b}}\right)$ at the second order intermodulation product IMD2 vs. input power $\left(P_{\text {in }}\right)$ as well.

3. The graph with slope 3 denotes the output power $\left(P_{2 \omega_{a}-\omega_{b}}\right)$ at the third order intermodulation distortion term vs. $P_{i n}$. The slope of 3 follows from the assumed $v_{0}$ in eq. 2.1, i.e. the power of IMD3 is proportional to the cube of the input signal amplitude (recall that $V_{a}=V_{b}$ ).

If the input power is increased far enough, the output power departs from a straight line due to signal clipping. IP3 characterizes the linear, or depending on the point of view, the nonlinear properties of an amplifier. From eq. 2.1 it can be shown that IP3 is roughly $10 \mathrm{~dB}$ above the $1-\mathrm{dB}$ compression point (the meaning of which will be explained later on). That is,

$$
P_{I P 3}(d B m)=P_{1 d B}(d B m)+10 d B
$$

where $P_{I P 3}$ is the power level at the third-order intercept point and $P_{1 d B}$ is the output power at the 1-dB compression point. It can also be shown that

$$
2 P_{2 \omega_{a}-\omega_{b}}=3 P_{\omega_{a}}-2 P_{I P 3}
$$

where $P_{2 \omega_{a}-\omega_{b}}=P_{I M D 3}$ is the output power level at the third order intermodulation distortion product and $P_{\omega_{a}}$ the output power at the fundamental frequency. 
Reshuffling eq. 2.7 gives

$$
P_{I P 3}=\frac{3 P_{\omega_{a}}-P_{I M D 3}}{2},
$$

which illustrates that IP3 is high if IMD3 is low or, practically speaking, the less nonlinear the device the less the distortion and thus the higher IP3. In the region of gain compression the extrapolation may be doubted due to higher order effects. Still, this concept remains useful for comparison in the moderate signal range. The IP3 concept described here applies only to two-tone excitation and the associated intermodulation powers assume that both excitation levels vary simultaneously [14]. The straight line portion in Fig.2.1 may have ripples or even curvature if the nonlinearity has strong reactances. The higher the intercept point the better the amplifier performance. A high intercept point implies a small IMD2 or IMD3 magnitude and therefore less distortion. Traditionally the third order intermodulation distortion may be reduced by decreasing the output power of the amplifier. This, however, reduces its efficiency. The common emitter configuration has lower third order intermodulation distortion than the common base configuration due to the negative feedback and linearization effect from the emitter and base resistances [5]. Recently, it was suggested that the third-order intercept point of an amplifier be improved by reducing its gain by means of matching networks [16]. Others reported that the input matching network has no impact on third order intermodulation distortion [5]. 


\subsubsection{1-dB Gain Compression Point}

The 1-dB gain compression point $\left(G_{1 d B}\right)$ characterizes the nonlinearity of an amplifier as well [8]. It is defined as the power gain where the nonlinearities of the transistor reduce the power gain by $1 \mathrm{~dB}$ over the small-signal linear power gain $G_{0}$.

$$
G_{1 d B}(d B)=G_{0}(d B)-1
$$

Since the power gain is defined as

$$
G_{p}=\frac{P_{\text {out }}}{P_{\text {in }}} \Longrightarrow P_{\text {out }}(d B m)=G_{p}(d B)+P_{\text {in }}(d B m)
$$

we can write the output power at the $1-\mathrm{dB}$ gain compression point $\left(P_{1 d B}\right)$ as

$$
P_{1 d B}(d B m)=G_{1 d B}(d B)+P_{\text {in }}(d B m) .
$$

Substituting eq. 2.9 into eq. 2.11 gives

$$
P_{1 d B}(d B m)-P_{i n}(d B m)=G_{0}(d B)-1
$$

which shows that the 1-dB gain compression point is that point at which output power minus input power equals the small-signal power gain minus $1 \mathrm{~dB}$. 


\section{Chapter 3}

\section{Harmonic Balance simulation}

Because harmonic balance (HB) is the type of simulation MDS uses to simulate nonlinear circuits, a few comments about it are in order. First, however, a brief review on steady-state is given as HB relates to the steady-state of a circuit.

\subsection{Steady-state}

The state of a circuit may be defined by a set of its instantaneous element values [11]. If those values do not recur in a certain time sequence the circuit is governed by a transient process. If those values do recur in a certain time sequence the circuit is governed by a steady-state process. Steady-state processes may be further divided into a dc steady (quiescent) state and a periodic state.

Periodic processes are usually analysed by the Fourier transform. This allows for investigation of nonlinearities by spectral methods in the frequency domain which are applied when the conversion of the signal spectrum is of main interest. In the frequency domain, the nonlinearities are equivalently represented by intermodulation 
distortion sources whose amplitudes and phases are iteratively determined by the linear circuit characteristics and the Fourier or Taylor series coefficients associated with those nonlinearities. Otherwise, if the shape (waveform) of the signal matters, the time domain is preferred. The Fourier transform establishes a unique relation between shape and spectrum.

\subsection{Harmonic Balance vs. Steady-state}

In the MDS [10] simulation program HB performs a frequency-domain simulation. Signals are treated as summations of finite numbers of sine-waves, i.e., as periodic signals. Voltages and currents are calculated in much the same way as time-domain simulators such as SPICE do. But HB must solve for the magnitudes and phases of all spectral lines simultaneously. Thus, it computes the spectrum of each signal and therefore mimics a spectrum analyzer. After the analysis, every node voltage consists of a complex spectrum.

$\mathrm{HB}$ inputs one or more sine-wave stimulus to determine for example conversion loss, distortion, and gain compression at the output. As expected, HB is very efficient if the signals are simple in the frequency domain. Often, an amplifier is driven by a sine wave, and those are easily represented as, e.g., Fourier transforms.

Energy is not allowed to exist continuously throughout the spectrum. It is discretized into ideal spectral lines that are the harmonics of more basic fundamental frequencies and intermodulation products of those fundamental frequencies. HB analysis is also called steady state analysis. In summary, the Hewlett-Packard Mi- 
crowave Nonlinear Simulator (MNS) uses the HB analysis technique to find the steady state solution in the frequency domain.

During a HB simulation the following steps occur:

- The DC operating point of the circuit is calculated.

- A linear AC simulation analyses the linear and passive components in the frequency domain.

- Only nonlinear devices are analyzed in the time domain, in order to give the simulation the most general approach; otherwise the phase information would be lost in the frequency domain. The results are included in the analysis via Fourier transform.

- MNS initially checks every node ${ }^{1}$ in the circuit. Nodes which connect linear elements have responses only at the fundamental frequency (i.e., they do not have harmonics) while nonlinear elements have both fundamental and harmonics. Thus the initially computed error according to Kirchhoff's current and voltage laws (i.e., the sum of the node currents) is $100 \%$. The estimated voltage spectrum is refined until Kirchhoff's current and voltage laws are satisfied. Iterative, matrix-driven techniques are used.

- The final solution is a voltage spectrum which adds up to zero at all nodes in the circuit so that the final iteration indicates an error which approaches zero.

\footnotetext{
${ }^{1}$ From a simplified point of view HB separates the network or circuit into a linear and a nonlinear part and balances out the harmonics at the interface. In other words, HB seeks a set of port voltage waveforms (or harmonic voltage components) that give identical currents in the linear and nonlinear part. This set must then be the solution.
} 
In other words, as the sum of all node currents approaches zero, the analysis has converged, and the harmonics are now balanced for each single node as required for steady state.

One of the main advantages of $\mathrm{HB}$ compared to time domain simulators is that HB employs the frequency domain. This is crucial for implementing the time delay (due to transit time and junction charging effects) between the emitter and the collector current in HBTs. The time delay ${ }^{2}$ of the forward current gain $\alpha$ is most naturally established in the frequency domain and this is easily treated in HB.

\footnotetext{
${ }^{2}$ How to implement the delay in MDS is shown in chapter 5.
} 


\section{Chapter 4}

\section{Second Order Current}

\section{Cancellation}

\subsection{Outline}

In this section a plan is outlined. This will serve as a guide on how the complex nonlinear cancellation mechanism in HBTs works and how it is being investigated.

1. Volterra series method, its application, and limitation regarding the nonlinearities in HBTs are reviewed in section 4.2 with respect to the specific investigation here.

2. A general (meaning it involves the smallest number of approximations) circuit based model for the HBT is suggested in section 4.3. This model is the starting point. It will be simplified step by step and each resulting circuit is shown. The approximations made at each stage are highlighted. 
3. With the aid of the most simplified model, which, by the way, is an RC circuit the cancellation of the base emitter junction is, although somewhat ad-hoc, explained theoretically in section 4.4. To avoid confusion: RC circuit refers here to the input RC circuit consisting of the base resistance $R_{b}$ and the nonlinear B-E capacitance $C_{b e}$ in series. The B-E junction is modeled by a nonlinear conductance and a nonlinear base emitter capacitance in parallel. In order to illustrate graphically the cancellation and also nonlinear effects like distortion, the amplitude of the input signal was intentionaly chosen high. Section 4.4 will also introduce or show the following:

- the nonlinear current method which is derived from Volterra series analysis,

- the Taylor series expansion of a nonlinear conductance and capacitance,

- a so-called cancellation term, involving the Taylor series expansion coefficients $\left(g_{1}, c_{1}, g_{1}, c_{1}\right)$ of the large signal relationships of the B-E junction.

4. As already indicated the general model will be simplified step by step. Each step will be dealt with as a separate case. As mentioned above the lowest level upon which the cancellation in the HBT will be explained is an RC circuit with nonlinear capacitance. This time investigation will use a practical relevant input level. This will be labeled as case 1 and is section 4.9. A specific nonlinear phenomena, for example the impact of amplitude on phase, will be analyzed as well. 
5. Case 2 in section 4.10 makes the step from the RC circuit to the HBT by including a nonlinear conductance which, in parallel ${ }^{1}$ to the nonlinear capacitance, makes up the base emitter junction and a current dependent current generator for the output (collector) current.

6. Case 3 in section 4.11 is the same as case 2 except that a different definition of the emitter current is used which entails a different modeling of the common base current gain $\alpha$.

7. The equivalence and correspondence of case 2 and case 3 will be shown in section 4.12 .

8. Case 4 in section 4.13 eliminates an important approximation with respect to case 2 and 3 . It includes the emitter resistance.

9. Case 5 in section 4.14 is the same as case 4 but, as between case 2 and 3 , a different approach regarding emitter current and current gain is taken. This case will be dealt with briefly as the equivalence between case 2 and 3 is not disturbed by including the emitter resistance.

10. The influence of various parameters like emitter resistance and current gain on the cancellation is illustrated in section 4.15 .

11. How the second order current cancellation results in IP2 is shown in section 4.16 .

\footnotetext{
${ }^{1}$ This is a parallel $\mathrm{RC}$ circuit. This circuit is not to be confused with the $\mathrm{RC}$ input circuit mentioned above.
} 
12. The approximations made at each step are revisited in section 4.17.

13. The assumptions involved in the method of nonlinear currents are explained in section 4.18 .

\subsection{Nonlinearities and Volterra Series Method for}

\section{HBTs}

Nonlinearities and Volterra series method were already mentioned a few times. Now, a brief review is presented with respect to its subsequent application to HBTs. Recently it was suggested that there are four major sources causing nonlinearities in HBTs [18]:

- the base emitter junction capacitance $C_{b e}$, which has a depletion, diffusion, and charge storage (due to band discontinuity) component.

- the base emitter junction conductance $g_{b e}$. Since $g_{b e}=\frac{\partial I_{b}}{\partial V_{b e}}$ and $I_{b}$ depends exponentially on $V_{b e}$, this nonlinearity is sometimes called exponential nonlinearity, which is the major source of distortion in BJT's at low bias current levels [12].

- the base-collector junction capacitance $C_{b c}$.

- the common base current gain $\alpha$.

The nonlinearity comes mainly through their exponential dependence on the baseemitter voltage $V_{b e}$, except for $C_{b c}$ which depends on the collector-base voltage $V_{c b}$. 
Wang [5] reports that $C_{b c}$ must have as low a voltage dependence as possible for linear operation.

The exponential function presents a very strong nonlinearity. Hence, one might be tempted to conclude that HBTs exhibit a strongly nonlinear behavior. Surprisingly, HBTs demonstrate a high linearity at low levels of DC bias power despite these nonlinearities [6].

HBTs need to be modeled as nonlinearities with memory even if a nonlinear conductance or capacitance are memoryless [4]. Due to its internal feedback $\left(C_{b c}\right)$ these memoryless nonlinearities can not be decoupled from the equivalent circuit representing the HBT. Since the relation between emitter and collector current involves a time delay, which brings the memory into play, HBTs need to be modeled as nonlinearities with memory. This requires the application of Volterra series as the analytical tool. The memory property rules out the power series method for the analysis, but many of the power series method limitations apply in Volterra series as well:

- multiple excitations are small and noncommensurate in frequency,

- nonlinearities are weak,

- nonlinearity must be a single valued function, for example for a nonlinear capacitor the voltage must be a single valued function of charge.

The power series method is a special case of Volterra in which a single nonlinear Volterra transfer function is replaced by a product of linear transfer functions. 


\subsection{Modelling the HBT}

An equivalent circuit model, suitable for exploring nonlinear effects up to second order, is shown in Fig. 4.1. This model will be simplified by taking out all para-

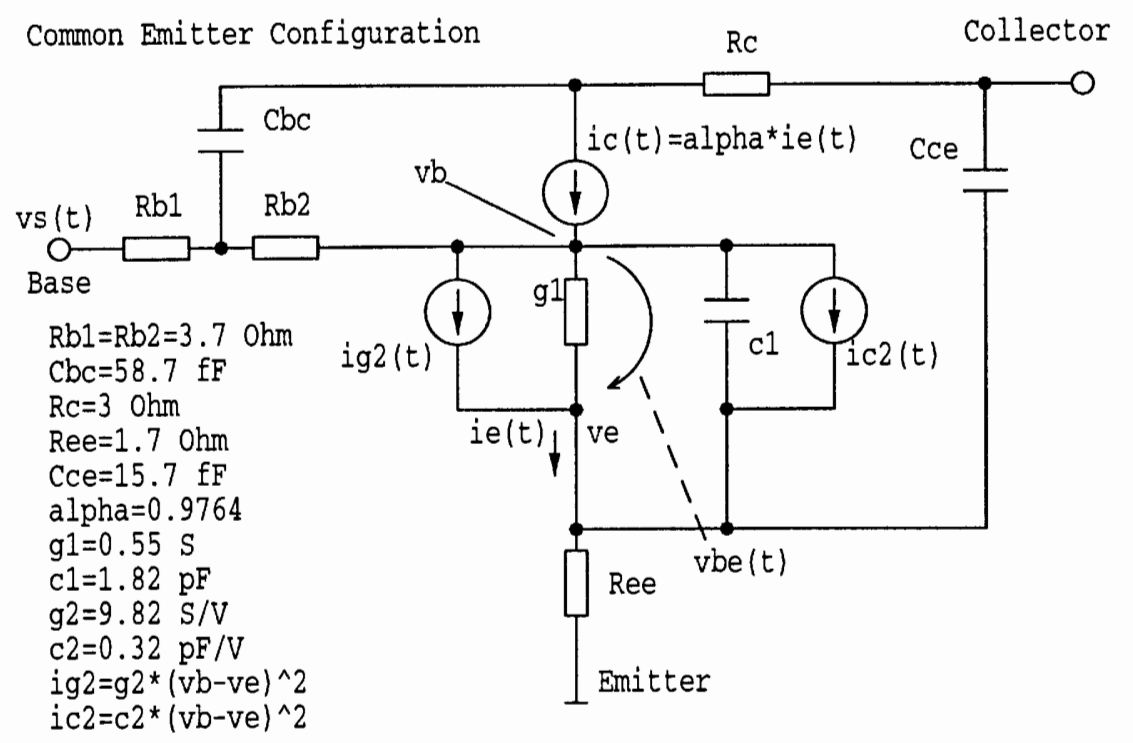

Figure 4.1: General equivalent circuit model for HBT with second order current sources for the base emitter junction. This model is the starting point referred to in outline 4.1. The difference of the nodal voltages $\mathrm{vb}$ and ve is the terminal voltage of the nonlinear B-E junction characterized by $g_{1}$ and $c_{1}$ as well as the corresponding current sources $i_{g 2}(t)$ and $i_{c 2}(t)$. Note that $g_{1}, c_{1}, g_{1}, c_{1}$ are the Taylor series expansion coefficients of the large signal relationships of the B-E junction.

sitics because they make the analysis lengthy and do not actively participate in the cancellation mechanism. The resulting model is shown in Fig. 4.2. This model will be investigated in case 4 and 5 which differ only in definition of the emitter current. The next important approximation is to neglect the emitter resistance. This circuit will be analyzed as case 2 and 3 which differ, as case 4 and 5 , only in definition 


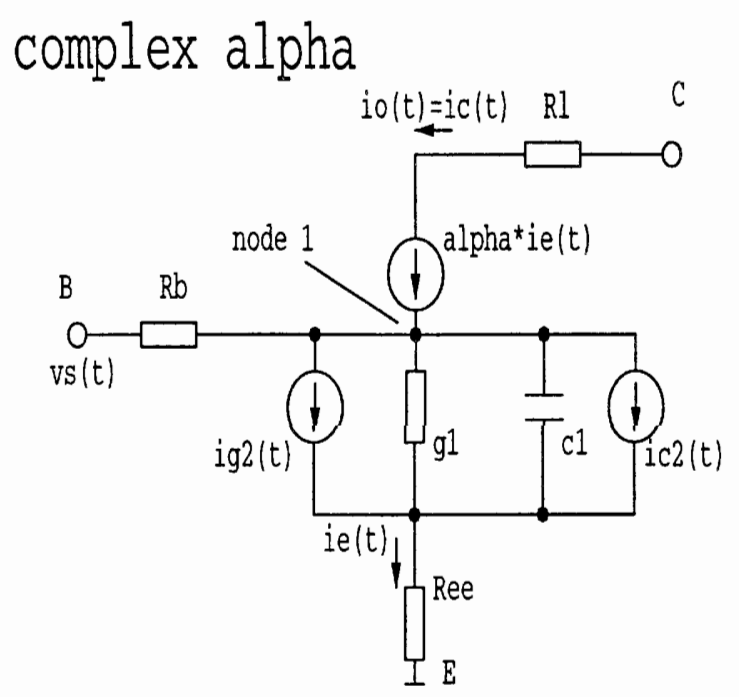

Figure 4.2: Equivalent circuit model for case 4 and 5

of $I_{e}$. The last approximation is to assume a current gain of unity which basically decouples the RC input circuit (series combination of $R_{b}$ and $C_{b e}$ ) and the circuit containing current source $I_{o}=I_{c}=\alpha I_{e}$, as well as the nonlinear base emitter conductance in series. The last two steps of the simplification process are illustrated in Fig. 4.3. The RC input circuit will be examined as case 1 . 


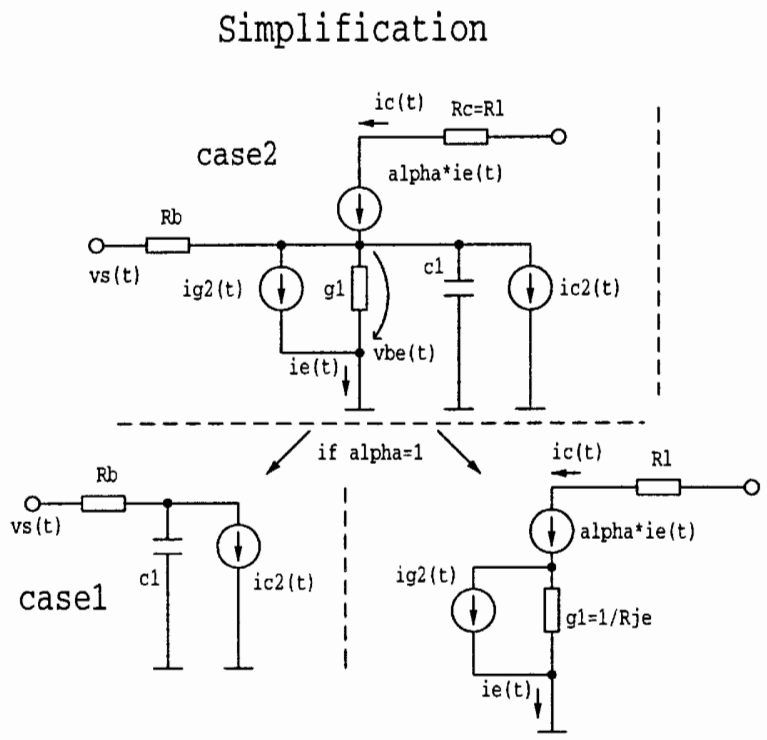

Figure 4.3: Decoupling the HBT circuit by assuming $\alpha=1$ 


\subsection{Theoretical Explanation of Current Cancella-}

\section{tion}

A simple model of a base emitter junction is a conductance and a capacitor in parellel. The cancellation in such a parallel circuit may be stated as follows: The nonlinear voltage drop $v_{b e}$ is caused by the nonlinear base emitter capacitance $C_{b e}$ independently from the nonlinear base emitter conductance $g_{b e}$. Nonlinearity implies that there are many different frequencies contributing to this voltage drop. The voltage drop $v_{b e}$ is instantaneously transferred to $g_{b e}$ and drives nonlinear currents at many different frequencies through the linear portion of $g_{b e}$. In addition, different current components for each frequency are generated because $g_{b e}$ is nonlinear itself. The phase of some of these components is such that they cancel. This simplified approach treats the voltage drop across the capacitor and currents through the conductance separately. This is the underlying reason for the assumption of $\alpha=1$ since this enables us to separate the circuits into two parts. It turns out that this assumption is not too crude. In reality, $\alpha$ is close to one and the currents through the capacitor are small in magnitude compared to the currents through the conductance. This process is illustrated in Fig. 4.4

Let us now turn to the generation of $v_{b e}$. Recall that due to assuming $\alpha=1$, which is identical to assuming $i_{e}=i_{o}=i_{c}$, the circuit in Fig. 4.3 is split into an RC input circuit (shown in Fig. 4.5) and a circuit containing current source $I_{c}$ and the nonlinear base emitter conductance in series. The RC input consists of the 


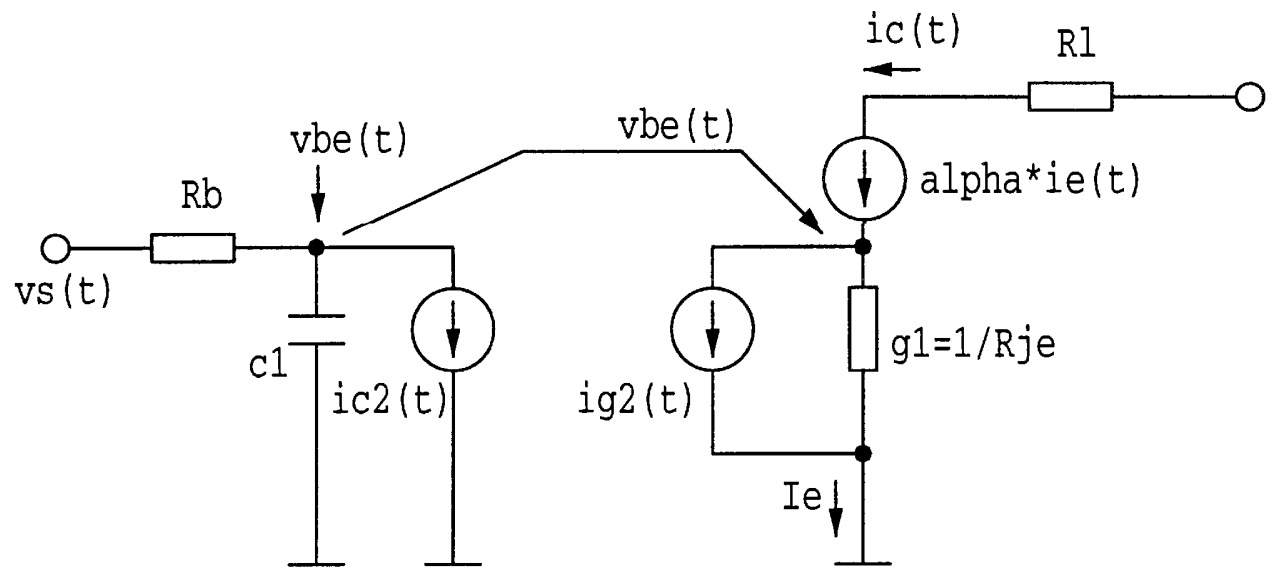

Figure 4.4: Generation and transfer of the nonlinear B-E voltage $v_{b e}$.

base resistance $R_{b}$ and the nonlinear base emitter capacitance ${ }^{2} C_{b e}$. This nonlinear circuit produces a voltage drop $v_{b e}$ across $C_{b e}$. Simulating ${ }^{3}$ this circuit as shown in Fig. 4.6 and considering only three harmonics, $v_{b e}$ has the following form:

$$
v_{b e}=V_{a} \cos \left(\omega t+\phi_{1}\right)+V_{b} \cos \left(2 \omega t+\phi_{2}\right)+V_{c} \cos \left(3 \omega t+\phi_{3}\right)
$$

Fig. 4.7 shows the Fourier frequency spectrum of $v_{b e}$. Fig. 4.8 shows the associated time waveform ${ }^{4}$. The magnitude of the input voltage source has been chosen very

\footnotetext{
${ }^{2}$ How $C_{b e}$ is represented by $C_{1}, I_{c, 2}$ and $I_{c, 3}$ is illustrated in section 4.7, i.e. $I_{c, 2}=\tilde{c}_{2} \frac{d\left(v^{2}\right)}{d t}$ and $I_{c, 3}=\tilde{c}_{3} \frac{d\left(v^{3}\right)}{d t}$.

${ }^{3} C_{b e}$ has been split into its linear, quadratic and cubic part, otherwise the implementation is as shown in sections 4.7 and 5.3.2.

${ }^{4}$ See appendix $G$ for detailed information on how to create the waveforms for the harmonics
} 


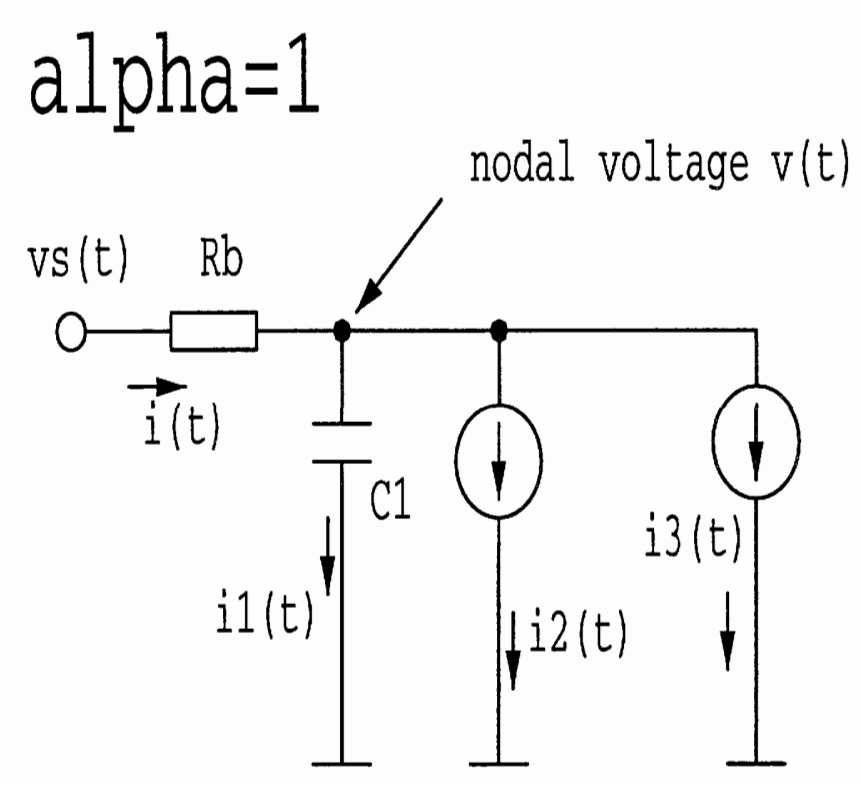

Figure 4.5: If $\alpha=1$ this circuit is decoupled from the circuit shown in Fig. 4.3. This is necessary to explore separately the nonlinear voltage drop which will drive the nonlinear currents through the conductance.

large so that the nonlinear effect, i.e. the difference (distortion) between the full frequency waveform and the fundamental, can be graphically shown. If the magnitude is smaller this distortion is hard to recognize. The crucial feature of this analysis is that the spectral line at the fundamental frequency has a phase $\phi_{1}$, see eq. 4.1 , which, when doubled is almost $180^{\circ}$ out of phase with the phase $\phi_{2}$ of the spectral line representing the second harmonic. Doubling the phase stems from the fact that the second order current source $i_{g 2}$ is caused by $\left(v_{b e}\right)^{2}$. In this specific example: $2 \phi_{1}=2 \cdot\left(-67.062^{\circ}\right)=-134.124^{\circ}$ is $185.666^{\circ}$ out of phase with $\phi_{2}=51.542^{\circ}$. and more. 


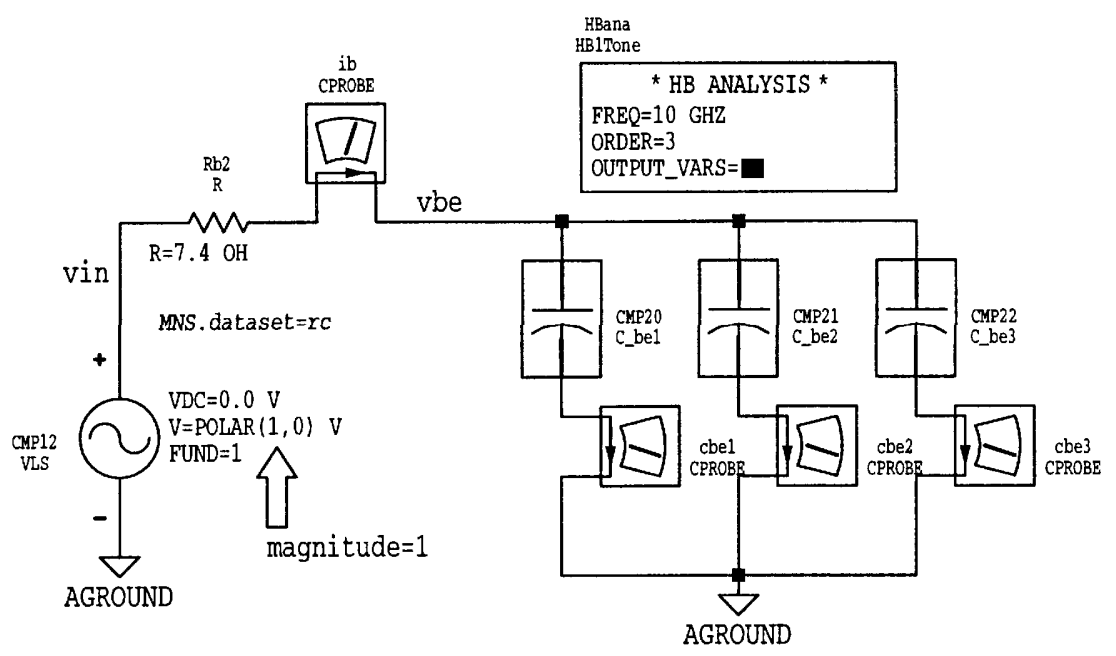

Figure 4.6: Simulation setup for the RC input circuit.

This represents the cancellation ${ }^{5}$ first discussed by Maas et al. [6]. Following the assumption that $\alpha=1, v_{b e}$ is also the voltage across the nonlinear base emitter conductance. Thus eq. 4.1 needs to be substituted into ${ }^{6}$

$$
i_{e}=g_{1} v_{b e}+g_{2} v_{b e}^{2}+g_{3} v_{b e}^{3}+\cdots,
$$

${ }^{5}$ Interestingly, the phase difference approaches $180^{\circ}$ if the magnitude of the input voltage is being increased, e.g. at $11 \mathrm{~V}$ the difference is $180.839^{\circ}$. Such a large input signal is impractical for the investigated HBT, however. This issue will be addressed in 4.9.1.

${ }^{6} \mathrm{How}$ a nonlinear conductance is represented and where eq. 4.2 comes from is shown in sections 4.6 and 5.3.1. 


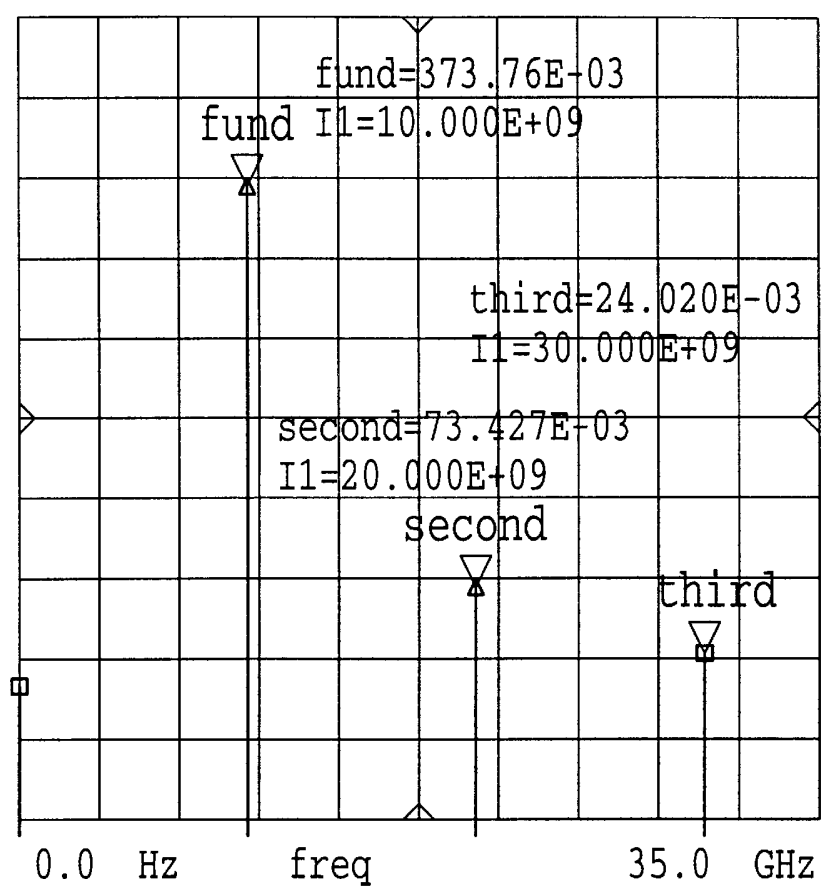

Figure 4.7: Spectral lines at the fundamental $(10 \mathrm{GHz})$, the second harmonic (20 $\mathrm{GHz})$ and the third harmonic $(30 \mathrm{GHz})$ of $V_{b e}$. The marker values denote the peak amplitude value of the associated waveform.

which gives ${ }^{7}$ :

$$
\begin{aligned}
i_{g}= & g_{1}\left[V_{a} \cos \left(\omega t+\phi_{1}\right)+V_{b} \cos \left(2 \omega t+\phi_{2}\right)+V_{c} \cos \left(3 \omega t+\phi_{3}\right)\right] \\
& +g_{2}\left[V_{a} \cos \left(\omega t+\phi_{1}\right)+V_{b} \cos \left(2 \omega t+\phi_{2}\right)+V_{c} \cos \left(3 \omega t+\phi_{3}\right)\right]^{2} \\
& +g_{3}\left[V_{a} \cos \left(\omega t+\phi_{1}\right)+V_{b} \cos \left(2 \omega t+\phi_{2}\right)+V_{c} \cos \left(3 \omega t+\phi_{3}\right)\right]^{3} .
\end{aligned}
$$

${ }^{7}$ The complete solution to eq. 4.3 has been computed with MAPLE and is shown in appendix B. How the nonlinear base emitter conductance is represented by $R_{j e}, I_{g, 2}$ and $I_{g, 3}$ is illustrated in appendix 4.6, i.e. $R_{j e}=1 / g_{1}$. The relation between $I_{g, 2} \Leftrightarrow g_{2} v^{2}$ and $I_{g, 3} \Leftrightarrow g_{3} v^{3}$ depends on how one decides to group the terms arising from eq. 4.3. Maas for example defines $I_{g, 2}=$ $0.5 g_{2} V_{a}^{2} \cos \left(2 \omega t+2 \phi_{1}\right)$ which presents a linear current source for the second harmonic. 


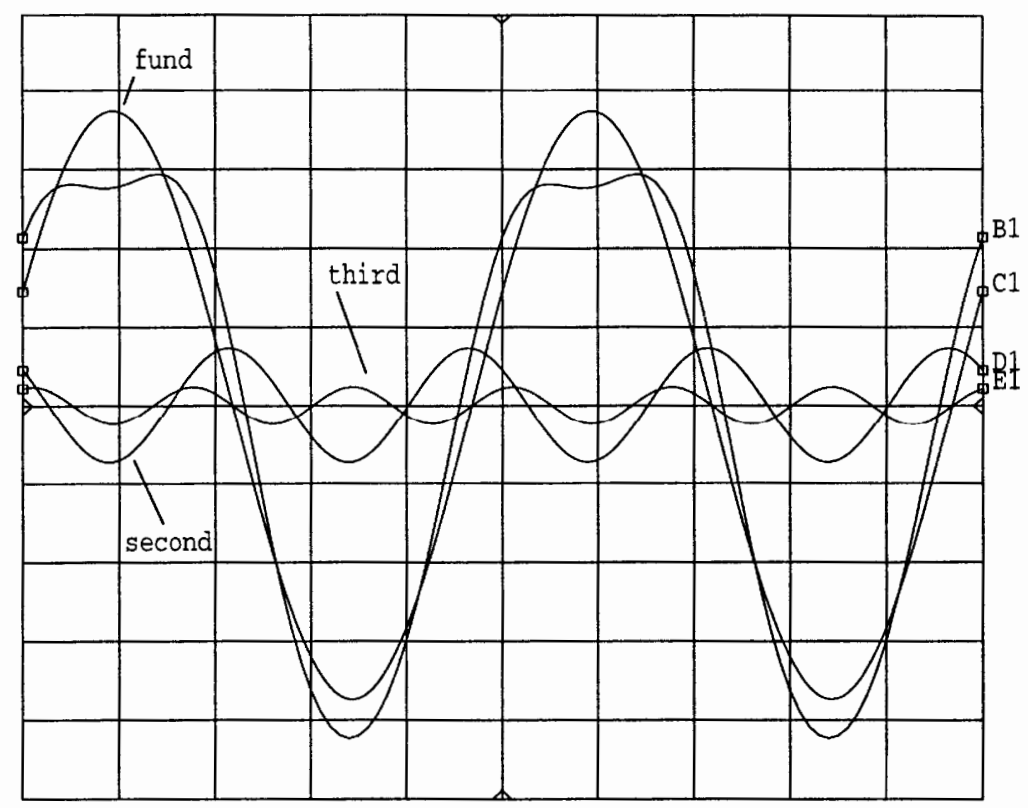

Figure 4.8: Time waveform of the spectral lines in Fig. 4.7

Because of their magnitude there are two terms ${ }^{8}$ in eq. 4.3 mainly responsible for the total emitter current at the second harmonic.

$$
i_{g, 2}(t)=\frac{1}{2} g_{2} V_{a}^{2} \cos \left(2 \omega t+2 \phi_{1}\right)+g_{1} V_{b} \cos \left(2 \omega t+\phi_{2}\right)
$$

Note that eq. 4.4 is the crux of the second order nonlinear current cancellation. While keeping the original assumption $(\alpha=1)$, Maas claims that the second order current components arising in the resistive and capacitive parts of the base emitter junction cancel each other, which implies that $2 \phi_{1}$ is $180^{\circ}$ out of phase with respect to

\footnotetext{
${ }^{8}$ There is a small conceptual problem here. The two terms are the leading terms only if the nonlinearity itself or its excitation is weak. Since for graphical illustration purposes the excitation was very strong in this example, i.e. 1 Volt, these terms are no longer the leading terms because higher harmonics enter the picture. Also, the generated dc component is very strong. Thus, the reasoning here gets back to the usual requirement that the nonlinearity or its excitation be mild.
} 
$\phi_{2}$. The explicit mentioning of capacitive and resistive parts in [6] may be misleading with respect to cancellation. The second order current cancellation, which reduces the second order distortion, takes place in the resistive part of the junction. But the voltage which causes the $180^{\circ}$ difference, which is the origin of this cancellation, is being developed in the capacitive part.

To present another argument for the cancellation consider the first order nonlinear differential eq. which governs the the $\mathrm{RC}$ input circuit.

$$
\frac{v_{s}}{R_{b}}=\frac{v_{b e}}{R_{b}}+c_{1} \frac{d v_{b e}}{d t}+c_{2} \frac{d v_{b e}^{2}}{d t}+c_{3} \frac{d v_{b e}^{3}}{d t}
$$

If $v_{s}=\cos (\omega t)$ then the simulation suggests ${ }^{9}$ that $v_{b e}=V_{b e, n} \cos \left(n \cdot \omega t+\phi_{n}\right)$ where the nonlinearity creates $\mathrm{n}$ possible harmonics. The boundary condition is introduced by $v_{s}$. If there were no resistance or other element causing a voltage drop, the only way to satisfy the boundary condition would be the non-existence of any frequency but the fundamental. Because $R_{b}$ introduces a new degree of freedom to satisfy the boundary condition, voltages at frequencies other than the fundamental exist. If $\mathrm{n}=2$ (i.e., the second harmonic) all ${ }^{10}$ second order components must add up to zero in order to satisfy eq. 4.5 since there is no second harmonic on the left hand side of eq. 4.5 .

\footnotetext{
${ }^{9}$ This agrees also with the well known fact that a harmonic input must result in a harmonic output if the nonlinearity is weak.

10 "All" alludes to the fact that here are more terms than the right hand side of eq. 4.4 proposes, which is indeed the case. But since weak nonlinearities or excitation are assumed, the two terms in eq. 4.4 are the leading terms.
} 


\subsection{Nonlinear Current Method}

The RC input circuit in Fig. 4.3 is revisited to illustrate the application of the nonlinear current method $^{11}$. Fig. 4.3 indicates that the nonlinear charge voltage relationship

$$
q(t)=c_{1} v(t)+c_{2} v^{2}(t)+c_{3} v^{3}(t)+\cdots
$$

describing the nonlinear base emitter capacitance $C_{b e}$, has been truncated after the cubic term. This implies a limitation to the third degree of the nonlinear relationship, where $q(t)$ and $v(t)$ are small signal quantities. If the nonlinearity or its excitation is weak, the following voltage relations based on the substitution theorem are the point of departure for the nonlinear current method. The voltage relations are limitated to the third order mixing products.

$$
\begin{aligned}
& v(t)=v_{1}(t)+v_{2}(t)+v_{3}(t) \\
& v^{2}(t)=v_{1}^{2}(t)+2 v_{1}(t) v_{2}(t) \\
& v^{3}(t)=v_{1}^{3}(t)
\end{aligned}
$$

The small signal nodal voltage $v(t)$ is indicated in Fig. 4.3. Because this voltage is nonlinear it contains many different frequencies. For example, $v_{1}(t)$ is the voltage at the fundamental and $v_{2}(t)$ is the voltage at the second harmonic. The corresponding counterparts in the frequency domain $V_{1}(\omega)$ and $V_{2}(2 \omega)$ are called first and second order voltages. A similiar nomenclature is used for the current components. Each

\footnotetext{
${ }^{11} \mathrm{~A}$ very good introduction to this method may be found in [14].
} 
frequency contains different voltage components. For example, there are many voltage components at the fundamental contributing to $v_{1}(t)$. They differ in magnitude and many of them can be neglected because their magnitude is very small.

The second term in eq. 4.8 presents a third order mixing product. The voltage relations in eq. 4.8 and eq. 4.9 can be assigned to the current sources in Fig. 4.3 as follows:

$$
\begin{aligned}
& i_{c 1}(t)=c_{1} \frac{d v_{1}(t)}{d t}+c_{1} \frac{d v_{2}(t)}{d t}+c_{1} \frac{d v_{3}(t)}{d t} \\
& i_{c 2}(t)=c_{2} \frac{d v_{1}^{2}(t)}{d t} \\
& i_{c 3}(t)=c_{2} \frac{d}{d t}\left[2 v_{1}(t) v_{2}(t)\right]+c_{3} \frac{d v_{1}^{3}(t)}{d t}
\end{aligned}
$$

The current sources in Fig. 4.3 represent all second- and third-order current components arising from eq. 4.6. The linear part of eq. 4.6, expressed by eq. 4.10, accounts for all other first- and higher-order current components flowing through the linear portion of $C_{b e}$, i.e. those currents that are proportional to $\frac{d v_{i}}{d t}$ where $v_{i}$ is any current component from eq. 4.10. It will turn out that $c_{1} \frac{d v_{2}(t)}{d t}$ and $c_{1} \frac{d v_{3}(t)}{d t}$ contribute little to the current through the linear portion of $C_{b e}$ so that $i_{c 1}(t)$ can be approximated by:

$$
i_{1}(t)=c_{1} \frac{d v_{1}(t)}{d t}
$$

This reshuffle entails two facts:

1. The circuit in Fig. 4.3 is linear, although the current sources are nonlinear functions of the voltage components of various order. 
2. The first order voltage $v_{1}(t)$ is generated by the first order source $v_{s}(t)$. The second order current is a function of the first order voltage. The third order current is a function of the first and second order voltages. Generally, the current of each order greater than one are functions of lower order voltages.

These two facts suggest a method of solution, which constitutes the nonlinear current method. The method basically consists of three steps:

1. Setting all current sources to zero, the first order voltage $v_{1}(t)$ is found under $v_{s}(t)$ excitation by a linear analysis.

2. The second order current source is then calculated by eq. 4.11. Setting $v_{s}(t)$ to zero and $i_{c 2}(t)$ the only excitation, the second order voltage $v_{2}(t)$ is found by a linear analysis at the second harmonic frequency.

3. The third order current source is given by eq. 4.12 . With $v_{s}(t)$ and $i_{c 2}(t)$ equal to zero and $i_{c 3}(t)$ the only excitation, the third order voltage follows from a linear analysis at the third harmonic frequency.

Because the circuit is linear, the total response, i.e. $v(t)$, of the circuit is a superposition of the responses to each individual excitation source. This means that in place of the nonlinear differential equation (eq. 4.5) for the total response, an associated set of linear differential equations for the components of the nonlinear response has been solved. The appropriate excitation at each stage is a function of the solutions for the lower order components of the nonlinear response obtained 
at a previous stage. In fact, these functions are the nonlinear transfer functions connecting the set of linear differential equations. The sum of the components is the series expansion of the total response.

\subsection{Taylor Series Expansion of a Nonlinear Con- ductance}

The base emitter junction may be represented by the usual diode equation.

$$
I_{e}=I_{0}\left(e^{\delta V_{b e}}-1\right) ; \delta=\frac{q}{\eta k T}
$$

Under small signal conditions this may be expanded into a Taylor series in the vicinity of the bias point ${ }^{12}$.

$$
i_{e}=\frac{\partial I_{e}}{\partial V_{b e}} v+\frac{1}{2} \frac{\partial^{2} I_{e}}{\partial V_{b e}^{2}} v^{2}+\frac{1}{6} \frac{\partial^{3} I_{e}}{\partial V_{b e}^{3}} v^{3}+\cdots=g_{1} v+g_{2} v^{2}+g_{3} v^{3}+\cdots
$$

The small signal currents and voltages are, of course, time dependent which has been omitted for convenience. It is assumed that $V_{b e} \gg v(t)$, assuring no runaway of the dc bias point. In addition, the nonlinearity is weak, assuring convergence of the series.

\footnotetext{
${ }^{12}$ How this is done in MAPLE is shown in appendix D.
} 


\subsection{Taylor Series Expansion of a Nonlinear Ca-}

\section{pacitance}

A nonlinear voltage controlled capacitor is defined in terms of its charge voltage relationship.

$$
Q_{b e}\left(V_{b e}\right)=\tau I_{e}=\tau I_{0}\left(e^{\delta V_{b e}}-1\right)
$$

Under small signal conditions this may be expanded into a Taylor series in the vicinity of the bias point.

$$
q_{b e}=\frac{\partial Q_{b e}}{\partial V_{b e}} v+\frac{1}{2} \frac{\partial^{2} Q_{b e}}{\partial V_{b e}^{2}} v^{2}+\frac{1}{6} \frac{\partial^{3} Q_{b e}}{\partial V_{b e}^{3}} v^{3}+\cdots=c_{1} v+c_{2} v^{2}+c_{3} v^{3}+\cdots
$$

In the linear case we would have $q_{b e}=C v$ which, when differentiated with respect to time, yields $i=C \frac{d v}{d t}$. In the nonlinear case it becomes:

$$
i=\frac{d q_{b e}}{d t}=\frac{d}{d t}\left(\frac{\partial Q_{b e}}{\partial V_{b e}} v+\frac{1}{2} \frac{\partial^{2} Q_{b e}}{\partial V_{b e}^{2}} v^{2}+\frac{1}{6} \frac{\partial^{3} Q_{b e}}{\partial V_{b e}^{3}} v^{3}+\cdots\right)
$$

Since $\frac{\partial Q_{b e}}{\partial V_{b e}}$ does not depend on time, and considering the chain rule for $v(t)$, it follows:

$$
i=\tilde{c}_{1} \frac{d v}{d t}+\tilde{c}_{2} \frac{d\left(v^{2}\right)}{d t}+\tilde{c}_{3} \frac{d\left(v^{3}\right)}{d t}
$$

The different notation, for example the use of tilde for the coefficients in eq. 4.19 with respect to 4.17 , reflects the fact the factors $\frac{1}{2}$ and $\frac{1}{6}$ will change due to the differentiation. 


\subsection{Introducing the Cancellation Term}

This cancellation term was first suggested by Maas et al. [6]. Because this term will occur in the following analytical derivations it will be introduced here. The coefficients $c_{1}, c_{2}, g_{1}, g_{2}$ follow from the Taylor series expansion of the large signal nonlinear relationships. For detailed information the reader is referred to appendix 5, D and $\mathrm{E}$ where the large signal relationships are expressed by Taylor series expansion using the program MAPLE.

$$
\begin{aligned}
& c_{1}=\tau \delta I_{0} e^{\delta V_{b e}} \\
& c_{2}=\frac{1}{2} \tau \delta^{2} I_{0} e^{\delta V_{b e}} \\
& g_{1}=\delta I_{0} e^{\delta V_{b e}} \\
& g_{2}=\frac{1}{2} \delta^{2} I_{0} e^{\delta V_{b e}}
\end{aligned}
$$

The cancellation term is formed by arranging the coefficients like:

$$
c_{2} g_{1}-c_{1} g_{2}=0
$$

It is somewhat difficult to assess the physical significance of this term following from a mere mathematical fact. Nevertheless, this term will prove useful on a couple of occasions. 


\subsection{Case 1 - Solving the RC Input Circuit by means of the Nonlinear Current Method}

The circuit solved by the nonlinear current method is shown in Fig. 4.3. The nonlinear current method starts by applying the excitation voltage of the form $v_{s}(t)=V_{s} \cos \omega t$. The first order voltage component $v_{1}(t)$ is found by a linear analysis when all current sources are set to zero and $v_{s}(t)$ is applied. Because the final goal is to find the phase relation between the first and second order voltage, no back conversion from the frequency domain to the time domain is needed. The first order voltage phasor $V_{1}(\omega)$ will therefore already suffice for our purposes because this notation displays explicitly the phase. Some of the MDS simultion results are, however, shown in the time domain for better illustration. In the following derivation a capital letter with explicit frequency dependence denotes a phasor, e.g. $Z_{1}(\omega)$, and a capital alone denotes its amplitude, e.g. $Z_{1}$. The complex impedance of the linear $\mathrm{RC}$ input circuit in the frequency domain is:

$$
Z_{1}(\omega)=R_{b}+\frac{1}{j \omega c_{1}}=\frac{\sqrt{\left(\omega c_{1} R_{b}\right)^{2}+1}}{\omega c_{1}} e^{-j \arctan \frac{1}{\omega c_{1} R_{b}}}
$$

The linear current in the frequency domain is:

$$
I_{1}(\omega)=\frac{V_{s}}{Z_{1}(\omega)}=\frac{\omega c_{1} V_{s}}{\sqrt{\left(\omega c_{1} R_{b}\right)^{2}+1}} e^{j \arctan \frac{1}{\omega c_{1} R_{b}}}
$$

and the voltage is:

$$
V_{1}(\omega)=\frac{I_{1}(\omega)}{j \omega c_{1}}=-j \frac{V_{s}}{\sqrt{\left(\omega c_{1} R_{b}\right)^{2}+1}} e^{j \arctan \frac{1}{\omega c_{1} R_{b}}}=V_{1} e^{j\left(\frac{3}{2} \pi+\arctan \frac{1}{\omega c_{1} R_{b}}\right)}
$$


where $-j=e^{\frac{3}{2} \pi}$ has been used. Because the phase of $V_{1}(\omega)$ will be of major concern it is repeated here:

$$
\angle V_{1}(\omega)=\phi_{1}=\frac{3}{2} \pi+\arctan \frac{1}{\omega c_{1} R_{b}} .
$$

To find the second order current component, eq. 4.11 is at first transformed into the frequency domain as follows: The phasor $V_{1}(\omega)$ converted into a time waveform gives $v_{1}(t)=V_{1} \cos \left(\omega t+\phi_{1}\right)$. Taking the square of $v_{1}(t)$ and neglecting the generated dc $\operatorname{term}^{13}$ gives $\frac{1}{2} V_{1}^{2} \cos \left(2 \omega t+2 \phi_{1}\right)$ which is subsituted into eq. 4.11 . When subsequently transforming eq. 4.11 remember that the derivative brings out a $j 2 \omega$, hence:

$$
I_{2}(2 \omega)=j 2 \omega c_{2} \frac{V_{1}^{2}(\omega)}{2}=j \omega c_{2} V_{1}^{2}(\omega)=j \omega c_{2} V_{1}^{2} e^{j\left(3 \pi+2 \arctan \frac{1}{\omega c_{1} R_{b}}\right)}
$$

and using $j=e^{\frac{1}{2} \pi}$ :

$$
I_{2}(2 \omega)=\omega c_{2} V_{1}^{2} e^{j\left(\frac{7}{2} \pi+2 \arctan \frac{1}{\omega c_{1} R_{b}}\right)}
$$

Now, with $I_{2}(2 \omega)$ as the only excitation $\left(v_{s}(t)\right.$ and the third order current source are set to zero) a linear analysis is performed at $2 \omega$ to find $V_{2}(2 \omega)$. The complex admittance for this circuit is:

$$
Y_{2}(2 \omega)=\frac{1}{R_{b}}+j 2 \omega c_{1}=\sqrt{\frac{1}{R_{b}^{2}}+\left(2 \omega c_{1}\right)^{2}} \cdot e^{j \arctan 2 \omega c_{1} R_{b}}
$$

and the second order voltage becomes:

$$
V_{2}(2 \omega)=-\frac{I_{2}(2 \omega)}{Y_{2}(2 \omega)}=\left|\frac{I_{2}(2 \omega)}{Y_{2}(2 \omega)}\right| e^{j\left(\frac{9}{2} \pi+2 \arctan \frac{1}{\omega c_{1} R_{b}}-\arctan 2 \omega c_{1} R_{b}\right)}
$$

\footnotetext{
${ }^{13}$ The important approximation of small nonlinearities comes into play again. Otherwise the dc term cannot be neglected.
} 
where $-1=e^{\pi}$ has already been accounted for. Because the phase of $V_{2}(2 \omega)$ will be of major concern it is repeated here:

$$
\angle V_{2}(2 \omega)=\phi_{2}=\frac{9}{2} \pi+2 \arctan \frac{1}{\omega c_{1} R_{b}}-\arctan 2 \omega c_{1} R_{b}
$$

\subsubsection{Cancellation in terms of Phase}

The cancellation mechanism has already been described in 4.4 and explicitly stated in eq. 4.4. Now, a relation between $2 \phi_{1}$ and $\phi_{2}$ is developed using the findings of the previous section:

$$
2 \phi_{1}=3 \pi+2 \arctan \frac{1}{\omega c_{1} R_{b}}
$$

and

$$
\phi_{2}=\frac{9}{2} \pi+2 \arctan \frac{1}{\omega c_{1} R_{b}}-\arctan 2 \omega c_{1} R_{b}
$$

If the phase difference between $V_{2}(2 \omega)$ and $V_{1}(\omega)$ is defined as $\phi_{2}-2 \phi_{1}$, one obtains the expression:

$$
\phi_{d i f f}=\frac{3}{2} \pi-\arctan 2 \omega c_{1} R_{b}
$$

which illustrates that $\phi_{\text {diff }}=\frac{3}{2} \pi$ if $\arctan 2 \omega c_{1} R_{b}=0$ and that $\phi_{\text {diff }}=\pi$ (i.e. the perfect cancellation in terms of phase) if $\arctan 2 \omega c_{1} R_{b} \rightarrow \frac{\pi}{2}$, which happens when $2 \omega c_{1} R_{b} \rightarrow \infty$. These observations are illustrated in Fig.4.9. Some further appreciation of eqs. 4.34 and 4.35 may be gained by finding a pattern which will show up in the cases explored later.

1. $\angle V_{1}(\omega)$ contains a term: 


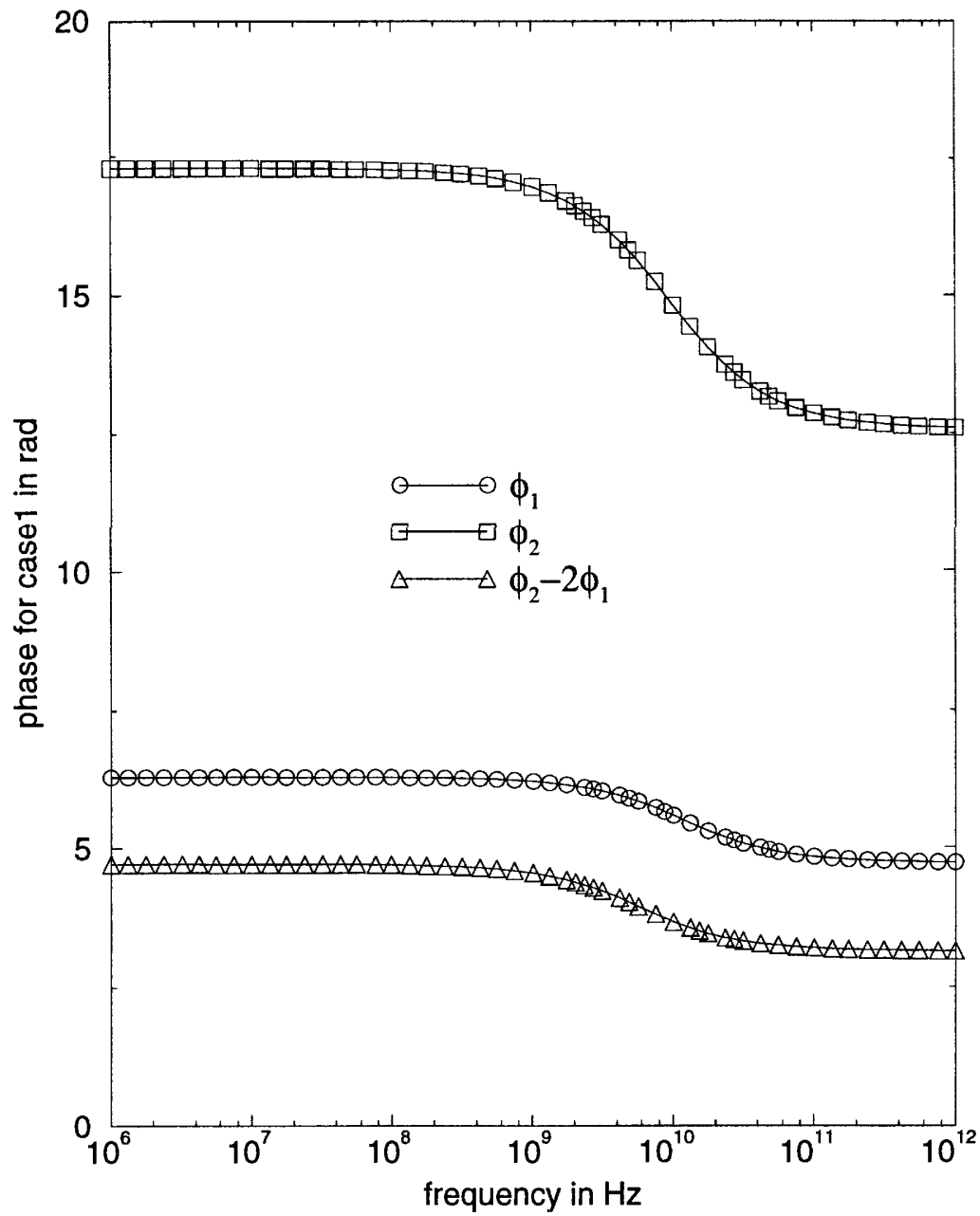

Figure 4.9: Phase variation with frequency for case 1

- $\arctan \frac{1}{\omega \mathrm{c}_{1} R_{b}}$.

2. $\angle V_{2}(2 \omega)$ contains, among other terms, exactly two times the above term of $\angle V_{1}(\omega)$, i.e.:

- $2 \arctan \frac{1}{\omega c_{1} R_{b}}$.

3. The argument of the above arctan function, i.e. $\frac{1}{\omega c_{1} R_{b}}$, occurs again at the second harmonic frequency. In this case it occurs inverted: 
- $\arctan 2 \omega c_{1} R_{b}$.

In other cases the same argument of the above arctan function occurs as is at the second harmonic frequency, e.g. in case 2, or the argument recurs inverted and noninverted as illustrated in case 4 .

Eq. 4.36 reflects also two of the limitations for cancellation that are mentioned in [6].

- If $\omega \rightarrow 0$ the cancellation is imperfect, i.e. it is $\frac{3}{2} \pi$. This suggests that if the excitation is a closely spaced two tone signal the cancellation at the second order intermodulation frequency $\omega_{1}-\omega_{2} \approx 0$ is insignificant.

- The term $2 \omega c_{1} R_{b}$ must be greater than unity for significant cancellation to occur. This term can be thought of as a corner frequency for an RC circuit. At the corner frequency the phase changes by $\frac{\pi}{4}$. As $\arctan 1=\frac{\pi}{4}$ this frequency may be found by $2 \omega c_{1} R_{b}=1$.

The impact of increased frequency is obvious in eq. 4.36 but there are more subtleties. It was mentioned in section 4.4 that an increased amplitude of the input signal leads to better cancellation. As the amplitude of the input signal increases the generated dc component becomes significant which is illustrated in appendix C. This in turn pushes up the bias point i.e. $V_{b e}$. The term $e^{V_{b e}}$ is the main contributor to the coefficients (and hence $c_{1}$ ) in the Taylor series expansion for the nonlinear capacitance $^{14}$.

\footnotetext{
${ }^{14}$ See appendix $\mathrm{D}$ for the taylor series expansion and 4.9.2 for the amplitude impact.
} 
Eq. 4.36 has been verified by numerical simulation (HB MDS) using the MAPLE computed coefficients $c_{1}, c_{2}, c_{3}$ and by analytical computation (MAPLE) ${ }^{15}$. To illustrate the verification, consider the following example: A simulation was run at $f=10$ $\mathrm{GHz}$, the input signal was $v_{s}(t)=1 m V \cos \omega t$, and 3 harmonics were considered. One simulation used fitted coefficients, the other the calculated coefficients. The fitted coefficients are from [6]. The coefficients were modified to match distortion results. The simulation which used the calculated Taylor coefficients was double checked by an analytical computation using MAPLE. The line marked by $\phi_{d i f f}^{*}$ calculated the phase difference using the phases from MDS or MAPLE right above the appropriate column. The line marked by $\phi_{\text {diff }}^{* *}$ used eq. 4.36 . The results are shown in Tab. 4.1 which prooves the validity of eq. 4.36. The difference between

\begin{tabular}{|l|l|l|r|}
\hline \hline & MDS, fitted coeff. & MDS, calc. coeff. & MAPLE, calc. coeff. \\
\hline$V_{1}(\omega)$ in $\mu V$ & $758.1 \angle-40.7^{\circ}$ & $762.3 \angle-40.3^{\circ}$ & $762.36 \angle-40.3^{\circ}$ \\
\hline$V_{2}(2 \omega)$ in $\mu V$ & $1.61 \angle 128.767^{\circ}$ & $4.467 \angle 129.846^{\circ}$ & $4.4687 \angle 129.845^{\circ}$ \\
\hline$\phi_{d i f f}^{*}$ & $210.17^{\circ}$ & $210.45^{\circ}$ & $210.45^{\circ}$ \\
\hline$\phi_{\text {diff }}^{* *}$ & $210.17^{\circ}$ & $210.51^{\circ}$ & $210.51^{\circ}$ \\
\hline \hline
\end{tabular}

Table 4.1: Comparison among two MDS simulations which used fitted and calculated taylor coefficients and the program MAPLE to test eq. 4.36.

the simultion results mainly at the second harmonic are due to the coefficients. MDS and MAPLE calculations agree perfectly when done using the same set of

\footnotetext{
${ }^{15}$ The executed MAPLE worksheet is given in appendix E. Note that MAPLE outputs arctan in rad and that the given example is for $\mathrm{f}=100 \mathrm{GHz}$.
} 
coefficients. Another simulation at another frequency $f=100 \mathrm{GHz}$ revealed the same perfect agreement between MDS and MAPLE.

\begin{tabular}{|l|l|l|r|}
\hline \hline & $c_{1}$ & $c_{2}$ & $c_{3}$ \\
\hline fitted coeff. & $1.85 \cdot 10^{-12}$ & $1.2 \cdot 10^{-11}$ & $4 \cdot 10^{-11}$ \\
\hline calc. coeff. & $1.8257 \cdot 10^{-12}$ & $3.2583 \cdot 10^{-11}$ & $38.767 \cdot 10^{-11}$ \\
\hline \hline
\end{tabular}

Table 4.2: Display of fitted and calculated coefficients

\subsubsection{Impact of Amplitude on Cancellation}

One of the surprising properties of nonlinearities is that the amplitude influences the phase. This has been alluded to in 4.4 by mentioning that an increased amplitude improves the cancellation. Improved cancellation means that $\arctan 2 \omega c_{1} R_{b} \rightarrow \frac{\pi}{2}$ which happens when $2 \omega c_{1} R_{b} \rightarrow \infty$. This section shows how an increased amplitude of the input signal increases the coefficient $c_{1}$. The example from the previous section is the starting point. A second MDS simulation was run with an input amplitude of $1 \mathrm{~V}$ as opposed to $1 \mathrm{mV}$. The following derivation shows how the resulting impact on the phase can be analytically accounted for.

The emitter current can be expanded ${ }^{16}$ in the vicinity of the bias point.

$$
i_{e}(t)=I_{0}\left(e^{\delta V_{b e}}-1\right)+I_{0} e^{\delta V_{b e}} \delta[v(t)]+\frac{1}{2} I_{0} e^{\delta V_{b e}} \delta^{2}[v(t)]^{2}
$$

\footnotetext{
${ }^{16}$ Shown in section 4.6 and appendix $\mathrm{D}$.
} 
where $\delta=\frac{q}{\eta k T}$. For this investigation, only the dc term and the second degree term are important. The small signal voltage $v(t)$ is, of course, sinusoidal and squaring it generates the dc term $\frac{1}{2} V^{2}$ where $\mathrm{V}$ is the amplitude.

$$
I_{e, \text { new }}=I_{0}\left(e^{\delta V_{b e}}-1\right)+\frac{1}{2} I_{0} e^{\delta V_{b e}} \delta^{2} \frac{V^{2}}{2}=I_{0}\left[e^{\delta V_{b e}}\left(1+\frac{1}{4} \delta^{2} V^{2}\right)-1\right]
$$

The factor $\delta V$ is unitless as it should be and since only dc terms are considered $i_{e}(t)$ becomes $I_{e, \text { new }}$. The subscript "new" means that this emitter current includes also the dc generated portion of the ac signal. The new emitter current offsets the bias voltage. Hence,

$$
I_{e, \text { new }}=I_{0}\left(e^{\delta V_{b e, \text { new }}}-1\right)
$$

Substituting eq. 4.38 into eq. 4.39 and cancelling $I_{0}$ gives:

$$
e^{\delta V_{b e}}\left(1+\frac{1}{4} \delta^{2} V^{2}\right)-1=e^{\delta V_{b e, \text { new }}}-1
$$

Solving this expression for $V_{b e, \text { new }}$ gives:

$$
V_{b e, \text { new }}=V_{b e}+\ln \left(1+\frac{1}{4} \delta^{2} V^{2}\right)
$$

$V_{\text {be,new }}$ occurs also in the Taylor series expansion of the nonlinear capacitance. The linear coefficient $c_{1}$ is of interest:

$$
c_{1, \text { new }}=\tau \delta I_{0} e^{\delta V_{\text {be, new }}}
$$

Substituting eq. 4.41 into eq. 4.42 gives:

$$
c_{1, \text { new }}=\underbrace{\tau \delta I_{0} e^{\delta V_{b e}}}_{c_{1}} e^{\ln \left(1+\frac{1}{4} \delta^{2} V^{2}\right)}=c_{1}\left(1+\frac{1}{4} \delta^{2} V^{2}\right) .
$$


The new value for $c_{1}$ from eq. 4.43 is plugged into eq. 4.36 and the results are compared with MDS simulation. As shown in Tab. 4.1, which displays the orginal simulation (i.e., the input amplitude is $1 \mathrm{mV}$ ), the phases of $V_{1}(\omega)$ and $V_{2}(2 \omega)$ are $\phi_{1}=-40.3^{\circ}$ and $\phi_{2}=129.846^{\circ}$, respectively. The phase difference between $V_{2}(2 \omega)$ and $\left(V_{1}(\omega)\right)^{2}$ is $210.446^{\circ}$. Employing eq. 4.36 to calculate the phase difference at an input amplitude of $1 \mathrm{mV}$ gives:

$$
\phi_{\text {diff }}=270^{\circ}-\arctan (2 \cdot \underbrace{2 \pi \cdot 10 \cdot 10^{9}}_{\omega} \cdot \underbrace{1.8257 \cdot 10^{-12}}_{c_{1}} \cdot \underbrace{7.4}_{R_{b}})=210.47^{\circ} .
$$

The simulation with excitation amplitude of $1 \mathrm{~V}$ gave $\angle V_{1}(\omega)=\phi_{1}=-78.683^{\circ}$ and $\angle V_{2}(2 \omega)=\phi_{2}=24.748^{\circ}$, respectively. The phase difference between $V_{2}(2 \omega)$ and $\left(V_{1}(\omega)\right)^{2}$ is then $182.114^{\circ}$. Employing eq. 4.43 with the new value for $c_{1}$ gives:

$$
c_{1, \text { new }}=c_{1}\left(1+\frac{1}{4} \cdot 35.694^{2} \cdot 1^{2}\right)=c_{1} \cdot 319.5
$$

and substituting eq. 4.45 into eq. 4.44 results in $\phi_{\text {diff }}=180.1^{\circ}$ which proves that the analytical method outlined here accounts for the influence of amplitude on phase. 


\subsection{Case 2}

The appropriate circuit in Fig.4.3 is now investigated. The assumption $\alpha=1$ is dropped. In fact $\alpha=0.9764$. The remitter resistance is still ignored. The circuit is redrawn in Fig.4.10 for convenience. The voltages $V_{1}(\omega)$ and $V_{2}(2 \omega)$ are

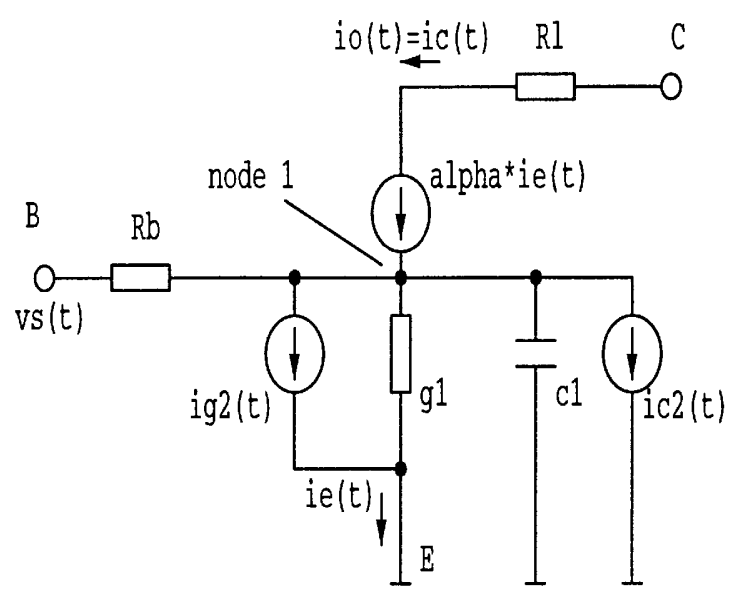

Figure 4.10: Circuit for case 2.

the nodal voltages at node 1 at the fundamental and second harmonic, respectively. Note that the emitter current is defined through the conductive portion of the base emitter junction only and that the nodal voltages equal the terminal voltages of the nonlinearities. $V_{1}(\omega)$ is described by:

$$
V_{1}(\omega)=\frac{V_{s}}{1+g_{1} R_{b}(1-\alpha)+j \omega c_{1} R_{b}} .
$$


$V_{2}(2 \omega)$ is described by:

$$
V_{2}(2 \omega)=-\frac{R_{b}\left[I_{g 2}(1-\alpha)+I_{c 2}\right]}{1+g_{1} R_{b}(1-\alpha)+j 2 \omega c_{1} R_{b}} .
$$

The phase of $V_{1}(\omega)$ is given as:

$$
\phi_{1}=-\arctan \frac{\omega c_{1} R_{b}}{g_{1} R_{b}(1-\alpha)+1}
$$

and of $V_{2}(2 \omega)$ as:

$$
\begin{aligned}
\phi_{2}= & \pi-2 \arctan \frac{\omega c_{1} R_{b}}{g_{1} R_{b}(1-\alpha)+1}-\arctan \frac{2 \omega c_{1} R_{b}}{g_{1} R_{b}(1-\alpha)+1} \\
& +\arctan \frac{2 \omega c_{2}}{g_{2}(1-\alpha)} .
\end{aligned}
$$

By comparing eqs. 4.48 and 4.49 the pattern mentioned in section 4.9 .1 can be observed, i.e., $\phi_{2}$ contains excatly a term twice the value of $\phi_{1}$ and the argument of this term recurs at the second harmonic frequency. The phase difference between $V_{2}(2 \omega)$ and $\left(V_{1}(\omega)\right)^{2}$ is given as:

$$
\phi_{\text {diff }}=\phi_{2}-2 \phi_{1}=\pi-\arctan \frac{2 \omega c_{1} R_{b}}{1+g_{1} R_{b}(1-\alpha)}+\arctan \frac{2 \omega c_{2}}{g_{2}(1-\alpha)},
$$

which simplifies to eq. 4.36 if $\alpha=1$. Eq. 4.50 may be rewritten to show the cancellation effect described in [6].

$$
\phi_{\text {diff }}=\pi+\arctan 2 \omega \frac{c_{2}+\left(c_{2} g_{1}-g_{2} c_{1}\right) R_{b}(1-\alpha)}{g_{2}(1-\alpha)\left[1+g_{1} R_{b}(1-\alpha)\right]+4 \omega^{2} c_{2} c_{1} R_{b}} .
$$

If the coefficients $g_{1}, g_{2}, c_{1}$ and $c_{2}$ are replaced by their values from the Taylor series expansion of their respective large signal nonlinear relationship, the term $c_{2} g_{1}-g_{2} c_{1}$ evaluates exactly to zero, reducing the argument of the arctan and bringing the phase difference closer to $\pi$. Both eq. 4.50 and eq. 4.51 suggest that higher frequencies 
result in better cancellation which is illustrated in Fig. 4.11 and Fig. 4.14. The importance of the term $c_{2} g_{1}-g_{2} c_{1}$ can be restated: It reduces the frequencies at which cancellation occurs. The cancellation term looses its strength if the base emitter capacitance is weakly nonlinear. Weakly nonlinear means small $c_{2}$. This was already suggested in [6]. Computing the results from eqs. 4.50 and 4.51 gives

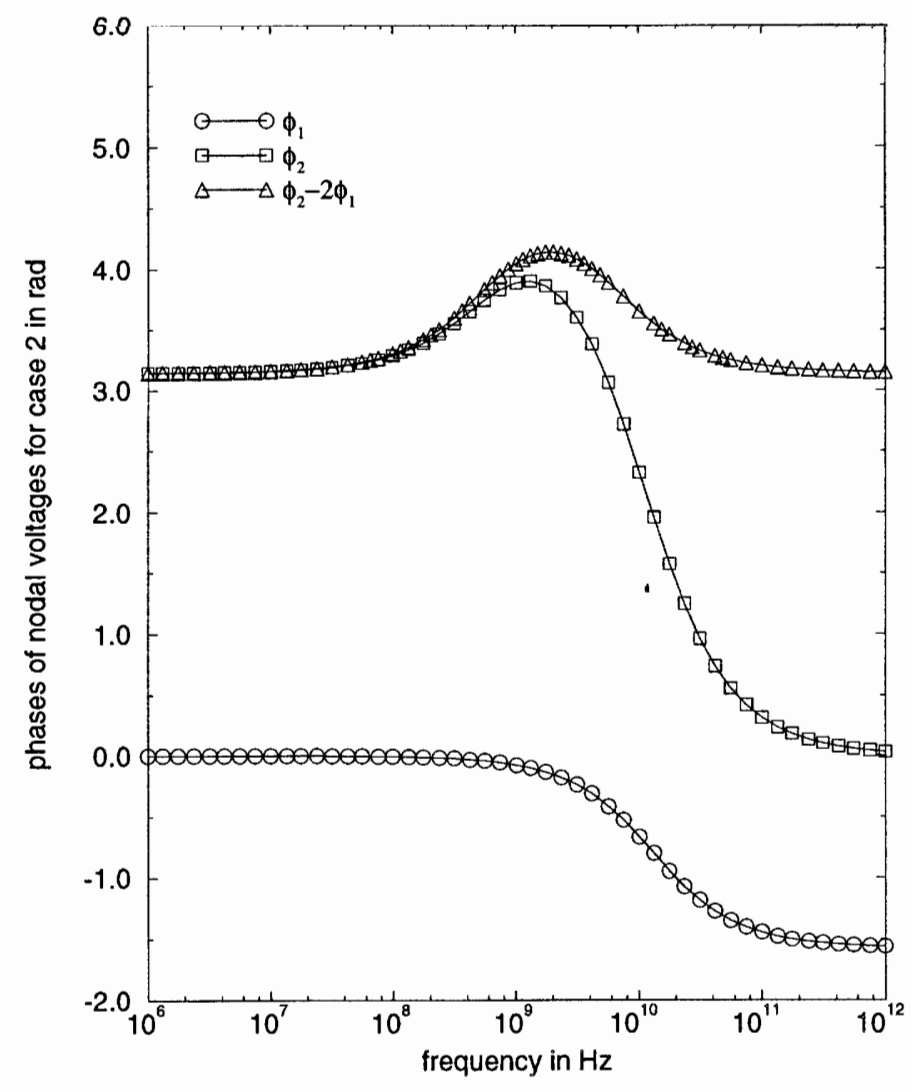

Figure 4.11: The overshoot of $\phi_{2}$ immediately before the fall-off is mainly responsible for the deviation of $\phi_{\text {diff }}$ from $\pi$.

$\phi_{\text {diff }}=209.6^{\circ}$ at $\mathrm{f}=10 \mathrm{GHz}$ which perfectly agrees with the MDS simulation result and also indicates the identity of eqs. 4.50 and 4.51 . 


\subsubsection{Current Cancellation}

The phase difference translates directly into the output current $I_{c}(2 \omega)=\alpha I_{e}(2 \omega)$ because the emitter current is assumed to be flowing only through the conductive portion of the junction. The time waveforms from MDS results at $f=10 \mathrm{GHz}$ are shown in Fig.4.12. The definition of emitter current automatically simulates the

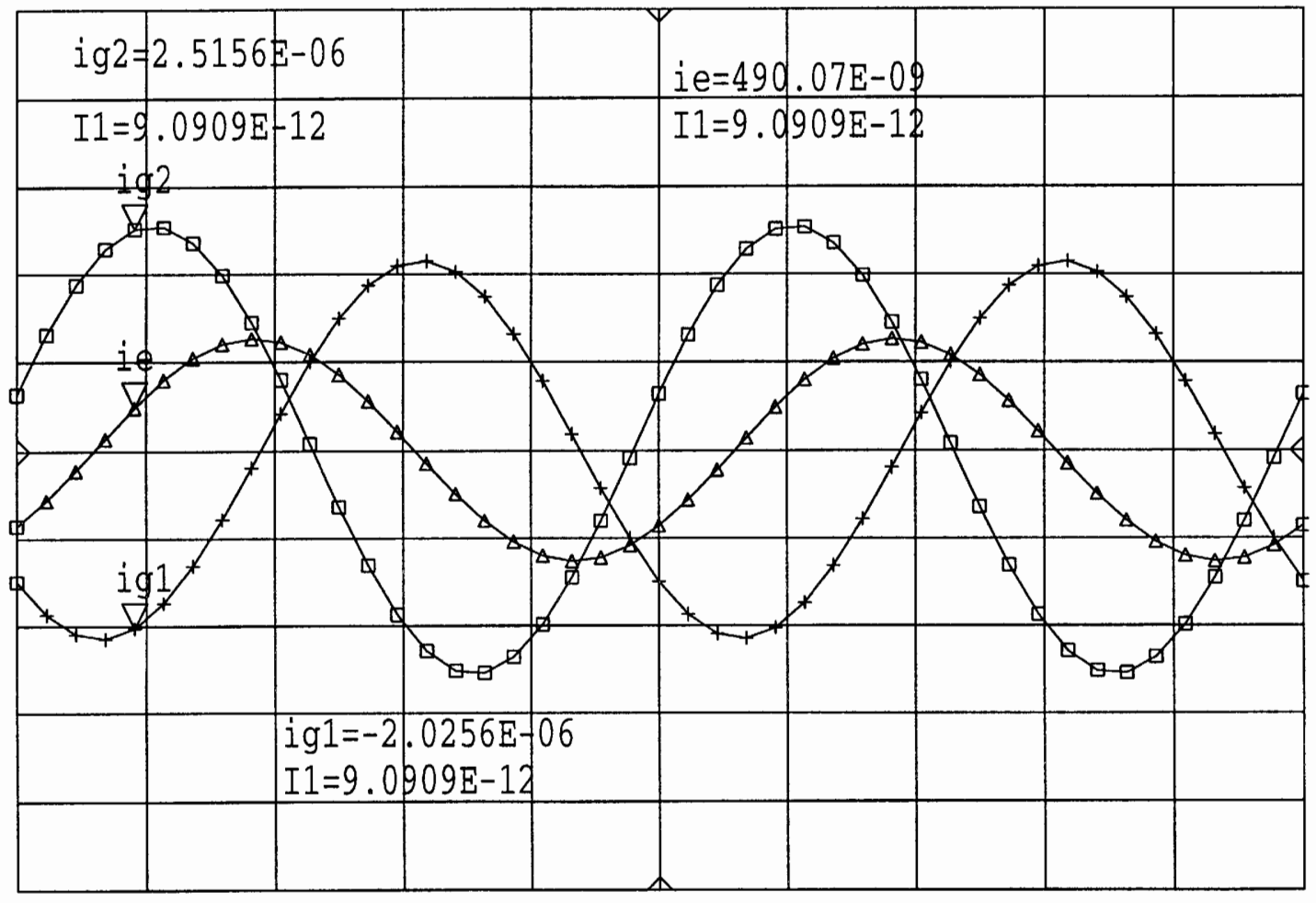

Figure 4.12: The marker ig1, ig2 and ie denote the time waveforms of the phasors $I_{g 1}(2 \omega), I_{g 2}(2 \omega)$ and $I_{e}(2 \omega)$ in the frequency domain, respectively and have been set at $\mathrm{t}=9.09$ ps. $I_{g 1}(2 \omega)$ and $I_{g 2}(2 \omega)$ are the currents through the linear $\left(g_{1}\right)$ and the nonlinear part $\left(g_{2}\right)$ of the nonlinear conductance $g$. $I_{e}(2 \omega)$ is the emitter current $\left(i_{g 1}(t)+i_{g 2} t\right)=i_{e}(t), \mathrm{f}=10 \mathrm{GHz}$, case 2$)$. 
gain roll-off at higher frequencies because more current goes through the capacitor as its impedance becomes smaller at higher frequencies, leaving simultaneously less current through the conductance, therefore less output current. Therefore no direct frequency dependence of $\alpha$ is needed. The resulting lower emitter current at high frequencies causes lower collector current which degrades the common emitter current gain $\beta=\frac{I_{c}}{I_{b}}$. The frequency where this fraction decreases by $\frac{1}{\sqrt{2}}$ is the corner frequency which was found to be $f_{c o, \beta}=1.13215 \mathrm{GHz}$ which coincides with the value $^{17}$ given in [6]. The phasor diagram associated with the time waveforms in

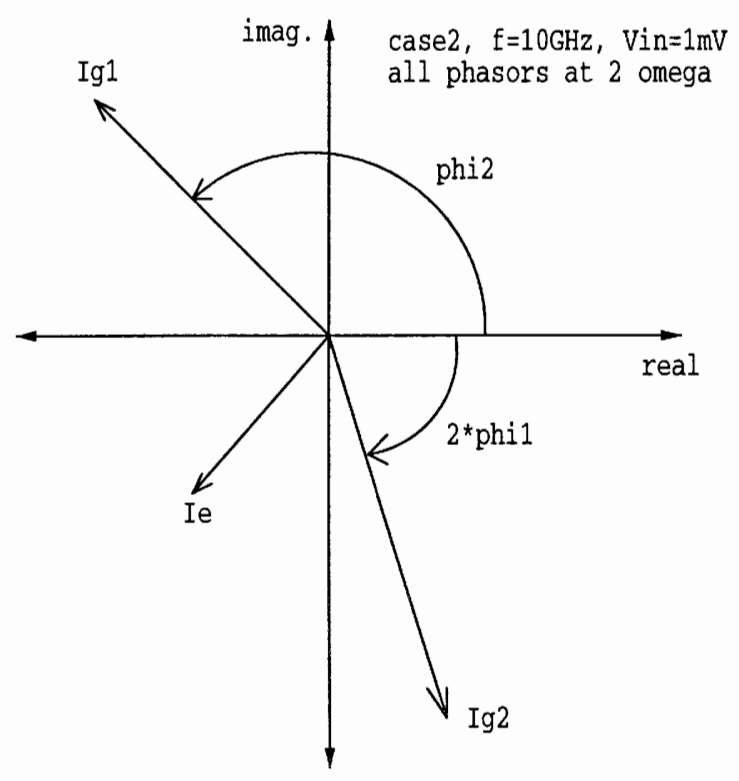

Figure 4.13: The corresponding phasor diagram to the time waveforms in Fig.4.12

\footnotetext{
${ }^{17}$ Maas [6] labels it as the cutoff frequency for cancellation effects.
} 
Fig.4.12 is shown in Fig. 4.13.

Another simulation was run to test if higher frequencies improve cancellation. The current timewaveforms for a simulation at $50 \mathrm{GHz}$ are shown in Fig.4.14. The

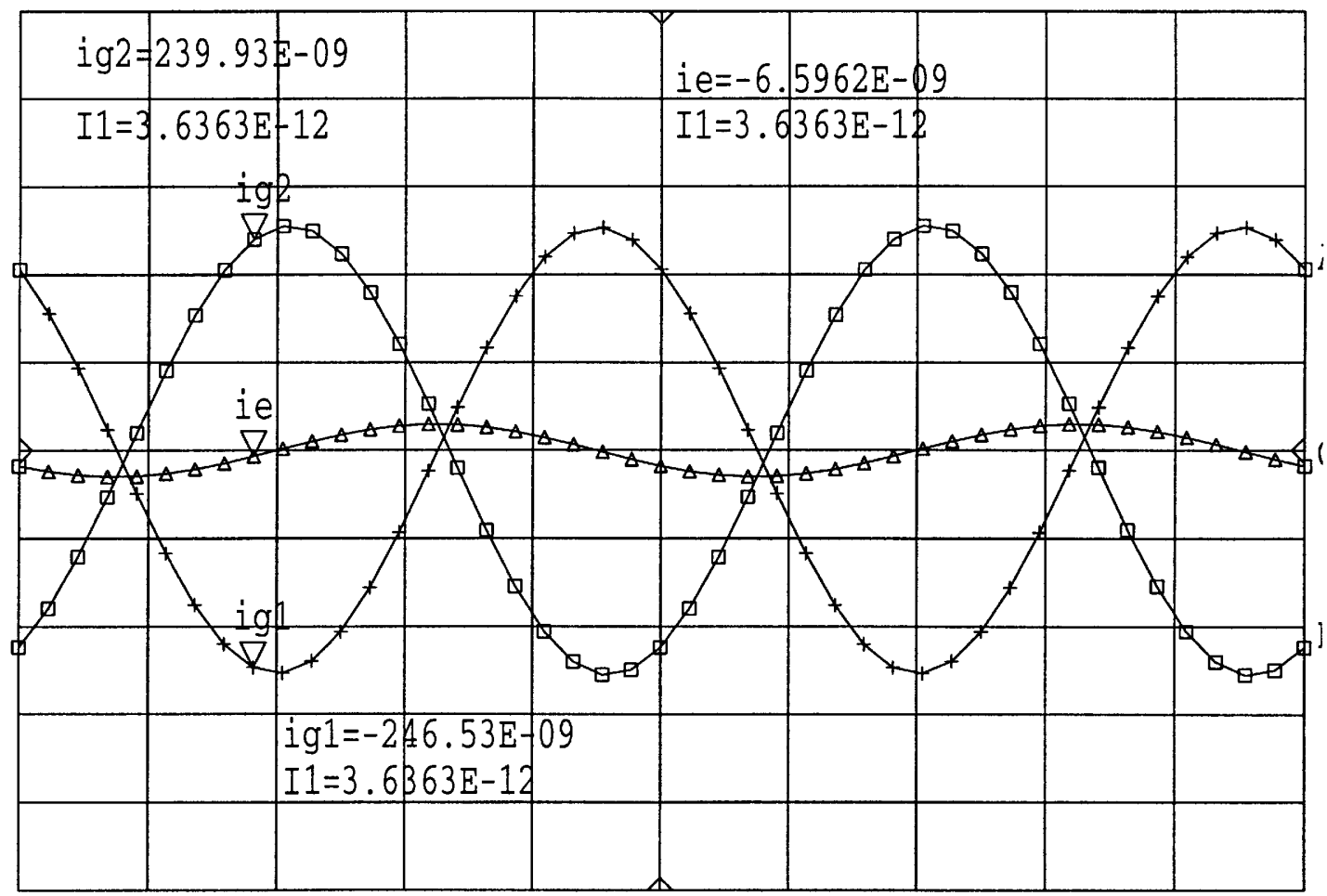

Figure 4.14: The reduced emitter current is due to better cancellation and due to its definition, namely that more current flows through the B-E capacitance at higher frequencies $\left(I_{g 1}(2 \omega)+I_{g 2}(2 \omega)=I_{e}(2 \omega), f=50 \mathrm{GHz}\right.$, case 2$)$.

greatly reduced emitter current in comparison to Fig. 4.12 causes better IP2 $=38.2$ $\mathrm{dBm}$ as opposed to $25.7 \mathrm{dBm}$ for $10 \mathrm{GHz}$. The reasoning goes like this:

- the closer the phase difference to $\pi$, 
- the better the second order current canncellation in the base emitter junction,

- the smaller the emitter current at the second harmonic,

- the smaller the output current $I_{c}(2 \omega)=I_{\text {out }}(2 \omega)=\alpha \cdot I_{e}(2 \omega)$,

- the smaller the output power at the second (undesired) harmonic $P_{\text {out }}(2 \omega)=$ $R_{l} \cdot I_{\text {out }}^{2}(2 \omega)$

- the lower the second order distortion IMD2,

- the higher the linearity of the device,

- the higher IP2.

Also, the nonlinear capacitance does not significantly affect the magnitude of the nonlinear currents. As a matter of fact, the magnitude of the currents flowing through the B-E capacitance are smaller by several orders of magnitude than the currents flowing through the B-E conductance. This has been double checked by comparing the output current to the emitter current and by looking at the currents steming from the B-E capacitance itself. This fact supports the conclusion that the current cancellation takes place among currents in the B-E conductance and that the importance of the B-E capacitance comes from its influence on the phase.

We will now turn to case 3 which, at first sight, may seem to be very different but it will become clear that case 3 is identical to case 2 . 


\subsection{Case 3}

The base emitter junction is now modeled as a parallel combination of nonlinear conductance and capacitance with the emitter current defined through both. This

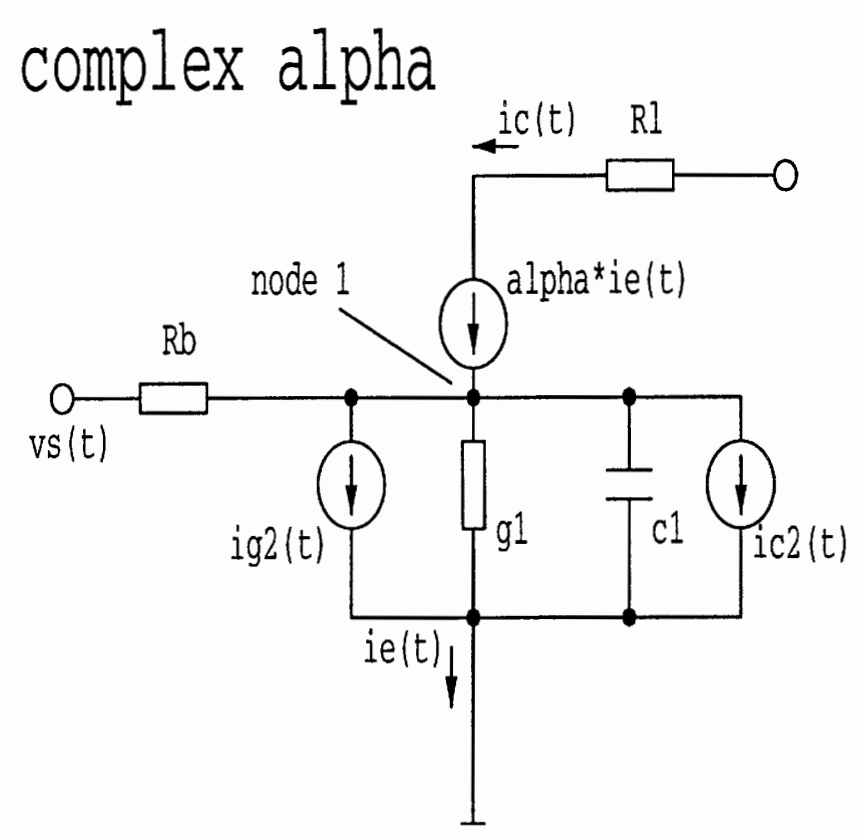

Figure 4.15: Equivalent circuit model for case 3. Note the definition of emitter current which entails a complex $\alpha$ as given in eq. 4.52 .

also implies $\alpha \neq 1$ but the gain roll-off has to be explicitely modeled in $\alpha$. The usual approach is:

$$
\alpha=\frac{\alpha_{0}}{1+j \frac{\omega}{\omega_{c o, a}}}
$$

where $\omega_{c o, \alpha}=2 \pi f_{c o, \alpha}$ indicates a corner frequency for $\alpha$ and $\alpha_{0}$ is the low frequency value of $\alpha$. As $\omega_{c o, \beta}=2 \pi f_{c o, \beta}$ is not necessarily equal to $\omega_{c o, \alpha}$ a relation between 
the two may be established as follows:

$$
\beta=\frac{\alpha}{1-\alpha}
$$

The value for $\omega_{c o, \beta}$ has been determined from case 2 by checking at which frequency value $\beta=\frac{I_{c}}{I_{b}}$ was reduced by a factor of $\frac{1}{\sqrt{2}}$. Substituting eq.4.52 into eq.4.53 gives:

$$
\beta=\frac{\alpha_{0}}{1-\alpha_{0}+j \frac{\omega \prime}{\omega_{c o, \alpha}}}
$$

For finding the relation between $\omega_{c o, \beta}$ and $\omega_{c o, \alpha}$ eq.4.54 is manipulated into the form of Eq.4.53.

$$
\beta=\frac{\frac{\alpha_{0}}{1-\alpha_{0}}}{1+j \frac{\omega}{\omega_{c,, \alpha}\left(1-\alpha_{0}\right)}} .
$$

Apparently, $f_{c o, \beta}=f_{c o, \alpha}\left(1-\alpha_{0}\right)$ and it follows:

$$
f_{c o, \alpha}=\frac{f_{c o, \beta}}{1-\alpha_{0}}=\frac{1.13215 G H z}{1-0.9764}=47.97 G H z .
$$

The time delay effect $I_{c}=\alpha I_{e}\left(t-t_{d}\right)$ could also be considered in Eq.4.52 by multiplying $\alpha_{0}$ with the term $e^{-j \omega t_{d}}$ which represents the corresponding Fourier factor. It is not included here to simplify the analytical derivation.

\subsubsection{Low Frequency}

Apparently, if $\omega \ll \omega_{\text {co }}$ then $\alpha \approx \alpha_{0}$. The following derivation assumes this. The phase of $V_{1}(\omega)$ is given as:

$$
\phi_{1}=\arctan \frac{\omega c_{1} R_{b}(\alpha-1)}{g_{1} R_{b}(1-\alpha)+1}
$$


and of $V_{2}(2 \omega)$ as:

$$
\begin{aligned}
\phi_{2}= & 2 \arctan \frac{\omega c_{1} R_{b}(\alpha-1)}{g_{1} R_{b}(1-\alpha)+1}+\arctan \frac{2 \omega c_{1} R_{b}(\alpha-1)}{1+g_{1} R_{b}(1-\alpha)} \\
& +\arctan \frac{2 \omega c_{2}}{g_{2}}-\pi
\end{aligned}
$$

By comparing eqs. 4.57 and 4.58 the pattern mentioned in section 4.9 .1 can be observed. The phase difference comes out to be:

$$
\phi_{\text {diff }}=-\arctan \frac{2 \omega c_{1} R_{b}(\alpha-1)}{1+g_{1} R_{b}(1-\alpha)}+\arctan \frac{2 \omega c_{2}}{g_{2}}-\pi .
$$

Eq. 4.59 may be rewritten to show the cancellation effect $\left(c_{2} g_{1}-g_{2} c_{1}=0\right)$ described in $[6]$.

$$
\phi_{\text {diff }}=\arctan 2 \omega \frac{c_{2}+\left(c_{2} g_{1}-g_{2} c_{1}\right) R_{b}(1-\alpha)}{g_{2}+R_{b}(1-\alpha)\left(4 \omega^{2} c_{2} c_{1}+g_{1} g_{2}\right)}-\pi .
$$

\subsubsection{Complex $\alpha$}

Different frequency ranges need to be distinguished. As will be shown later the border line for $\phi_{1}$ is:

$$
f=\sqrt{\frac{f_{c o, \alpha}\left[1+R_{b} g_{1}\left(1-\alpha_{0}\right)\right]}{2 \pi c_{1} R_{b}}}
$$

which follows from the denominator of the second term in eq. 4.68. Due to the corner frequency the denominator goes through zero causing a phase jump. This phase jump is somewhat artificial and it does not really matter as it is a jump of $2 \pi$. Nevertheless, the denominator in eq. 4.68 clearly indicates a singularity. To gain some insight in eq. 4.61 an expression for $f_{c o, \alpha}$ needs to be found. Since $\alpha$ in the model of case 2 is only a constant $\left(\alpha=\alpha_{0}\right)$ the frequency dependence is brought 
into play by $\beta$. In the linear case it follows from the nodal equation that at the fundamental: frequency

$$
\beta=\frac{I_{c}}{I_{b}}=\frac{\frac{\alpha_{0}}{1-\alpha_{0}}}{1+j \frac{\omega c_{1}}{g_{1}\left(1-\alpha_{0}\right)}}=\frac{\frac{\alpha_{0}}{1-\alpha_{0}}}{1+j \frac{\omega}{\omega_{c 0_{0}, \beta}}} .
$$

By inspection:

$$
\omega_{c o, \beta}=\frac{g_{1}\left(1-\alpha_{0}\right)}{c_{1}}
$$

It was found earlier that $\omega_{c o, \beta}=\omega_{c o, \alpha}\left(1-\alpha_{0}\right)$. Thus,

$$
f_{\mathrm{co}, \alpha}=\frac{g_{1}}{2 \pi c_{1}} .
$$

Multiplying the denominator under the square root in eq. 4.61 with $\frac{g_{1}}{g_{1}}$, using eq. 4.64 and neglecting the term in brackets, eq. 4.61 gives the critical frequency for $\phi_{1}$ and simplifies to:

$$
f_{c r 1}=f_{c o, \alpha} \frac{1}{\sqrt{g_{1} R_{b}}}
$$

In this specific case ${ }^{18}: g_{1}=0.55$ and $R_{b}=7.4$ so that $f_{c r} \approx \frac{f_{c o, \alpha}}{2} . V_{1}(\omega)$ is described by:

$$
V_{1}(\omega)=\frac{V_{s}}{1+\left(g_{1}+j \omega c_{1}\right) R_{b}\left(1-\frac{\alpha_{0}}{1+j \frac{\omega}{\omega \omega_{c o, \alpha}}}\right)} .
$$

If $f \leq f_{c r 1}$ the phase of $V_{1}(\omega)$ is given as:

$$
\phi_{1}=\arctan \frac{\omega}{\omega_{c o, \alpha}}+\arctan \frac{\omega\left[1+R_{b}\left(g_{1}+\omega_{c o, \alpha} c_{1}\left(1-\alpha_{0}\right)\right)\right]}{-\omega_{c o, \alpha}\left[1+R_{b} g_{1}\left(1-\alpha_{0}\right)\right]+\omega^{2} c_{1} R_{b}} .
$$

If $f>f_{c r 1}$ the phase of $V_{1}(\omega)$ is given as:

$$
\phi_{1}^{\prime}=\phi_{1}-\pi \text {. }
$$

\footnotetext{
${ }^{18}$ Too much importance should not be given to this expression as the relation of $g_{1}$ and $R_{b}$ is arbitrary.
} 
From a mathematical point of view the singularity is interesting. Evaluating eq. 4.68 for $f=f_{\text {cr } 1}=\frac{f_{c o, \alpha},}{2}$ leads to:

$$
\phi_{1}=\arctan \frac{1}{2}+\frac{\pi}{2}-\pi=-1.1 .
$$

For this example, $f_{c o, \alpha} \approx 48 \mathrm{GHz}$. Running MAPLE and MDS at $24 \mathrm{GHz}$ gives $-1.08 \mathrm{rad}$ for $\phi_{1}$.

Similiarly to $\phi_{1}$ there are singularities for $\phi_{2}$. There are three ranges for $\phi_{2}$ which follow from the denominators of the second and fifth term in eq. 4.72. The new constrain for $\phi 2$ is introduced by:

$$
f_{c r 2}=\sqrt{\frac{f_{c o, \alpha}\left[1+R_{b} g_{1}\left(1-\alpha_{0}\right)\right]}{4 \cdot 2 \pi c_{1} R_{b}}}=\frac{f_{c o, \alpha}}{2 \sqrt{g_{1} R_{b}}}
$$

where the last expression in eq. 4.70 follows from the same reasoning as above. In this specific case the critical phase for $\phi 2$ is at $f_{c r 2} \approx \frac{f_{c o, \alpha}}{4}$. If $f_{c r 2}<f \leq f_{c r 1}, \phi_{2}$ is given by:

$$
\begin{aligned}
\phi_{2}= & 2 \arctan \frac{\omega}{\omega_{c o, \alpha}}+2 \arctan \frac{\omega\left[1+R_{b}\left(g_{1}+\omega_{c o, \alpha} c_{1}\left(1-\alpha_{0}\right)\right)\right]}{-\omega_{c o, \alpha}\left[1+R_{b} g_{1}\left(1-\alpha_{0}\right)\right]+\omega^{2} c_{1} R_{b}} \\
& +\arctan \frac{2 \omega c_{2}}{g_{2}}+\arctan \frac{2 \omega}{\omega_{c o, \alpha}\left(1-\alpha_{0}\right)} \\
& +\arctan \frac{2 \omega\left[1+R_{b}\left(g_{1}+\omega_{c o, \alpha} c_{1}\left(1-\alpha_{0}\right)\right)\right]}{-\omega_{c o, \alpha}\left[1+R_{b} g_{1}\left(1-\alpha_{0}\right)\right]+4 \omega^{2} c_{1} R_{b}} .
\end{aligned}
$$

The phase difference for this range is also given by eq. 4.73. If $f>f_{c r 1}$ the phase of $V_{2}(2 \omega)$ is given as:

$$
\phi_{2}^{\prime \prime}=\phi_{2}-2 \pi
$$

and the phase difference as:

$$
\phi_{\text {diff }}=\arctan \frac{2 \omega}{\omega_{c o, \alpha}\left(1-\alpha_{0}\right)}+\arctan \frac{2 \omega c_{2}}{g_{2}}
$$




$$
+\arctan \frac{2 \omega\left[1^{\circ}+R_{b}\left(g_{1}+\omega_{c o, \alpha} c_{1}\left(1-\alpha_{0}\right)\right)\right]}{-\omega_{c o, \alpha}\left[1+R_{b} g_{1}\left(1-\alpha_{0}\right)\right]+4 \omega^{2} c_{1} R_{b}}
$$

If $f \leq f_{\text {cr } 2}$ the phase of $V_{2}(2 \omega)$ is given as:

$$
\phi_{2}^{\prime}=\phi_{2}+\pi
$$

and the phase difference as:

$$
\phi_{d i f f}^{\prime}=\pi+\phi_{d i f f}
$$

These equations give the same values as the corresponding expressions in 4.10 . A quick look at the limits of eqs. $4.73,4.68$ and 4.72 reveals the parallels as well: If $f \rightarrow \infty$ then $\phi_{\text {diff }} \rightarrow \pi, \phi_{1} \rightarrow-\frac{\pi}{2}$ and $\phi_{2} \rightarrow 0$. The expressions for $\phi_{2}$ hold for frequencies around the ranges given. Far away from these boundaries additional phase jumps may occur, e.g. at $f=16 \mathrm{MHz}$ the correct value for $\phi_{2}$ is given by eq. 4.71 minus $\pi$. The complex $\alpha$ does not affect the existence of the cancellation term $c_{2} g_{1}-g_{2} c_{1}$ whose effect is the same as in 4.10 . The analytical derivation for this case would be tedious, however.

\subsubsection{Current Cancellation}

The time waveforms are shown in Fig. 4.16 for the complex $\alpha$ case. It can be seen by comparing the marker values ig1, ig 2 and ie with Fig. 4.12 that both models are identical in terms of nonlinear currents. In fact, all values used for comparison or determination of distortion analysis turn out to be same based on an accuracy of 3 - 4 digits after the decimal point. The statements made in subsection 4.10 .1 
regarding frequency influence, current through the B-E capacitance apply here as well.

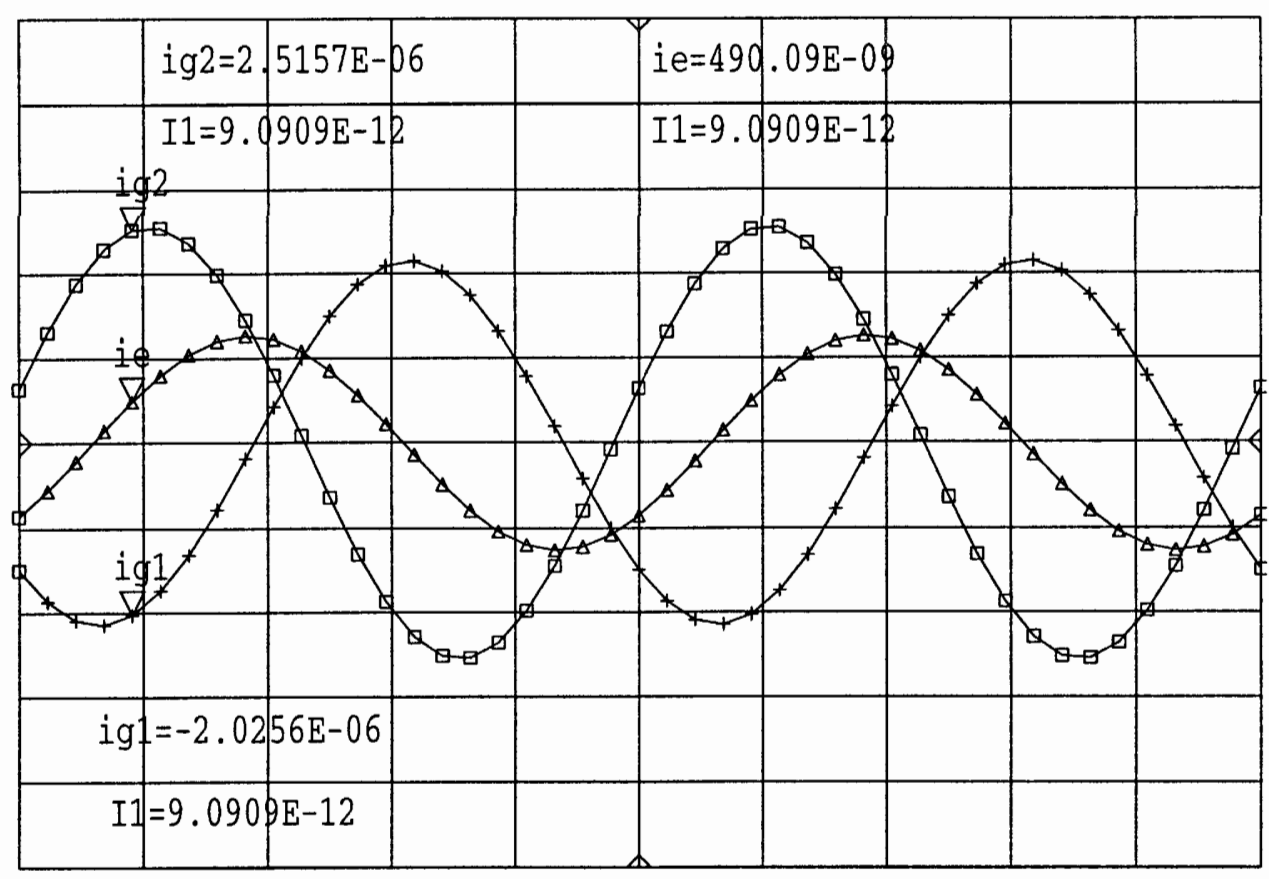

Figure 4.16: $I_{g 1}(2 \omega)+I_{g 2}(2 \omega) \approx I_{e}(2 \omega)$. The marker ig1, ig2 and ie of the time waveform correspond to its counterparts in the frequency domain $I_{g 1}(2 \omega), I_{g 2}(2 \omega)$ and $I_{e}(2 \omega)$, respectively, which is just a different way of stating the same as in the caption of Fig. 4.12. Practically speaking, $I_{g 1}(2 \omega)+I_{g 2}(2 \omega)=I_{e}(2 \omega)$, because the magnitude of the currents steming from the B-E capacitance is small compared to the currents in the onductive portion. But since this model explicitely defines the emitter current through conductance and capacitance one might object that Kirchhoffs current law is not satisfied if the current flowing through the capacitor is neglected and an equal sign is used for the above relationship. Therefore the $\approx$ sign has been used in the caption of Fig.4.16 for the sake of consistency. Case3 


\subsection{Equivalence of Case 2 and Case 3}

By comparing the marker values in Fig. 4.12 and Fiq. 4.16 for case 2 and case 3 the equality of the currents can be seen. It was also mentioned that all other values e.g. nodal voltages used for comparison were identical. This section shows why both models are identical if a change in the definition of emitter current is accompanied by a different modelling of $\alpha . V_{1}(\omega)$ of case 2 is described by:

$$
V_{1, \text { case } 2}(\omega)=V_{1, c 2}(\omega)=\frac{V_{s}}{1+g_{1} R_{b}(1-\alpha)+j \omega c_{1} R_{b}},
$$

where $\alpha=\alpha_{0} . V_{1}(\omega)$ of case 3 is described by:

$$
V_{1, \text { case3 }}(\omega)=V_{1, c 3}(\omega)=\frac{V_{s}}{1+\left(g_{1}+j \omega c_{1}\right) R_{b}(1-\alpha)},
$$

where $\alpha$ is:

$$
\alpha=\frac{\alpha_{0}}{1+j \frac{\omega}{\omega_{c o, \alpha}}}
$$

and $\omega_{c o, \alpha}=\frac{g_{1}}{c_{1}}$. Substituting Eq. 4.78 in Eq. 4.77 and after some manipulation it follows that $V_{1, c 2}(\omega)=V_{1, c 3}(\omega)$, indeed. $V_{2}(2 \omega)$ of case 2 is described by:

$$
V_{2, c 2}(2 \omega)=-\frac{R_{b}\left[I_{g 2}\left(1-\alpha_{0}\right)+I_{c 2}\right]}{1+g_{1} R_{b}\left(1-\alpha_{0}\right)+j 2 \omega c_{1} R_{b}} .
$$

$V_{2}(2 \omega)$ of case 3 is described by:

$$
V_{2, c 3}(2 \omega)=-\frac{R_{b}\left[I_{g 2}(1-\alpha)+I_{c 2}(1-\alpha)\right]}{1+g_{1} R_{b}(1-\alpha)+j 2 \omega c_{1} R_{b}(1-\alpha)},
$$

where $\alpha$ is again given by:

$$
\alpha=\frac{\alpha_{0}}{1+j \frac{2 \omega}{\omega_{c o, \alpha}}} .
$$


Dividing Eq. 4.79 and Eq. 4.80 leads to the expression:

$$
\frac{V_{2, c 2}(2 \omega)}{V_{2, c 3}(2 \omega)}=\frac{g_{1} g_{2}\left(1-\alpha_{0}\right)-j 2 \omega c_{1} g_{2}\left(1-\alpha_{0}\right)-j 2 \omega c_{2} g_{1}+4 \omega^{2} c_{2} c_{1}}{g_{1} g_{2}\left(1-\alpha_{0}\right)-j 2 \omega c_{2} g_{1}\left(1-\alpha_{0}\right)-j 2 \omega c_{1} g_{2}+4 \omega^{2} c_{2} c_{1}} .
$$

Using again $c_{1} g_{2}=c_{2} g_{1}$ this fraction equals unity which sheds new light to the cancellation term $c_{1} g_{2}-c_{2} g_{1}=0$, namely that this term causes the equality of the nodal voltages of case 2 and case 3 .

It turns out that case 2 and 3 are indeed identical. The correspondence between both models is established by the corner frequency relations given in 4.11 . To state this somewhat surprising fact slightly differently:

- if the emitter current is assumed to flow through the conductive part of the base emitter junction only then $\alpha$ is a constant, i.e. independent of frequency.

- if the emitter current is assumed to flow through the conductive and capacitive part of the base emitter junction then $\alpha$ is complex and a function of frequency.

It was also mentioned that the contribution of the nonlinear base emitter capacitance to the magnitude of the emitter current is insignificant. This, in fact, is the justification for the approach taken by Samelis et al. [2]. They focus on cancellation between currents induced by the base collector and base emitter junction, i.e. the total emitter current. The importance of the nonlinear base emitter capacitance stems from the fact that it is instrumental in developing the phase differences of nonlinear voltage drop across the base emitter junction. As shown, these phase differences cause second order current cancellation within the junction resulting in lower total second order emitter current. 


\subsection{Case 4}

The circuit is the same as in case 2 but the emitter resistance $R_{e e}$ is included which entails that the nodal voltages $V_{1}(\omega)$ and $V_{2}(2 \omega)$ are no longer the terminal voltages for the nonlinearity under consideration. Now the phase difference between $\left[V_{t 1}(\omega)\right]^{2}$ and $V_{t 2}(2 \omega)$ defines the cancellation between $I_{g 2}(2 \omega)$ and $I_{g 1}(2 \omega)$.

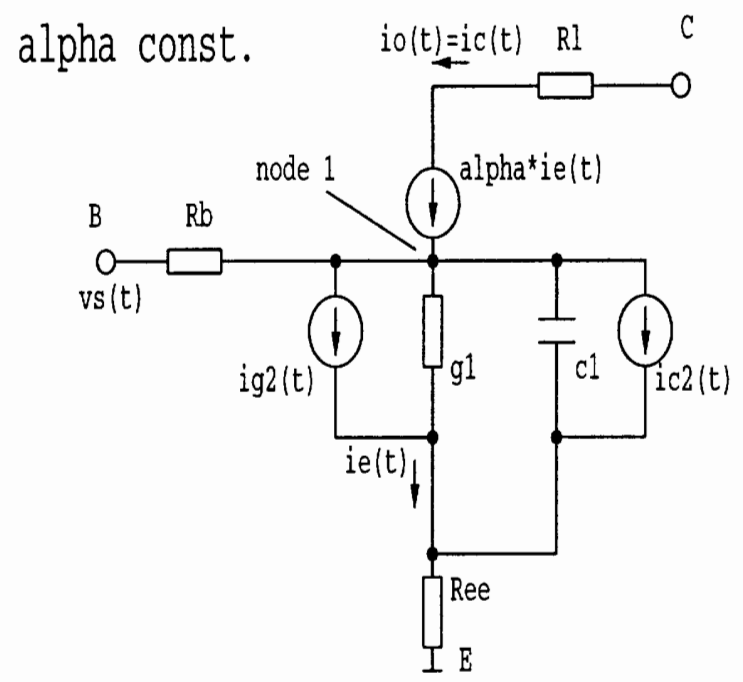

Figure 4.17: Equivalent circuit model for case 4

\subsubsection{Nodal Voltages}

The phase of $V_{1}(\omega)$ is given as:

$$
\phi_{1}=\arctan \frac{\omega c_{1} R_{e e}}{g_{1} R_{e e}+1}-\arctan \frac{\omega c_{1}\left(R_{b}+R_{e e}\right)}{1+g_{1}\left[R_{e e}+R_{b}(1-\alpha)\right]}
$$


and of $V_{2}(2 \omega)$ as:

$$
\begin{aligned}
\phi_{2}= & \pi-\arctan \frac{g_{2}(1-\alpha)}{2 \omega \alpha R_{e e} \underbrace{\left(g_{2} c_{1}-g_{1} c_{2}\right)}_{0}+2 \omega c_{2}} \\
& +\arctan \frac{1+g_{1}\left[R_{e e}+R_{b}(1-\alpha)\right]}{2 \omega c_{1}\left(R_{e e}+R_{b}\right)}-2 \arctan \frac{\omega c_{1}\left(R_{e e}+R_{b}\right)}{1+g_{1}\left[R_{e e}+R_{b}(1-\alpha)\right]} .
\end{aligned}
$$

It follows then for $\phi_{\text {diff }}$ :

$$
\begin{aligned}
\phi_{\text {diff }}= & \pi-\arctan \frac{g_{2}(1-\alpha)}{2 \omega c_{2}}-2 \arctan \frac{\omega c_{1} R_{e e}}{g_{1} R_{e e}+1} \\
& +\arctan \frac{1+g_{1}\left[R_{e e}+R_{b}(1-\alpha)\right]}{2 \omega c_{1}\left(R_{e e}+R_{b}\right)} .
\end{aligned}
$$

The negative feedback of $R_{e e}$ reverses the frequency impact on the phase shift for the nodal voltages with respect ${ }^{19}$ to case 2 . So far $\omega \rightarrow \infty$ caused $\phi_{\text {diff }} \rightarrow \pi$. Now $\omega \rightarrow \infty$ causes $\phi_{\text {diff }}, \phi_{1}, \phi_{2} \rightarrow 0$. For example, at $f=10 \cdot 10^{15} \mathrm{~Hz}$ MAPLE and MDS give $\phi_{1}=-0.000457^{\circ}, \phi_{2}=0.000275^{\circ}$ and $\phi_{\text {diff }}=0.0012^{\circ}$. These trends are illustrated in Fig.4.18.

\subsubsection{Terminal Voltages}

The terminal voltages for the nonlinearity are the nodal voltages minus the voltage drop across $R_{e e}$. In analogy to the nodal voltages, three phases $\gamma_{1}, \gamma_{2}$ and $\gamma_{\text {diff }}=\gamma_{2}-2 \gamma_{1}$ for $V_{t 1}(\omega), V_{t 2}(2 \omega)$ and $V_{t 2}(2 \omega)-\left[V_{t 1}(\omega)\right]^{2}$ are defined. The phase of $V_{t 1}(\omega)$ is given as:

$$
\gamma_{1}=-\arctan \frac{\omega c_{1}\left(R_{b}+R_{e e}\right)}{1+g_{1}\left[R_{e e}+R_{b}(1-\alpha)\right]}
$$

\footnotetext{
${ }^{19}$ When comparing Fig. 4.11 and Fig. 4.18 , note that a value of $2 \pi$ was added to all graphs in Fig. 4.18 because MAPLE was unable to produce a log plot for $\phi_{1}$. In fact, it complained that it cannot evaluate a boolean. This is probably due to the proximity of $\phi_{1}$ to zero. This problem was circumvented in other cases where $\phi_{1}$ is also close to zero. Unfortunately, the little trick did not work in this particular case.
} 


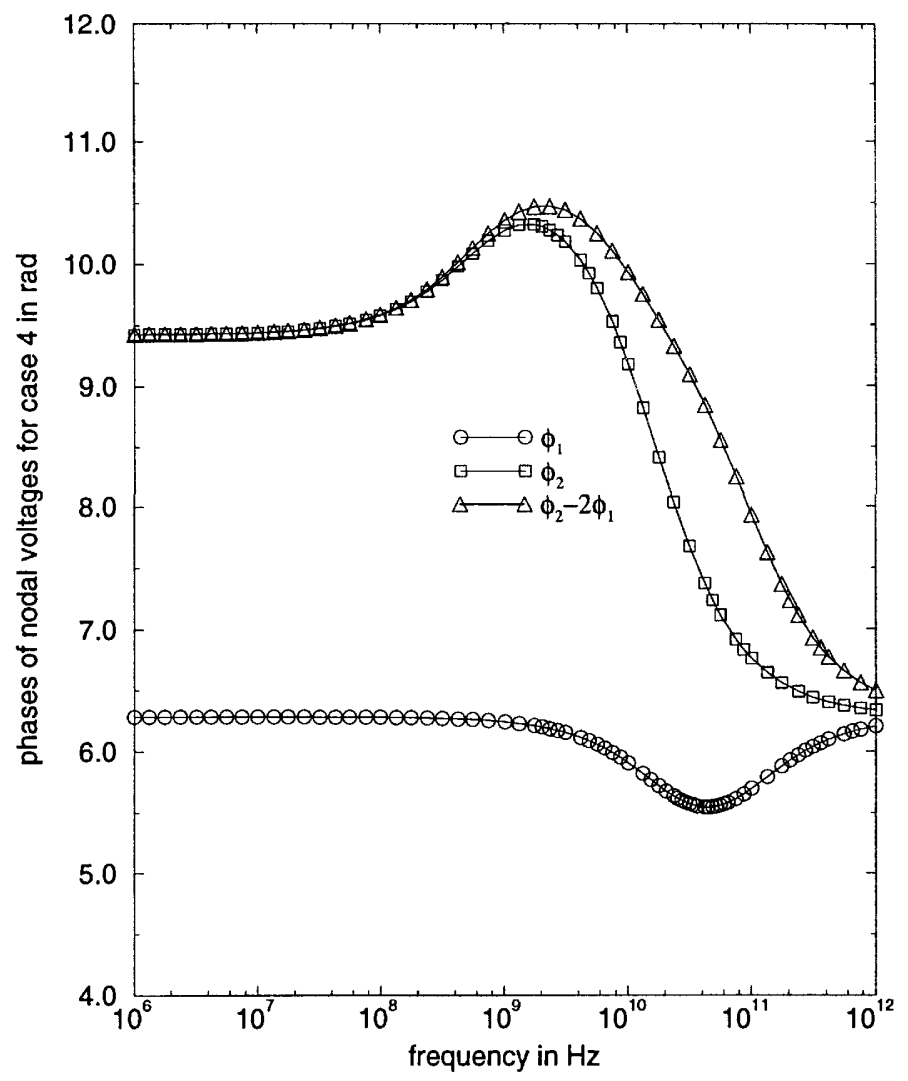

Figure 4.18: The negative feedback of $R_{e e}$ reverses the frequency impact on the phase shift for the nodal voltages with respect to case 2, Fig. 4.11.

and of $V_{t 2}(2 \omega)$ as:

$$
\begin{aligned}
\gamma_{2}= & \arctan \frac{A+B}{C+D}-2 \arctan \frac{\omega c_{1}\left(R_{b}+R_{e e}\right)}{1+g_{1}\left[R_{e e}+R_{b}(1-\alpha)\right]} \\
& -\arctan \frac{2 \omega c_{1}\left(R_{b}+R_{e e}\right)}{1+g_{1}\left[R_{e e}+R_{b}(1-\alpha)\right]} \\
& +\arctan \frac{1+g_{1}\left[R_{e e}+R_{b}(1-\alpha)\right]}{2 \omega c_{1}\left(R_{b}+R_{e e}\right)}+\pi,
\end{aligned}
$$

where

- $A=g_{2}\left[R_{e e}+R_{b}(1-\alpha)\right]-4 \omega^{2} c_{2} c_{1}\left(R_{e e}+R_{b}\right)^{2}$

- $B=g_{2} g_{1}\left[2 R_{b} R_{e e}(1-\alpha)+R_{e e}^{2}+R_{b}^{2}-\alpha R_{b}^{2}(2-\alpha)\right]$ 
- $C=-2 \omega c_{2}\left(R_{e e}+R_{b}\right)$

- $D=\left(g_{1} c_{2}+g_{2} c_{1}\right)\left[\alpha R_{b} R_{e e}-R_{b}^{2}(1-\alpha)-R_{e e}^{2}-2 R_{b} R_{e e}\right]$.

The phase difference is given by:

$$
\begin{aligned}
\gamma_{d i f f}= & \arctan \frac{A+B}{C+D}-\arctan \frac{2 \omega c_{1}\left(R_{b}+R_{e e}\right)}{1+g_{1}\left[R_{e e}+R_{b}(1-\alpha)\right]} \\
& +\arctan \frac{1+g_{1}\left[R_{e e}+R_{b}(1-\alpha)\right]}{2 \omega c_{1}\left(R_{b}+R_{e e}\right)}+\pi .
\end{aligned}
$$

By observing eqs. 4.86 and 4.87 it can be seen that $\gamma_{2}$ holds precisely twice the value of $\gamma_{1}$ and the argument of this arctan function recurrs in two other arctan functions at the second harmonic once inverted and once not. The above equations are presented in Fig.4.19. The emitter resistance eliminates the overshoot of $\angle V_{t 2}(2 \omega)$ immediately before the fall-off. As shown, this brings the phase difference closer to $\pi$ in this range with respect to case 2 . This observation suggests that $R_{e e}$ improves cancellation. This issue will be revisited in section 4.15 .

The emitter resistance affects the cancellation term $c_{1} g_{2}-c_{2} g_{1}$. If the terms are arranged differently ${ }^{20}$ while deriving the analytical expressions for $\gamma_{2}$, terms like

$$
\begin{aligned}
& \text { - } 2 \omega R_{b}^{2}\left(\alpha_{0} c_{2} g_{1}-g_{2} c_{1}\right) \\
& \text { - } 2 \omega R_{b}^{2}\left(\alpha_{0} c_{1} g_{2}-g_{1} c_{2}\right)
\end{aligned}
$$

show up indicating a slightly weaker cancellation mechanism of this kind. The importance of these terms is very similiar to the one outlined in 4.10 . Here they hide

\footnotetext{
${ }^{20}$ This makes the analytical derivation more involved, however. There are, of course, other terms involving the cancellation term but these are the ones which come closest to the original expression.
} 


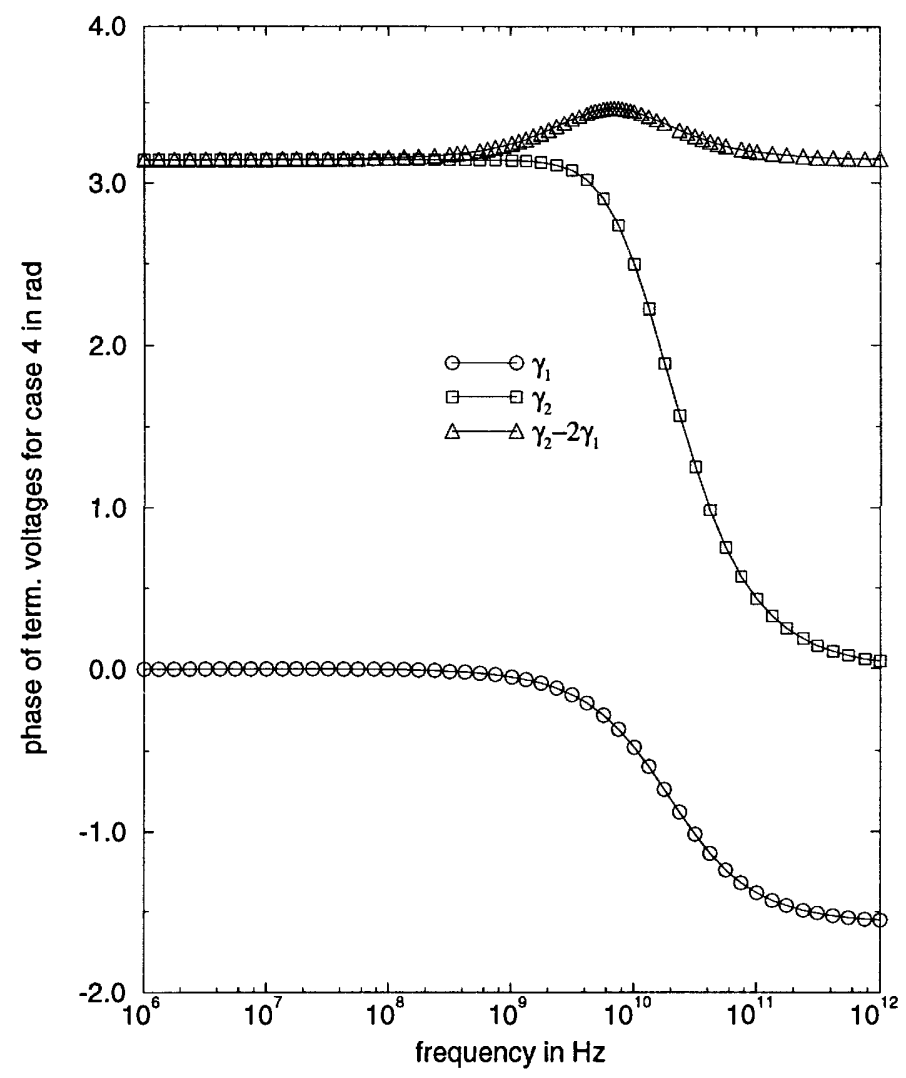

Figure 4.19: The crucial difference to case 2 is that $R_{e e}$ eliminates the overshoot of $\gamma_{2}$ immediately before the fall-off. Accordingly, $\gamma_{d i f f}$ is closer to $\pi$ in this range.

in the factor D in Eq. 4.88. If $f \rightarrow \infty$ the first term in Eq. 4.88 approaches asymptotically $-\frac{\pi}{2}$, the second $\frac{\pi}{2}$ and the third zero, thus $\gamma_{d i f f} \rightarrow \pi$. The cancellation terms (although weaker than in 4.10 and 4.11 ) speed up the approach towards $-\frac{\pi}{2}$ balancing out the second term whose denominator is fairly small, resulting in a fast rise towards $\frac{\pi}{2}$. 


\subsubsection{Current Cancellation}

The time waveforms are shown in Fig.4.20. It can be seen by comparing the marker values with Fig.4.12 that the emitter resistance reduces the magnitude of the nonlinear currents dramatically which improves IP2 according to the reasoning outlined in section 4.10.1.

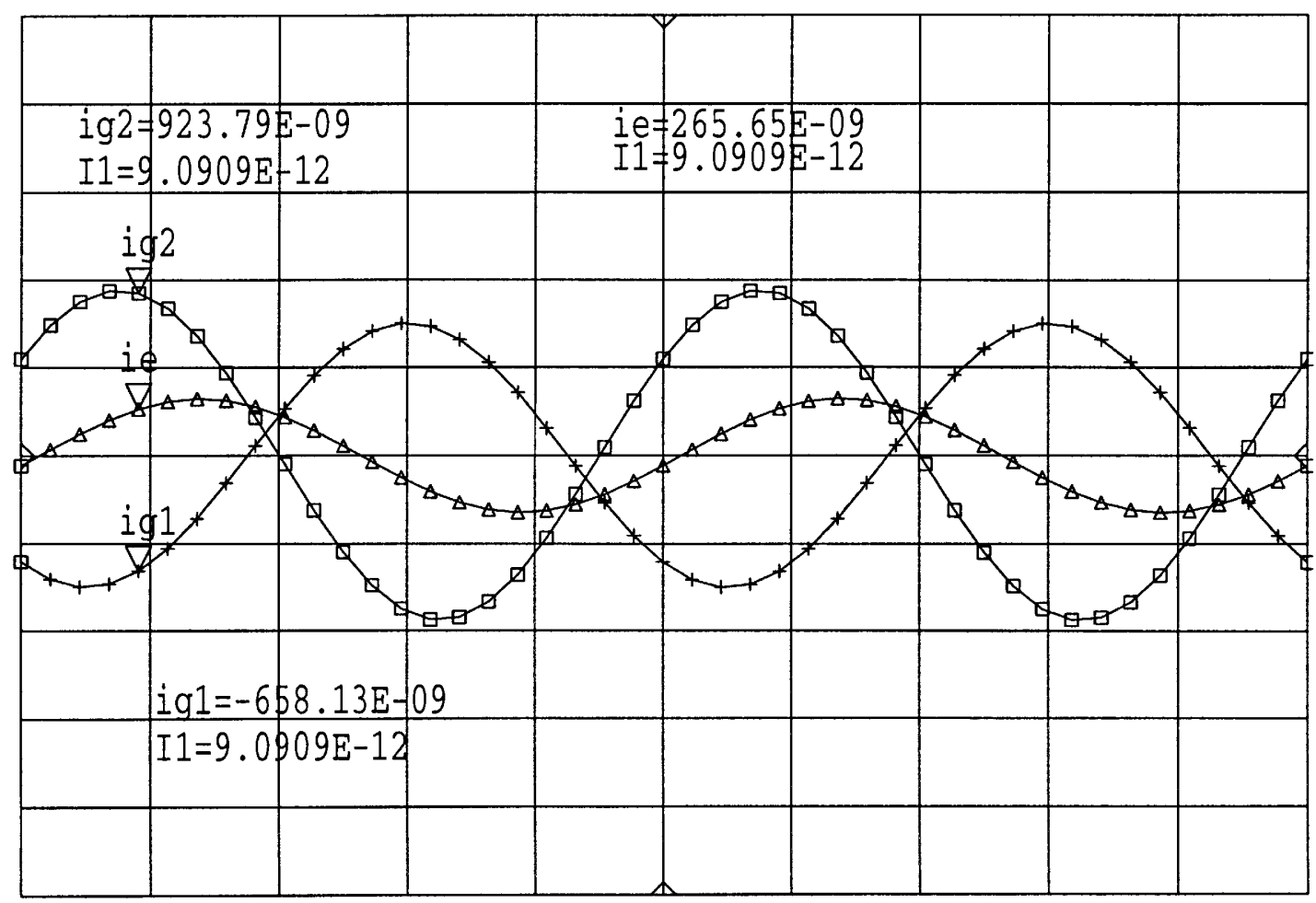

Figure 4.20: Observe the greatly reduced magnitude of the nonlinear currents flowing through the B-E conductance due to $R_{e e}$ in case 4 with respect to case 2 and 3 $\left(I_{g 1}(2 \omega)+I_{g 2}(2 \omega)=I_{e}(2 \omega), \mathrm{f}=10 \mathrm{GHz}\right.$, case 4$)$.

In summary, $R_{e e}$ influences the IP2 in two ways: It reduces the magnitude of the 
nonlinear currents in B-E conductance and it eliminates the overshoot of $\angle V_{t 2}(2 \omega)$ immediately before the fall-off which improves cancellation.

\subsection{Case 5}

Since it has already been shown that the two ways of modeling the base emitter junction are equivalent, and since adding a series resistor should not affect this, case 5 is treated briefly. Just the equations for the phases of the nodal voltages for the low frequency and the general case are given. Because the general case 5 may be regarded as the most difficult, the MAPLE worksheet for this case ${ }^{21}$ is given in appendix F.

The equivalence of both models when the emitter resistance is included has, however, been checked numerically and also by MAPLE. The expected agreement was found.

Case 5 uses the same definition for the emitter current as in 4.11 but as in 4.13 the emitter resistance $R_{e e}$ included. The circuit is shown in Fig.4.21. The voltages $V_{1}(\omega)$ and $V_{2}(2 \omega)$ are the nodal voltages at node 1 .

\subsubsection{Low frequency}

The phase of $V_{1}(\omega)$ is then given as:

$$
\phi_{1}=\arctan \frac{1+g_{1}\left[R_{e e}+R_{b}(1-\alpha)\right]}{\omega c_{1}\left[R_{b}(1-\alpha)+R_{e e}\right]}-\arctan \frac{g_{1} R_{e e}+1}{\omega c_{1} R_{e e}}
$$

\footnotetext{
${ }^{21}$ The calculation is at $f=1 \mathrm{GHz}$. Only the last part has been executed to show the results. The remaining part has been left in general form to illustrate the steps of the nonlinear current method.
} 


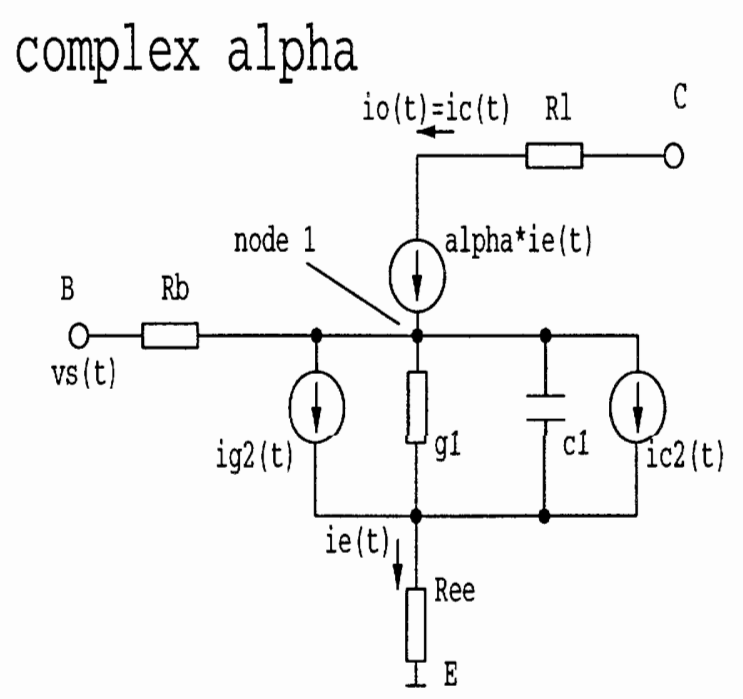

Figure 4.21: Equivalent circuit model for case 5. Note that the $I_{e}$ is defined through B-E conductance and capacitance.

and of $V_{2}(2 \omega)$ as:

$$
\begin{aligned}
\phi_{2}= & -\arctan \frac{g_{2}}{2 \omega c_{2}}+\arctan \frac{1+g_{1}\left[R_{e e}+R_{b}(1-\alpha)\right]}{2 \omega c_{1}\left[R_{e e}+R_{b}(1-\alpha)\right]} \\
& +2 \arctan \frac{1+g_{1}\left[R_{e e}+R_{b}(1-\alpha)\right]}{\omega c_{1}\left[R_{e e}+R_{b}(1-\alpha)\right]} .
\end{aligned}
$$

It follows then for $\phi_{\text {diff }}$ :

$$
\begin{aligned}
\phi_{\text {diff }}= & -\arctan \frac{g_{2}}{2 \omega c_{2}}+\arctan \frac{1+g_{1}\left[R_{e e}+R_{b}(1-\alpha)\right]}{2 \omega c_{1}\left[R_{e e}+R_{b}(1-\alpha)\right]} \\
& +2 \arctan \frac{g_{1} R_{e e}+1}{\omega c_{1} R_{e e}} .
\end{aligned}
$$

\subsubsection{Complex $\alpha$}

The phase of $V_{1}(\omega)$ is given as:

$$
\phi_{1}=\arctan \frac{\omega c_{1} R_{e e}}{g_{1} R_{e e}+1}+\arctan \frac{\omega}{\omega_{c o, \alpha}}
$$


$-\arctan \frac{\omega\left\{1+g_{1}\left[R_{e e}+R_{b}\right]+\omega_{c o, \alpha} c_{1}\left[R_{e e}+R_{b}\left(1-\alpha_{0}\right)\right]\right\}}{\omega_{c o, \alpha}+\omega_{c o, \alpha} g_{1}\left[R_{e e}+R_{b}\left(1-\alpha_{0}\right)\right]-\omega^{2} c_{1}\left(R_{e e}+R_{b}\right)}$.

The phase of $V_{2}(2 \omega)$ is given as:

$$
\begin{aligned}
\phi_{2}= & 2 \arctan \frac{\omega c_{1} R_{e e}}{g_{1} R_{e e}+1}+2 \arctan \frac{\omega}{\omega_{c o, \alpha}}-\frac{1}{2} \pi \\
& -\arctan \frac{2 \omega\left[c_{2} \omega_{c o, \alpha}\left(1-\alpha_{0}\right)+g_{2}\right.}{-\omega_{c o, \alpha} g_{2}\left(1-\alpha_{0}\right)+4 \omega^{2} c_{2}}+2 \arctan \frac{g_{1} R_{e e}+1}{\omega c_{1} R_{e e}} \\
& -\arctan \frac{-\omega_{c o, \alpha}\left\{1+g_{1}\left[R_{e e}+R_{b}\left(1-\alpha_{0}\right)\right]\right\}+4 \omega^{2} c_{1}\left(R_{b}+R_{e e}\right)}{2 \omega\left\{1+g_{1}\left(R_{b}+R_{e e}\right)+\omega_{c o, \alpha} c_{1}\left[R_{e e}+R_{b}\left(1-\alpha_{0}\right)\right]\right\}} .
\end{aligned}
$$




\subsection{Influence of various Parameters}

\subsubsection{Impact of $R_{e e}$}

Fig.4.22 shows that the phase difference and therefore the cancellation improves if $R_{e e}$ is included which supports Wang's et al. [5] findings. Fig. 4.23 shows that

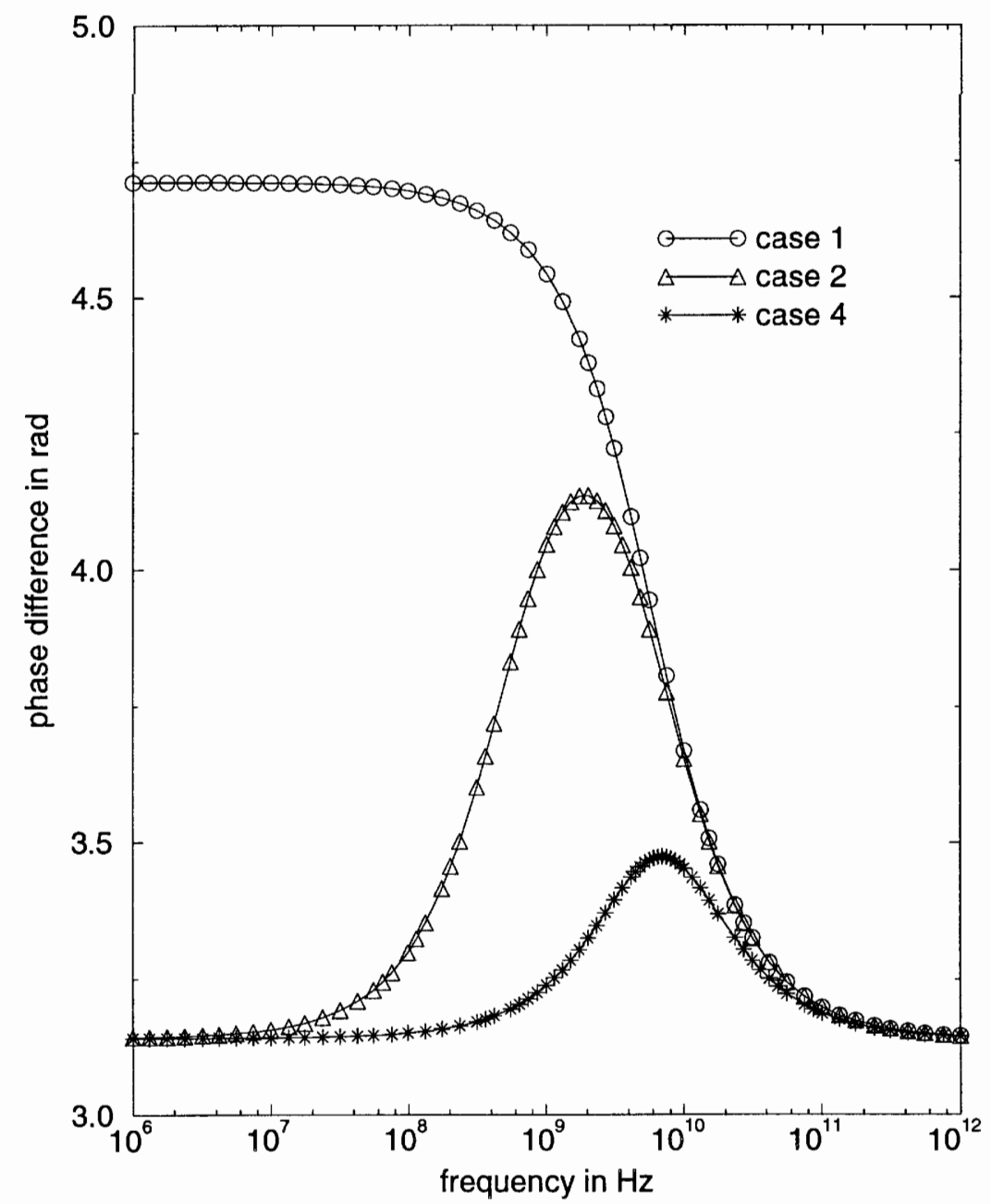

Figure 4.22: Phase difference summarized for case 1, 2 and 4

the phase difference and therefore the cancellation improves if $R_{e e}$ increases. This actually conflicts with [5]. They found that although emitter and base resistances 
linearize the HBT and hence reduce ${ }^{22}$ IMD2 and IMD3 by providing negative feedback, $R_{e e}$ has no affect on the cancellation. On the other hand, Fig. 4.23 suggests clearly a frequency range, where the cancellation is degraded. To test this finding a

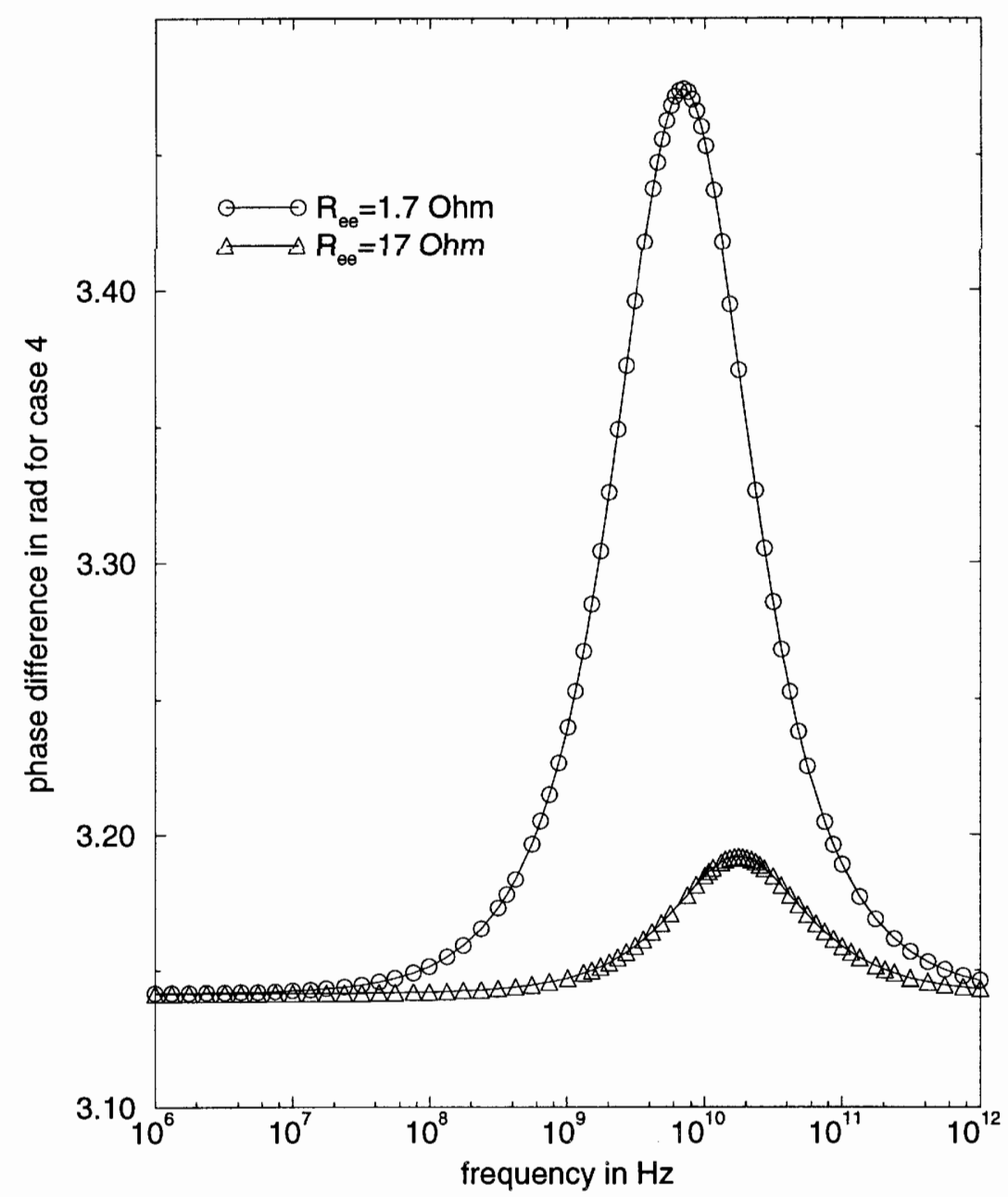

Figure 4.23: Impact of $R_{e e}$ on phase difference

simulation was run at $f=10 \mathrm{GHz}$, which falls in the range of degraded cancellation for the equivalent circuit with $R_{e e}=1.7 \Omega$, but the emitter resistance was increased by a factor of ten. Almost perfect cancellation for the case $R_{e e}=17 \Omega$ is shown in

\footnotetext{
${ }^{22}$ In particular, their IMD3 reduces by $10 \mathrm{~dB}$ if emitter and base resistances are included.
} 
Fig. 4.24 which indicates that $R_{e e}$ not only influences the IP2 by decreasing the magnitude of the second order currents but also by bringing their phase difference much closer to $\pi$. The IP2s associated with $R_{e e}=1.7 \Omega$ and $R_{e e}=17 \Omega$ are 29 and $41 \mathrm{dBm}$, respectively. 


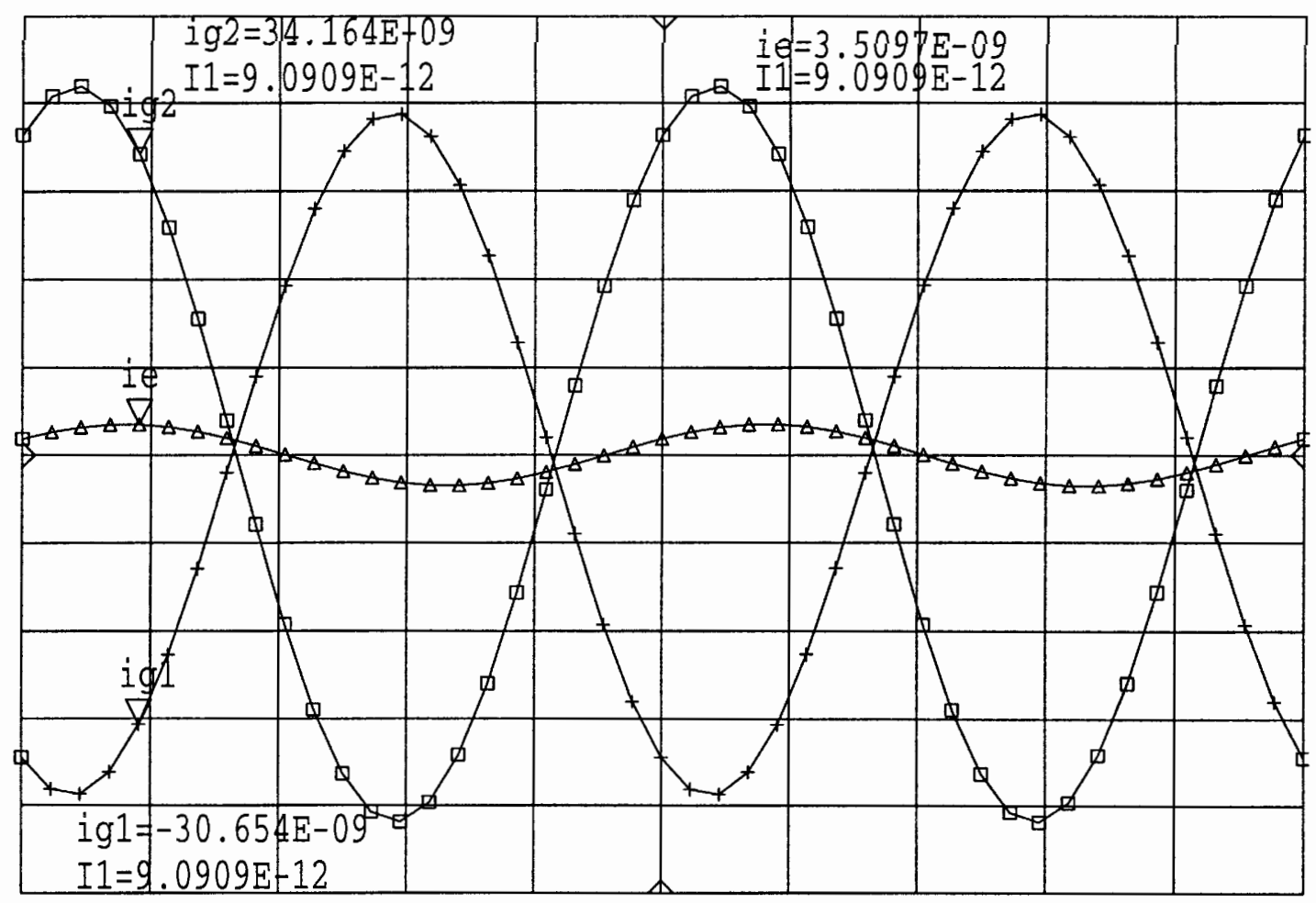

Figure 4.24: Impact of $R_{e e}$ on cancellation. The marker ig1, ig2 and ie denote the time waveforms of the phasors $I_{g 1}(2 \omega), I_{g 2}(2 \omega)$ and $I_{e}(2 \omega)$, respectively and have been set at $\mathrm{t}=9.09$ ps. $I_{g 1}(2 \omega)$ and $I_{g 2}(2 \omega)$ are the currents through the linear $\left(g_{1}\right)$ and the nonlinear part $\left(g_{2}\right)$ of the nonlinear conductance g. $I_{e}(2 \omega)$ is the emitter current. All currents are at the second harmonic. Case 4, larger $R_{e e}$ 


\subsubsection{Impact of $\alpha$ on Phase Difference}

Fig. 4.25 shows that the phase difference and therefore the cancellation improves if $\alpha$ decreases. This result is somewhat surprising. Caution when drawing conclu-

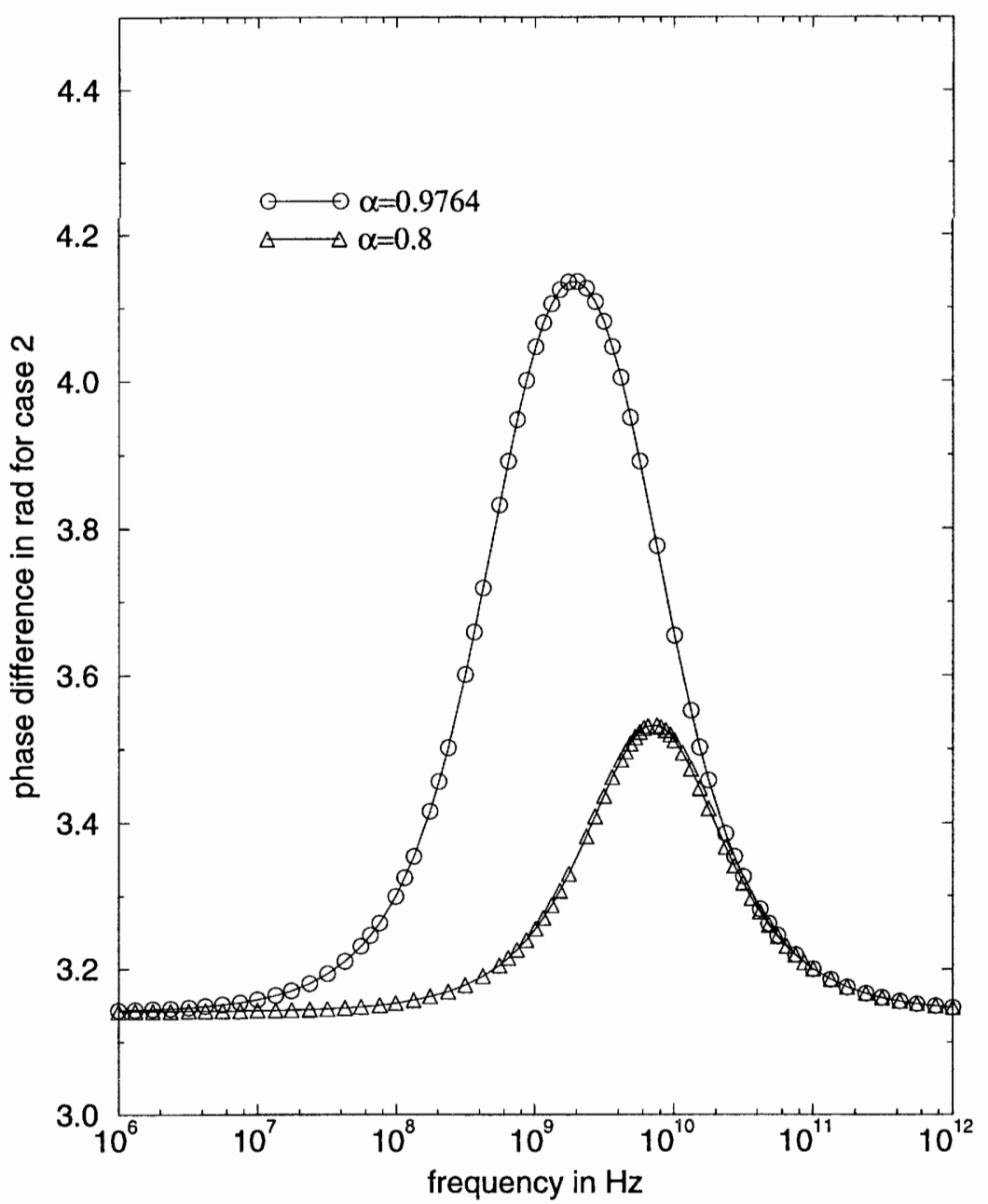

Figure 4.25: Impact of $\alpha$

sions is recommended here because the time delay $t_{d}$ has not been considered. The analytical derivations in this thesis modeled $\alpha$ either as a constant $\alpha=\alpha_{0}$ or as explicitely frequency dependent $\alpha=\frac{\alpha_{0}}{1+j \frac{\omega}{\omega_{\mathrm{co}, \alpha} \alpha}}$. Also, $\alpha$ is not broken down to its 
components, such as:

$$
I_{c}=\alpha_{1} I_{e}\left(t-t_{d}\right)+\alpha_{2} I_{e}^{2}\left(t-t_{d}\right)+\alpha_{3} I_{e}^{3}\left(t-t_{d}\right)
$$

as suggested in [6]. The following section 4.16 will reveal that the time delay has a complicated and crucial impact on the IP2 graph. It is mainly responsible for the saturation of IP2 at higher frequencies. It is also remarkable that IP2 and IP3 changed by $6-10 \mathrm{dBm}$ if $t_{d}$ was altered by only a few pico seconds. Clearly, this subject deserves further attention. 


\subsection{IP2}

The second order intercept point which was defined in Fig. 2.1 is the final measurement for a device's linearity. It is now summarized how the cancellation mechanism and parameter sensitivities influence the IP2. Since this thesis focused more on one tone excitation, the IP2 steming from the second harmonic distortion product is considered. It may be mentioned that the IP2 for a single tone excitation equals approximately the IP2 calculated for the second order intermodulation product if the excitation is separated by a small delta frequency. Fig. 4.26 displays the IP2s of different models analyzed in this thesis. Here is a guide for the legends in Fig. 4.26:

1. "case 3 " and "case 5" are the models investigated in this thesis. The original values $\alpha_{0}=0.9764$ and $R_{e e}=1.7 \Omega$ were used for these simulations.

2. "gen. model no $t_{d}$ " stands for general model without considering the time delay. This model was the starting point and the corresponding circuit is shown in Fig. 4.1.

3. "gen. w. model $t_{d}$ " is the same as 2 but the time delay is included.

4. "fit. coeff. no $t_{d}$ " is again the general model from Fig. 4.1 but the Taylor series coefficients are replaced by fitted coefficients. It is obvious from the plot why the coefficients had to be adjusted to match the simulation results to measurements.

5. "fit. coeff. w. $t_{d}$ " is the same as 4 but the time delay is considered. 


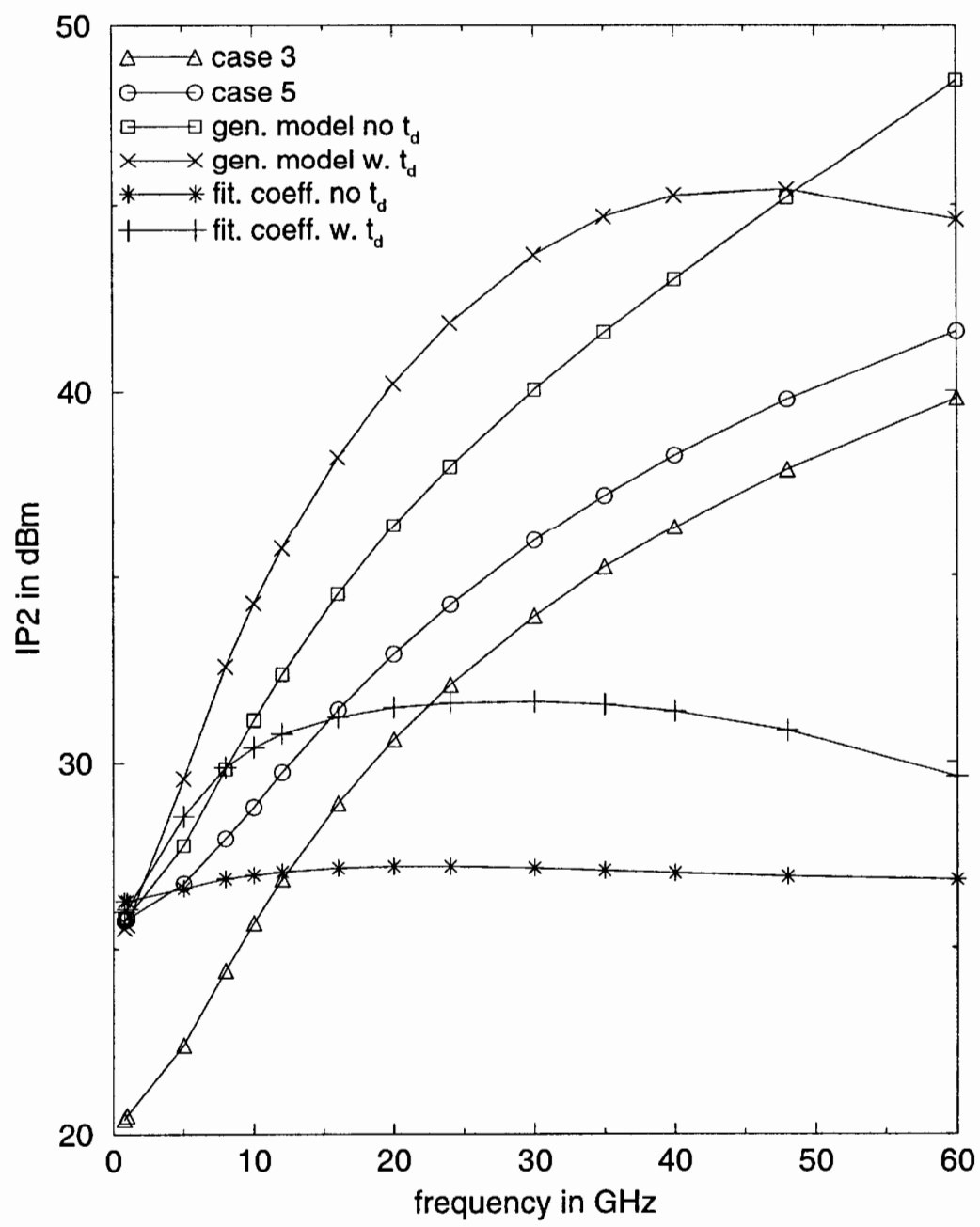

Figure 4.26: IP2 for various models.

Fig. 4.26 displays that the IP2 for case 5 is higher than the one for case 3 . This was expected due to $R_{e e}$ which reduces the magnitude of the nonlinear currents and it improves cancellation. It was also found that higher frequency improves cancellation and hence IP2. This is true for the investigated cases, i.e. case 3 and 5 are shown but the same result was obtained for case 2 and 4 . If the time delay is not included the general model obeys this dependence as well. It is the explicit consideration of $t_{d}$ which leads to the saturation of IP2 at high frequencies. 
Fig. 4.26 also indicates that the cancellation mechanism is weaker in real devices because the IP2 using the fitted coefficients is lower. The Taylor coefficient $c_{2}$ is larger by a factor of two with respect to the fitted coefficient $c_{2}$. This overemphasizes the strength of the nonlinear capacitance with respect to the situation in real devices and hence cancellation. As already mentioned in [6] the Taylor series was an expansion of the nonlinear charge voltage relation which was assumed to be diffusion. This is not necessarily true because it neglects the depletion capacitance which, if included, reduces the strength of the capacitive nonlinearity. To reflect this fact, the coefficient $c_{2}$ had to be reduced which turns the Taylor coefficient into a fitted one. This calls for a more sophisticated modelling of the base emitter junction. Especially since the influence of the depletion part will increase if the HBT is driven in class $\mathrm{B}$ or other more nonlinear modes of operation.

\subsection{Review of Approximations}

In this section, the several approximations made to simplify the general circuit in Fig. 4.1 are assessed. The terminal voltages across the nonlinear B-E junction will be compared for this purpose which is shown in Tab. 4.3. Recall that the terminal voltages in case 1 and 2 are identical with the nodal voltages. Comparing case 1 and 2 justifies the approximation $\alpha \approx 1$ which links both cases. Neglecting $R_{e e}$ which connects case 2 and 4 is rather crude in terms of magnitude. It may be tolerated for phase investigations because, since $\angle V_{t 1}(\omega)$ and $\angle V_{t 2}(2 \omega)$ change at the same rate, it is the phase difference responsible for cancellation. Excluding the parasitics which 


\begin{tabular}{|l|l|r|}
\hline \hline & $V_{t 1}(\omega)$ in $\mu V$ & $V_{t 2}(2 \omega)$ in $\mu V$ \\
\hline case 1 & $762.3 \angle-40.3^{\circ}$ & $4.47 \angle 129.8^{\circ}$ \\
\hline case 2 & $721.2 \angle-37.8^{\circ}$ & $3.9 \angle 134.1^{\circ}$ \\
\hline case 4 & $437.8 \angle-27.2^{\circ}$ & $1.37 \angle 143.5^{\circ}$ \\
\hline gen. model w/o $t_{d}$ & $395.7 \angle-33.5^{\circ}$ & $1.71 \angle 127.7^{\circ}$ \\
\hline gen. model with $t_{d}$ & $310.5 \angle-45^{\circ}$ & $0.78 \angle 100.5^{\circ}$ \\
\hline
\end{tabular}

Table 4.3: Evaluating the different approximations by comparing the terminal voltage across the $\mathrm{B}-\mathrm{E}$ junction

is the approximation relating case 4 and the general model may be acceptable. It should be pointed out, nevertheless, that the trend of decreasing $\angle V_{t 1}(\omega)$ is reversed by dropping the linear assumed parts such as base collector capacitance. The time delay $t_{d}$ plays a very subtle role. Time delay is traditionally associated with phase shift or phase delay but it also affects the magnitude. The approximations here have been addressed with respect to the terminal voltages. Subsequent work may choose other criteria, e.g. the excitation voltage may be adjusted such that the terminal voltage is the same for each case. The currents through the B-E junction could serve as another frame of reference.

In summary, Tab. 4.3 indicates that the approximations relating case 1,2 , and 4, which are the major focus of this thesis, may be tolerated. The approximations explain the need to adjust parameters to match distortion measurements. 


\subsection{Checking the Basic Equation of the Nonlin-}

\section{ear Current Method}

A set of equation is suggested in section 4.9 which is applicable if the calculation is limited to third order mixing products and the power series representing the nonlinearity can be truncated after the cubic term. Clearly, eqs. 4.8 and 4.9 are approximations which are checked as follows: The $\mathrm{RC}$ input circuit, which was given in Fig.4.3 is simulated with MDS using different amplitudes for the input signal and the voltage components $v_{1}, v_{2}$ and $v_{3}$ are obtained. The full expression, as given in section 4.4 and appendix B, for eqs. 4.8 and 4.9 has been evaluated using MAPLE and compared with Maas approximation. The comparison is shown in Table 4.4. $V_{\text {in }}$ is the amplitude of the input signal. $v_{1}, v_{1}, v_{3}$ are the voltage components across the nonlinearity, in this case the capacitance. Remember, that $V_{i n}=v_{R b}+v_{1}+v_{2}+v_{3}$. The results indicate that at an input amplitude of $10 \mathrm{mV}$ small disagreement begins to occur.

\subsubsection{Disagreement between MDS and MAPLE}

A similiar disagreement is also found by a direct comparison between MDS and MAPLE. For example, simulating case 2 again at $\mathrm{f}=10 \mathrm{GHz}$ and executing the appropriate MAPLE worksheet illustrates this disagreement. The results are summarized in Table 4.5. It shows the input voltage with its associated power level. The angles are given in radians. The MAPLE computed phase does not change because 


\begin{tabular}{|c|c|c|c|}
\hline & & full MAPLE & approximation \\
\hline \multirow{2}{*}{$\begin{array}{l}V_{i n}=1 \mathrm{~V}, v_{1}=0.374 \mathrm{~V} \\
v_{2}=0.073 \mathrm{~V}, v_{3}=0.024 \mathrm{~V}\end{array}$} & $v^{2}$ & 0.221841 & 0.19448 \\
\hline & $v^{3}$ & 0.104487 & 0.05231 \\
\hline \multirow{2}{*}{$\begin{array}{l}V_{i n}=100 m V, v_{1}=74 m V \\
v_{2}=14 m V, v_{3}=3.817 m V\end{array}$} & $v^{2}$ & 0.00843 & 0.007548 \\
\hline & $v^{3}$ & $774.05 \cdot 10^{-6}$ & $405.0 \cdot 10^{-6}$ \\
\hline \multirow{2}{*}{$\begin{array}{l}V_{\text {in }}=10 m V, v_{1}=7.579 m V \\
v_{2}=160.9 \mu V, v_{3}=5.579 n V\end{array}$} & $v^{2}$ & $599.06 \cdot 10^{-6}$ & $598.8 \cdot 10^{-6}$ \\
\hline & $v^{3}$ & $463.6678 \cdot 10^{-9}$ & $435.347 \cdot 10^{-9}$ \\
\hline \multirow{2}{*}{$\begin{array}{l}V_{\text {in }}=1 \mathrm{mV}, v_{1}=758.1 \mu \mathrm{V} \\
v_{2}=1.611 \mu V, v_{3}=5.6 n V\end{array}$} & $v^{2}$ & $577.169 \cdot 10^{-9}$ & $577.158 \cdot 10^{-6}$ \\
\hline & $v^{3}$ & $438.485 \cdot 10^{-12}$ & $435.692 \cdot 10^{-12}$ \\
\hline
\end{tabular}

Table 4.4: Checking approximation

the coefficients' $\left(g_{1,2}\right.$ and $c_{1,2}$ ) dependence on $I_{e}$ is not considered in MAPLE. This problem has been adddressed in section 4.9.2 where the influence of the amplitude on the phase was illustrated. 


\begin{tabular}{|l|l|l|r|}
\hline excitation & nodal voltage & MDS & MAPLE \\
\hline \multirow{2}{*}{$\begin{array}{l}V_{\text {in }}=100 \mathrm{mV} \\
P_{\text {in }} \approx 40 \mathrm{dBm}\end{array}$} & $V_{1}$ & $0.061, \angle-0.77$ & $0.072, \angle-0.66$ \\
\cline { 2 - 4 } & $V_{2}$ & $0.013, \angle 1.89$ & $0.039, \angle 2.34$ \\
\hline $\begin{array}{l}V_{\text {in }}=10 \mathrm{mV} \\
P_{\text {in }} \approx 21 \mathrm{dBm}\end{array}$ & $V_{1}$ & $7.19 \cdot 10^{-3}, \angle-0.658$ & $7.21 \cdot 10^{-3}, \angle-0.659$ \\
\cline { 2 - 4 } & $V_{2}$ & $385.6 \cdot 10^{-6}, \angle 2.34$ & $390.7 \cdot 10^{-6}, \angle 2.34$ \\
\hline \hline
\end{tabular}

Table 4.5: Disagreement between MDS and MAPLE 


\section{Chapter 5}

\section{Full IP3 Simulation in MDS}

\subsection{The Self Defined Device in MDS Harmonic}

\section{Balance Simulations}

Maas and Nelson [6] suggest a straightforward model which shows that complete nonlinear current cancellation may theoretically occur because the resistive and capacitive induced currents in the base emitter junction are 180 degrees out of phase. They, therefore, can afford to omit the diode modelling of the base collector junction and a current generator modeling reverse injection into the emitter. In [6] the relationship between collector and emitter current is given as:

$$
I_{c}=\alpha_{1} I_{e}\left(t-t_{d}\right)+\alpha_{2} I_{e}^{2}\left(t-t_{d}\right)+\alpha_{3} I_{e}^{3}\left(t-t_{d}\right)
$$

This represents a nonlinear system (i.e. it has a square and a cubic term) with a constant time delay $t_{d}$. Teeter et al. [3] suggest (p.843) that this time delay is mainly caused by the collector transit time and dominates the base current at millimeter wave frequencies. MDS allows for simulating such a system via a "self-defined" 
device (SDD).

In a two tone HB simulation the MNS calculates the two fundamentals or tones $f_{1}$ and $f_{2}$ according to:

$$
f_{1,2}=f_{\text {center }} \pm \frac{\Delta f}{2}
$$

During a HB simulation the MNS supplies the SDD with the spectrum $I_{e}(\omega)$ of the port current ${ }^{1}$ and asks the SDD for the spectrum of $I_{c}(\omega)$

$$
I_{e}(\omega) \stackrel{F F T-1}{\Longrightarrow} i_{e}(t) \stackrel{i_{c}=f\left(i_{e}\right)}{\Longrightarrow} i_{c}(t) \stackrel{F F T}{\Longrightarrow} \tilde{I}_{c}(\omega) \stackrel{\text { weighting }}{\Longrightarrow} I_{c}(\omega)
$$

The input spectrum is being converted into a sample time waveform via inverse Fast Fourier Transformation. An important parameter to consider is called oversample. The default value is 1 . Increasing the parameter oversample improves accuracy and convergence in general, but it also increases computation time and data storage. The nonlinearity is evaluated in the time domain point by point along the time waveform. The weighting function is being applied in the frequency domain. Fig. 5.1 shows how eq. 5.1 is implemented in MDS. Note that eq. 5.1 has been separated into 3 equations for port 2. This is necessary because each term in eq. 5.1 will be multiplied by a different weight factor. The parameters a1, a2, and a3 in Fig. 5.1 correspond to $\alpha_{1}, \alpha_{2}$ and $\alpha_{3}$ in eq. 5.1. Note that the global variable freq is used in the weighting function, although freq has never been explicitly defined in the simulation setup. This works only because the MNS does not parse weighting functions before the simulation is submitted. Once the simulation has started the MNS computes the frequencies according to eq. 5.2.

\footnotetext{
${ }^{1}$ This applies for a current dependent current source. For other SDD the input spectrum may be a voltage, e.g., for a voltage dependent current source.
} 


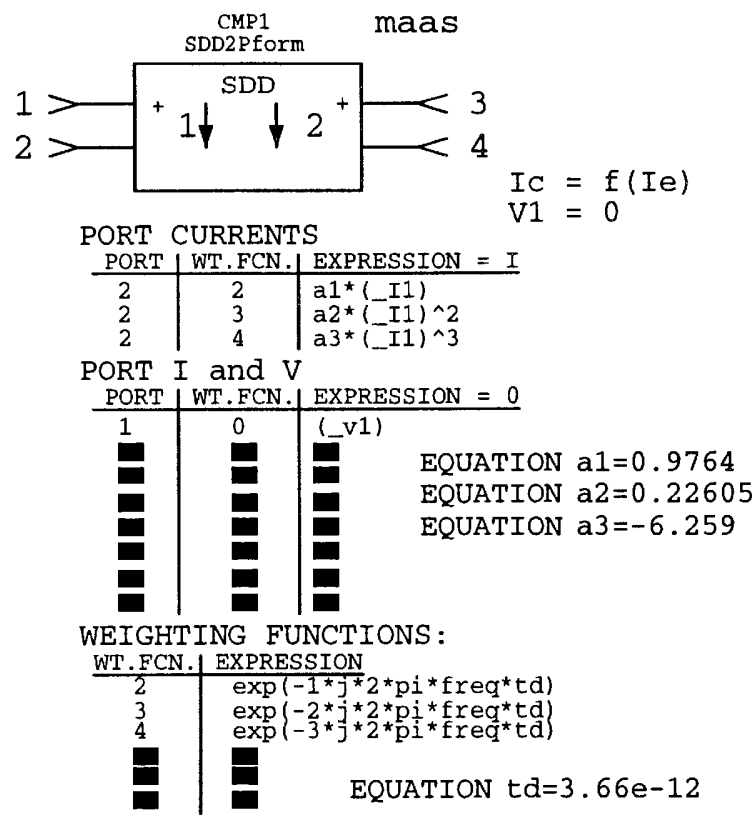

Figure 5.1: $\operatorname{SDD} I_{c}=f\left(I_{e}\right)$

\subsection{Weighting Function}

According to eq. 5.3 we need to find the factor (i.e., the weighting function) with which the spectrum is scaled. Applying the Fourier transform to eq. 5.1 yields

$$
\int_{-\infty}^{\infty} I_{c} \cdot e^{-j \omega t} d t=\int_{-\infty}^{\infty}\left(\alpha_{1} I_{e}\left(t-t_{d}\right)+\alpha_{2} I_{e}^{2}\left(t-t_{d}\right)+\alpha_{3} I_{e}^{3}\left(t-t_{d}\right)\right) e^{-j \omega t} d t
$$

Evaluating successively each term on the right side of eq. 5.4 leads to the single, double and triple convolution integral, respectively. 


\subsubsection{Convolution Integral}

The following expression is referred to as a single convolution integral:

$$
\int_{-\infty}^{\infty} \alpha_{1} I_{e}\left(t-t_{d}\right) \cdot e^{-j \omega t} d t
$$

Substituting $s=t-t_{d}$ leads to

$$
\int_{-\infty}^{\infty} \alpha_{1} I_{e}(s) \cdot e^{-j \omega\left(s+t_{d}\right)} d s=e^{-j \omega t_{d}} \int_{-\infty}^{\infty} \alpha_{1} I_{e}(s) \cdot e^{-j \omega s} d s=e^{-j \omega t_{d}} \cdot \tilde{I}_{\mathrm{c} 1}(\omega)
$$

The factor $e^{-j \omega t_{d}}$ is our weighting function and corresponds to weighting function 2 in Fig. 5.1.

\subsubsection{Double Convolution Integral}

The following expression is referred to as a double convolution integral:

$$
\int_{-\infty}^{\infty} \alpha_{2} I_{e}^{2}\left(t-t_{d}\right) \cdot e^{-j \omega t} d t=\int_{-\infty}^{\infty} \int_{-\infty}^{\infty} \alpha_{2} I_{e}\left(t-t_{d}\right) \cdot I_{e}\left(t-t_{d}\right) e^{-j \omega t, t} d t d t
$$

Substituting $s=t-t_{d}$ leads to

$$
\int_{-\infty}^{\infty} \int_{-\infty}^{\infty} \alpha_{2} I_{e}(s) \cdot I_{e}(s) e^{-j \omega\left(s+t_{d}\right)} e^{-j \omega\left(s+t_{d}\right)} d s d s=e^{-j 2 \omega t_{d}} \cdot \tilde{I}_{c 2}^{2}(\omega)
$$

The factor $e^{-j 2 \omega t_{d}}$ is our weighting function and corresponds to weighting function 3 in Fig. 5.1. The procedure for computing the weighting function for the triple convolution integral is analogous to the one in given here.

\subsection{Simulating the IP3}

Fig. 5.2 shows how the symbol (component 19) of the SDD is finally used in the HBT model. This Fig. corresponds to Fig. 5 in [6] and to Fig. 4.1 in section 4.3. It 
uses the fitted coefficients from [6] and is shown exactly as implemented in MDS.

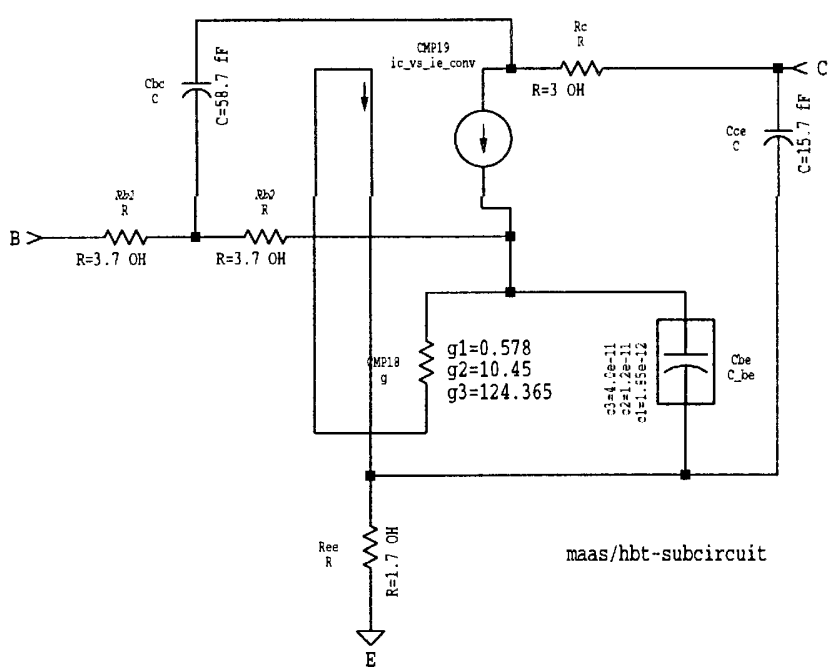

Figure 5.2: Circuit page showing the simulation setup in MDS 


\subsubsection{Implementing the Nonlinear Conductance}

Computing ${ }^{2}$ the first Taylor coefficient $g_{1}$ as given in section 4.6 , it follows:

$$
g_{1}=\frac{\partial I_{e}}{\partial V_{b e}}=\delta \cdot I_{0} e^{\delta V_{b e}} \approx \delta \cdot I_{e}
$$

if $e^{\delta V_{b e}} \gg 1$ which is the case for forward bias. Similiarly the second and third Taylor coefficient $g_{2}$ and $g_{3}$ give

$$
\begin{gathered}
g_{2}=\frac{\partial^{2} I_{e}}{\partial V_{b e}^{2}}=\frac{1}{2} \delta^{2} \cdot I_{0} e^{\delta V_{b e}} \approx \frac{1}{2} \delta^{2} \cdot I_{e} \\
g_{3}=\frac{\partial^{3} I_{e}}{\partial V_{b e}^{3}}=\frac{1}{6} \delta^{3} \cdot I_{0} e^{\delta V_{b e}} \approx \frac{1}{6} \delta^{3} \cdot I_{e}
\end{gathered}
$$

Since the bias point $V_{c e}=3 V, I_{c}=16 m A$ and $\alpha_{1}, \alpha_{2}, \alpha_{3}$ are given in [6], $I_{e}$ may be calculated from $I_{c}=\alpha_{1} I_{e}$. Fig. 5.3 shows the circuit page for this SDD representing the nonlinear conductance. Weighting function 0 is a built-in function and means multiplication by one. Fig. 5.4 shows the symbol page for this SDD as it appears, e.g., in Fig. 5.2, when this SDD is inserted as a subcircuit in a higher level circuit. Further note the way $g_{1}, g_{2}, g_{3}$ are specified here. In contrast to the SDD representing $I_{c}=f\left(I_{e}\right)$ the coefficients are specified on the so called scion page. In order to guide the MNS from the circuit page to the scion page, the coefficients $g_{1}, g_{2}, g_{3}$ need to be inserted as shown. This is done by executing the command INSERT/TEXT.

\footnotetext{
${ }^{2}$ The MAPLE computation for this is shown in appendix $C$. When excecuting the MAPLE worksheet, a comparison with eq. 5.9 - eq. 5.11 reveals disagreement among the coefficients. For example, the coefficients (based on Maas's [6] approximation) according to eq. 5.9 - eq. 5.11 are $g_{1}=0.586, g_{2}=10.45$ and $g_{3}=124.365$, respectively. The corresponding MAPLE coeeficients are $g_{1}=0.5503, g_{2}=9.8211$ and $g_{3}=116.85$, respectively. Only for small $\mathrm{v}$ the polynomials will be in agreement which again underlines the necessary limitaion of small excitation. In order to conform with Maas [6] intermodulation calculation the first set of coefficients has been used in the MDS simulation except $g_{1}$ has been adapted to reflect the fact that $g_{1}=1 / R_{j e}=0.571$. The average 0.578 has been used. The cancellation feature has been investigated using the MAPLE coefficients.
} 


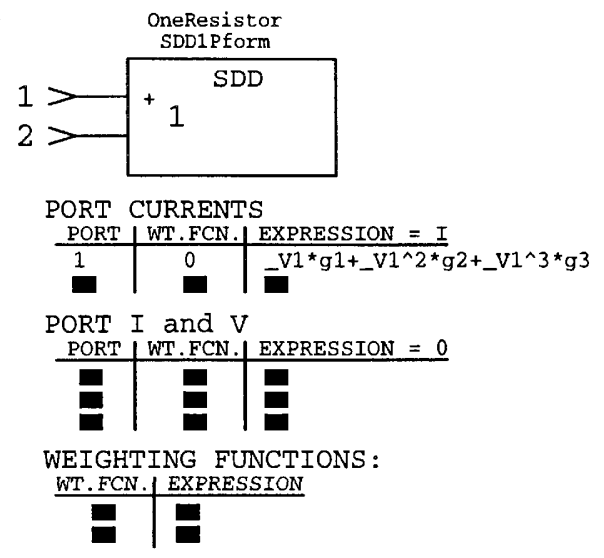

Figure 5.3: SDD - nonlinear resistor circuit page

Note that this is one of the rare occasions in MDS where typed text affects the simulation. Fig. 5.5 shows the scion page for this SDD. The scion page carries the $\underset{\text { QNAME }}{\text { QRABEL }} \begin{aligned} & \operatorname{ag} 1= \\ & \operatorname{ag} 2= \\ & \operatorname{ag} 3= \\ & 2\end{aligned}$

Figure 5.4: Symbol page

default values which are passed on to the circuit page if the MNS can not find values for the parameters specified on the circuit page and if the symbol page facilitates the transfer. 
$\begin{array}{llll}\text { Label } & g 1 & g 2 & g 3 \\ g & 0.578 & 10.45 & 124.365\end{array}$

Figure 5.5: Scion page

\subsubsection{Implementing the Nonlinear Capacitance}

Fig. 5.6 shows how eq. 4.18 from section 4.7 is implemented in the SDD. The port current is a voltage controlled expression which involves a time derivative. The time derivative corresponds to a multiplication with $j \omega$ in the frequency domain. The weighting function 1 performs this multiplication and is predefined in MDS.

\subsubsection{Final Simulation Setup}

Fig. 5.7 shows the final simulation setup. Oversample is set to 4 to decrease the aliasing error. Note, however, that this is done by selecting the box * TOI ANALYSIS * clicking on the command PERFORM/EDIT COMPONENT and then on the button MORE. The line oversample $=4$ is just a reminder and for convenience. It has no influence on the simulation. Tab.5.1 compares the IP3 computed by MDS according to Fig. 5.7 with Maas [6]. The simulation performs an input power level sweep. The IP3 is being compared for an input power level of $30 \mathrm{dBm}$. The 


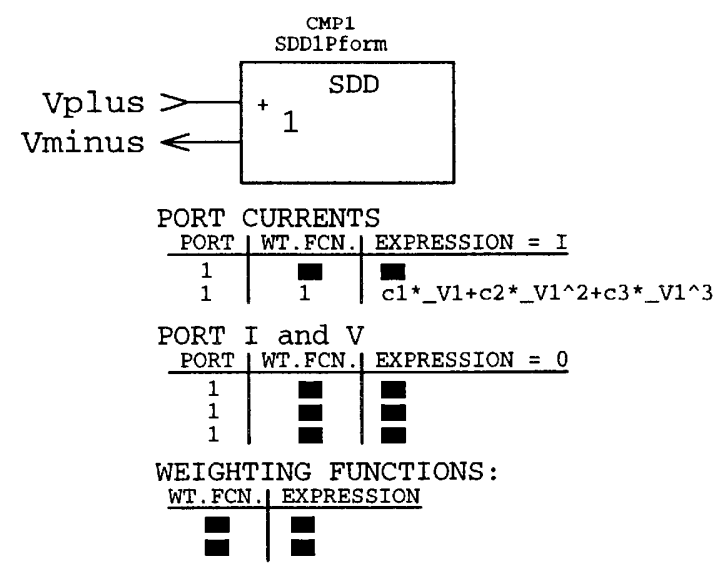

Figure 5.6: SDD - nonlinear capacitor

agreement is reasonable. It needs to be said, however, that Maas and Nelson did not publish the input power for their calculations and measurements, nor the frequency spacing between their two-tone excitation (for the MDS simulation the spacing was $100 \mathrm{MHz}$ ) and that the values shown in the table are read off from figures in [6]. All simulations used the $\alpha$ consisting of 3 components and time delay. Also, the table labels are a little bit cryptic. Here are the explanations:

- "MDS,g" is the simulation which employs the HBT model from Fig. 5.2

- "Maas,c" are the calculated values from [6] read off from Fig.4

- "Maas,m" are the measured values from [6] read off from Fig.4 


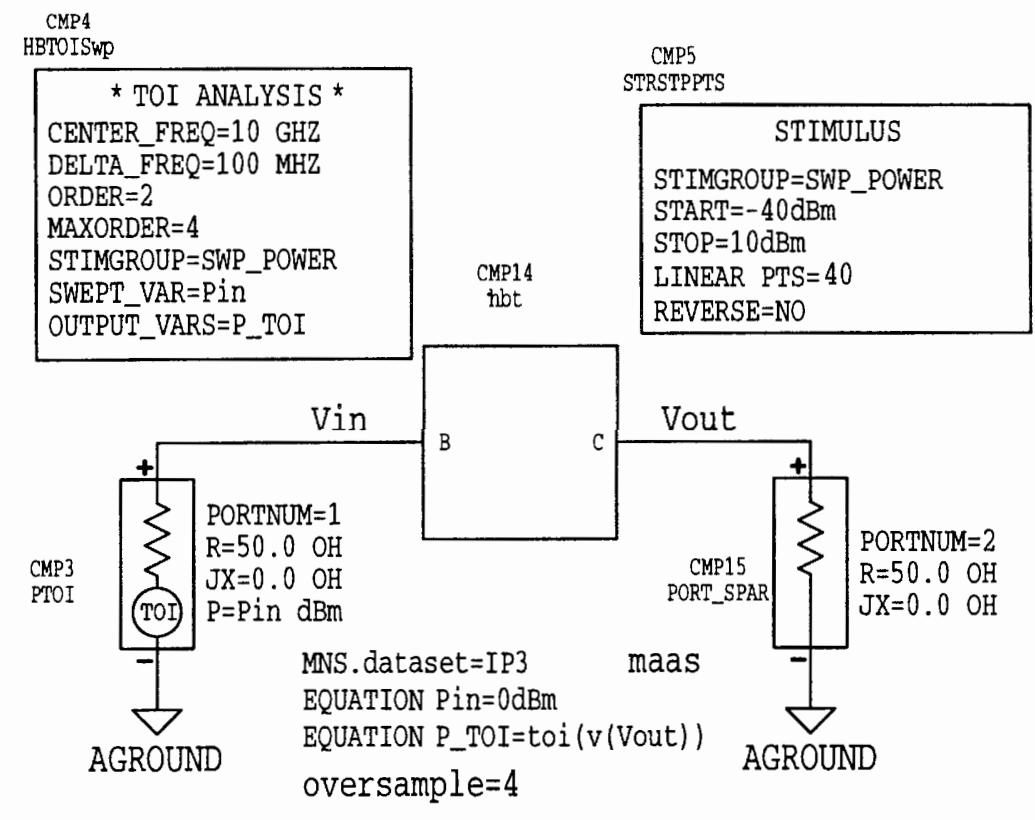

Figure 5.7: 2 tone HB simulation

- "MDS,tgc" employs the HBT model from Fig. 5.2 but the coefficients for the nonlinear conductance $\mathrm{g}$ and the nonlinear capacitor come from the Taylor series expansion in appendix C and D

- "MDS, dtc" same as "MDS,tgc" except the nonlinear conductance has been replaced by a diode

- the last column relates the work in this thesis to the IP3 calculation. This thesis focused on second order cancellation, but it is expected that the basic cancellation mechanism works for IP3 too. Hence this thesis laid the ground work for the practically important IP3 investigation 
Some of the columns in Tab.5.1 are shown again in Fig. 5.8 to illustrate the trend. It is clear that the pure Taylor coefficients (case 4) especially overemphasize the strength of the base emitter capacitance which was assumed to be diffusion dominated, resulting in much reduced IMD3 and hence higher IP3. The other graphs show good agreement with Maas' and Nelson's [6] experimental results.

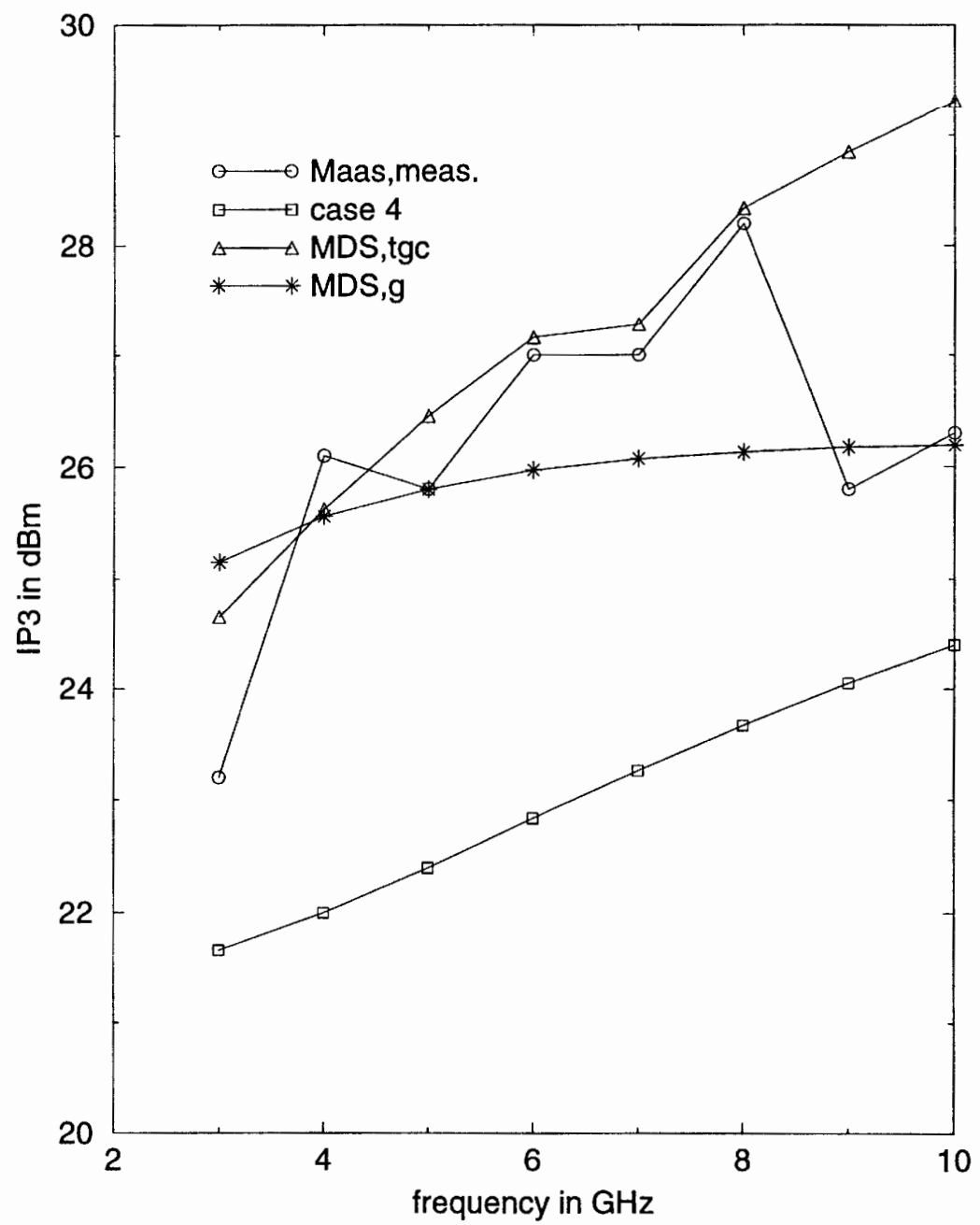

Figure 5.8: Various IP3 vs frequency 


\begin{tabular}{|l|l|l|l|l|l|l|}
\hline \hline $\mathrm{f}$ in GHz & MDS,g & Maas,c & Maas,m & MDS,tgc & MDS,dtc & case 4 \\
\hline 0.5 & 23.46 & & & 22.39 & 22.19 & 21.96 \\
\hline 0.7 & 23.49 & & & 22.29 & 22.17 & 21.87 \\
\hline 0.9 & 23.6 & & & 22.35 & 22.3 & 21.78 \\
\hline 1 & 23.67 & & & 22.41 & 22.4 & 21.74 \\
\hline 2 & 24.5 & 24 & & 23.51 & 23.71 & 21.5 \\
\hline 3 & 25.15 & 24.5 & 23.2 & 24.65 & 24.93 & 21.66 \\
\hline 4 & 25.56 & 25.1 & 26.1 & 25.62 & 24.94 & 22 \\
\hline 5 & 25.8 & 25.6 & 25.8 & 26.45 & 26.78 & 22.41 \\
\hline 6 & 25.97 & 26 & 27 & 27.16 & 27.51 & 22.84 \\
\hline 7 & 26.07 & 26.1 & 27 & 27.28 & 28.14 & 23.26 \\
\hline 8 & 26.13 & 26.2 & 28.2 & 28.34 & 28.7 & 23.67 \\
\hline 9 & 26.17 & 26.3 & 25.8 & 28.85 & 29.2 & 24.05 \\
\hline \hline
\end{tabular}

Table 5.1: Comparison of IP3 in $\mathrm{dBm}$ 


\section{Chapter 6}

\section{Conclusion}

A detailed investigation based on the equivalent circuit approach of the nonlinear behaviour of the base emitter junction in HBTs up to second order has been presented.

Simulation and analytical calculation of cancellation properties among nonlinear currents in the conductive part of the B-E junction were undertaken. Excellent agreement between the numerical tool (MDS) and the nonlinear current method implemented in the mathematics program MAPLE was obtained.

It is shown in detail how MDS simulates complex physical phenomena and how these phenomena are translated into mathematical expressions which can be solved by MDS, e.g. time delay entails convolution of spectra. A specific type of simulation called harmonic balance has been addressed generally and in terms of these phenomena. Executed worksheets for MAPLE are given in the appendix which may serve to double check simulation results.

The cancellation concept first introduced by Maas et al. [6] has been extended to an explicit treatment in terms of phase relationships among nodal and terminal 
voltages as well as currents up to second order. New analytical expressions were developed and tested numerically. Perfect agreement between the two was found. The derived analytical expressions not only establish a relationship between results reported by Wang et al. [5] and Maas et al. [6] but also extend the findings in [5]. Wang et al. [5] found that the emitter resistance does not affect the cancellation. This thesis suggests that $R_{e e}$ does have an influence on cancellation. The analytical expressions put the cancellation term " $c_{2} g_{1}-g_{2} c_{1}$ " which was originally found by Maas et al. [6] in a more general context by using this term to proove the equivalence of two different approaches to model the current gain in HBTs.

This thesis compared different approaches of modeling the HBT, showed their equivalence and highlighted the varoius approximations involved in each model at different stages and outlined their influence on IP2. The sensitivity of IP2 on various parameters has been shown, e.g. $\alpha$, its time delay, and $R_{e e}$.

The results reported here may serve as a starting point for future investigations of the practically more important issue of third order intermodulation (IMD3) and third order intercept point (IP3). By incorporating the time delay in the analytical expressions, deeper understanding of its influence on the cancellation mechanism may be gained. 


\subsection{Next Steps}

\subsubsection{Including the Base Collector Junction}

Investigating the nonlinearity in the base collector junction by the method outlined in this thesis leads to the interesting problem of juxtaposing these findings to Samelis' et al. [18] results ${ }^{1}$. This may lead to a more complete nonlinear model for HBTs.

Other trade offs may be looked into, e.g. $C_{b c}$ influences the high speed performance of a transistor according to $f_{T}=\frac{g_{m}}{2 \pi\left(C_{b c}+C_{b e}\right)}$. A reduction in $C_{b c}$ increases $f_{T}$ but it may also reduce the cancellation effect for the nonlinear currents [5]. The role of $C_{b c}$ is probably very interesting because it also presents the feedback of a transistor, hence its reduction increases device stability.

\subsubsection{More Physical Based Model of the Base Emitter Junc- tion}

The limitation of modeling the B-E capacitance as diffusion dominated has been shown. This calls for a more sophisticated approach to link equivalent circuit models to real device physics. It is believed that therein lies the key to future developments which do not require fitting parameters.

\footnotetext{
${ }^{1}$ They explored current cancellation at a so called base node. This is the node labeled as node 1 in Fig.4.21.
} 


\section{Appendix A}

\section{Decibels and nepers}

In Fig.2.1 the unit for power is $\mathrm{dBm}$. Here is a brief explanation [17]: Powers may be expressed in respect to some convenient reference power. If $P_{\text {ref }}=1 \mathrm{~mW}$ then a power level of $P=5 \mathrm{~mW}$ converted into $\mathrm{dBm}$ looks like this:

$$
P_{d B m}=10 \cdot \log \frac{P}{P_{\text {ref }}}=10 \cdot \log \frac{5 m W}{1 m W} \approx 7
$$

Vice versa it looks like this:

$$
P_{m W}=10^{\frac{P_{d B m}}{10}}=10^{0.7} \approx 5
$$

In the literature $\mathrm{dBm}$ is usually used instead of the complete expression $\mathrm{dBmW}$. If no reference power is specified $1 \mathrm{~mW}$ is assumed. Just for the sake of completeness, the power gain may be expressed in $\mathrm{dB}$ as follows:

$$
G_{d B}=10 \cdot \log \frac{P_{\text {out }}}{P_{\text {in }}}
$$

and in nepers $(\mathrm{Np})$ as:

$$
G_{N p}=\frac{1}{2} \cdot \ln \frac{P_{\text {out }}}{P_{\text {in }}}
$$


The conversion between nepers and decibels is: $1 N p=8.686 \mathrm{~dB}$. The relation between voltage and power gain may be established as follows. Power gain may be computed from voltage gain, input, and load impedance, which for simplicity are assumed to be resistive:

$$
G=A_{v}^{2} \cdot \frac{R_{\text {in }}}{R_{\text {load }}}
$$

which looks in $\mathrm{dB}$ like:

$$
G_{d B}=10 \log A_{v}^{2}+10 \log R_{\text {in }}-10 \log R_{\text {load }}=20 \log A_{v}+10 \log \frac{R_{\text {in }}}{R_{\text {load }}}
$$

It is kind of obvious from Eq.A.6 to define the voltage gain like:

$$
A_{v d B}=20 \cdot \log \left|A_{v}\right|
$$

It is not that obvious to define the voltage gain now in nepers which is nevertheless done:

$$
A_{v N p}=\ln \frac{V_{\text {out }}}{V_{\text {in }}}
$$




\title{
Appendix B
}

\section{MAPLE Calculation of}

\author{
$\left(V_{x} \cos m \omega_{x} t+\cdots\right)^{n}$
}

What follows is the pure MAPLE input!

$>i:=g 1 * v+g 2 * v^{-} 2+g 3 * v^{-} 3$;

$i:=g 1 v+g 2 v^{2}+g 3 v^{3}$

$>\mathrm{v}:=\mathrm{v} 1+\mathrm{v} 2+\mathrm{v} 3$

$v:=v 1+v \mathscr{2}+v 3$

> v1:=Va*cos (omega*t+alpha);

$v 1:=V a \cos (\omega t+\alpha)$

$>\mathrm{v} 2:=\mathrm{Vb} * \cos (2 *$ omega $* t+$ beta $)$;

$v \mathscr{2}:=V b \cos (2 \omega t+\beta)$

$>$ v3:=Vc*cos $(3 *$ omega $* t+$ gamma $)$;

v3 $:=V c \cos (3 \omega t+\gamma)$ 
> ii:=simplify(i, trig);

$$
\begin{aligned}
i i:= & g 1 V a \% 3+g 1 V b \% 2+g 1 V c \% 1+g 2 V a^{2} \% 3^{2} \\
& +2 g 2 V a \% 3 V b \% 2+2 g 2 V a \% 3 V c \% 1+g 2 V b^{2} \% 2^{2} \\
& +2 g 2 V b \% 2 V c \% 1+g 2 V c^{2} \% 1^{2}+g 3 V a^{3} \% 3^{3} \\
& +3 g 3 V a^{2} \% 3^{2} V b \% 2+3 g 3 V a^{2} \% 3^{2} V c \% 1 \\
& +3 g 3 V a \% 3 V b^{2} \% 2^{2}+6 g 3 V a \% 3 V b \% 2 V c \% 1 \\
& +3 g 3 V a \% 3 V c^{2} \% 1^{2}+g 3 V b^{3} \% 2^{3}+3 g 3 V b^{2} \% 2^{2} V c \% 1 \\
& +3 g 3 V b \% 2 V c^{2} \% 1^{2}+g 3 V c^{3} \% 1^{3} \\
& \% 1:=\cos (3 \omega t+\gamma) \\
& \% 2:=\cos (2 \omega t+\beta) \\
& \% 3:=\cos (\omega t+\alpha)
\end{aligned}
$$

> iii:=combine(ii, trig);

$$
\begin{aligned}
i i i: & =\frac{3}{2} g 3 V b \% 2 V c^{2}+\frac{3}{4} g 3 V b^{2} V c \cos (\omega t-\gamma+2 \beta) \\
& +\frac{3}{4} g 3 V b^{2} V c \cos (7 \omega t+\gamma+2 \beta)+\frac{1}{4} g 3 V c^{3} \cos (9 \omega t+3 \gamma) \\
& +\frac{3}{4} g 3 V c^{3} \% 1+\frac{3}{4} g 3 V b V c^{2} \cos (4 \omega t-\beta+2 \gamma) \\
& +\frac{3}{4} g 3 V b V c^{2} \cos (8 \omega t+\beta+2 \gamma)+\frac{3}{2} g 3 V a \% 3 V b^{2} \\
& +\frac{3}{4} g 3 V a V c^{2} \cos (-5 \omega t+\alpha-2 \gamma)+g 1 V a \% 3+g 1 V b \% 2 \\
& +g 1 V c \% 1+\frac{3}{2} g 3 V a \% 3 V c^{2} \\
& +\frac{3}{2} g 3 V a V b V c \cos (-4 \omega t-\gamma+\alpha-\beta) \\
& +\frac{3}{2} g 3 V a V b V c \cos (2 \omega t+\gamma+\alpha-\beta) \\
& +\frac{3}{2} g 3 V a V b V c \cos (-\gamma+\alpha+\beta) \\
& +\frac{3}{2} g 3 V a V b V c \cos (6 \omega t+\gamma+\alpha+\beta) \\
& +\frac{3}{4} g 3 V a V b^{2} \cos (-3 \omega t+\alpha-2 \beta)+\frac{1}{2} g 2 V a^{2}
\end{aligned}
$$


$+\frac{1}{2} g 2 V a^{2} \cos (2 \omega t+2 \alpha)+\frac{3}{4} g 3 V a V b^{2} \cos (5 \omega t+\alpha+2 \beta)$

$+\frac{3}{4} g 3 V a V c^{2} \cos (7 \omega t+\alpha+2 \gamma)+\frac{1}{2} g 2 V b^{2}$

$+\frac{1}{2} g 2 V^{2} \cos (4 \omega t+2 \beta)+g 2 V a V b \cos (-\omega t+\alpha-\beta)$

$+g 2 V a V b \cos (3 \omega t+\alpha+\beta)+g 2 V a V c \cos (-2 \omega t+\alpha-\gamma)$

$+g 2 V a V c \cos (4 \omega t+\alpha+\gamma)+\frac{1}{2} g 2 V c^{2}$

$+\frac{1}{2} g \mathscr{2} V c^{2} \cos (6 \omega t+2 \gamma)+g 2 V b V c \cos (\omega t-\beta+\gamma)$

$+g^{2} V b V c \cos (5 \omega t+\beta+\gamma)+\frac{3}{2} g 3 V b^{2} V c \% 1$

$+\frac{1}{4} g 3 V a^{3} \cos (3 \omega t+3 \alpha)+\frac{3}{4} g 3 V a^{3} \% 3+\frac{3}{2} g 3 V a^{2} V b \% 2$

$+\frac{1}{4} g 3 V b^{3} \cos (6 \omega t+3 \beta)+\frac{3}{4} g 3 V b^{3} \% 2$

$+\frac{3}{4} g 3 V a^{2} V b \cos (-\beta+2 \alpha)+\frac{3}{4} g 3 V a^{2} V b \cos (4 \omega t+\beta+2 \alpha)$

$+\frac{3}{2} g 3 V a^{2} V c \% 1+\frac{3}{4} g 3 V a^{2} V c \cos (-\omega t-\gamma+2 \alpha)$

$+\frac{3}{4} g 3 V a^{2} V c \cos (5 \omega t+\gamma+2 \alpha)$

$\% 1:=\cos (3 \omega t+\gamma)$

$\% 2:=\cos (2 \omega t+\beta)$

$\% 3:=\cos (\omega t+\alpha)$ 


\section{Appendix C}

\section{MAPLE Taylor Series Expansion}

\section{of a Nonlinear Conductance}

MAPLE expansion of the diode equation and executed MAPLE worksheet, which provides insight when the small signal voltage amplitude becomes too large. Focus on the line in the executed worksheet:

$$
i=0.015+0.55 v+9.82 v^{2}+116.85 v^{3}
$$

As $\mathrm{v}$ contains (among other terms) a term $V \cos \omega t$, the quadratic term generates a dc component according to $(V \cos \omega t)^{2}=0.5 V^{2}(1+\cos 2 \omega t)$. The question is which amplitude $\mathrm{V}$ will bring the generated $\mathrm{dc}$ component in the range of the original $\mathrm{dc}$ bias point i.e. $I_{e}=\alpha_{1} I_{c}=0.9764 \cdot 16 m A=0.015$.

$$
0.015=\frac{9.82}{2} V^{2}
$$

Thus an amplitude of about $55 \mathrm{mV}$ will significantly offset the bias point. Begin MAPLE input: 
$>$ Ie: $=$ Io* $\left(\exp \left(\operatorname{delta} *\left(\mathrm{Vbe}_{\mathrm{b}}+\mathrm{v}\right)\right)-1\right)$;

$I e:=I o\left(\mathrm{e}^{(\delta(V b e+v))}-1\right)$

$>$ ie: $=\operatorname{series}(\mathrm{Ie}, \mathrm{v}, 4)$;

$i e:=I o\left(\mathrm{e}^{(\delta V b e)}-1\right)+I o \mathrm{e}^{(\delta V b e)} \delta v+\frac{1}{2} I o \mathrm{e}^{(\delta V b e)} \delta^{2} v^{2}+\frac{1}{6} I o \mathrm{e}^{(\delta V b e)} \delta^{3}$ $v^{3}+\mathrm{O}\left(v^{4}\right)$

$>$ type(ie,polynom);

false

$>i:=$ convert(ie,polynom);

$i:=I o\left(\mathrm{e}^{(\delta V b e)}-1\right)+I o \mathrm{e}^{(\delta V b e)} \delta v+\frac{1}{2} I o \mathrm{e}^{(\delta V b e)} \delta^{2} v^{2}+\frac{1}{6} I o \mathrm{e}^{(\delta V b e)} \delta^{3} v^{3}$

$>$ type $(i, p o l y n o m($ anything,v));

true

$>$ Io:=1e-23;

Io $:=.110^{-\mathbf{2 2}}$

$>$ delta:=charge/(eta*boltzmann*temp);

$\delta:=\frac{\text { charge }}{\eta \text { boltzmann temp }}$

$>$ temp:=305;

temp $:=305$

$>$ boltzmann: $=1.38 \mathrm{e}-23$;

boltzmann $:=.13810^{-22}$

$>$ charge:=1.6e-19;

charge $:=.1610^{-18}$ 


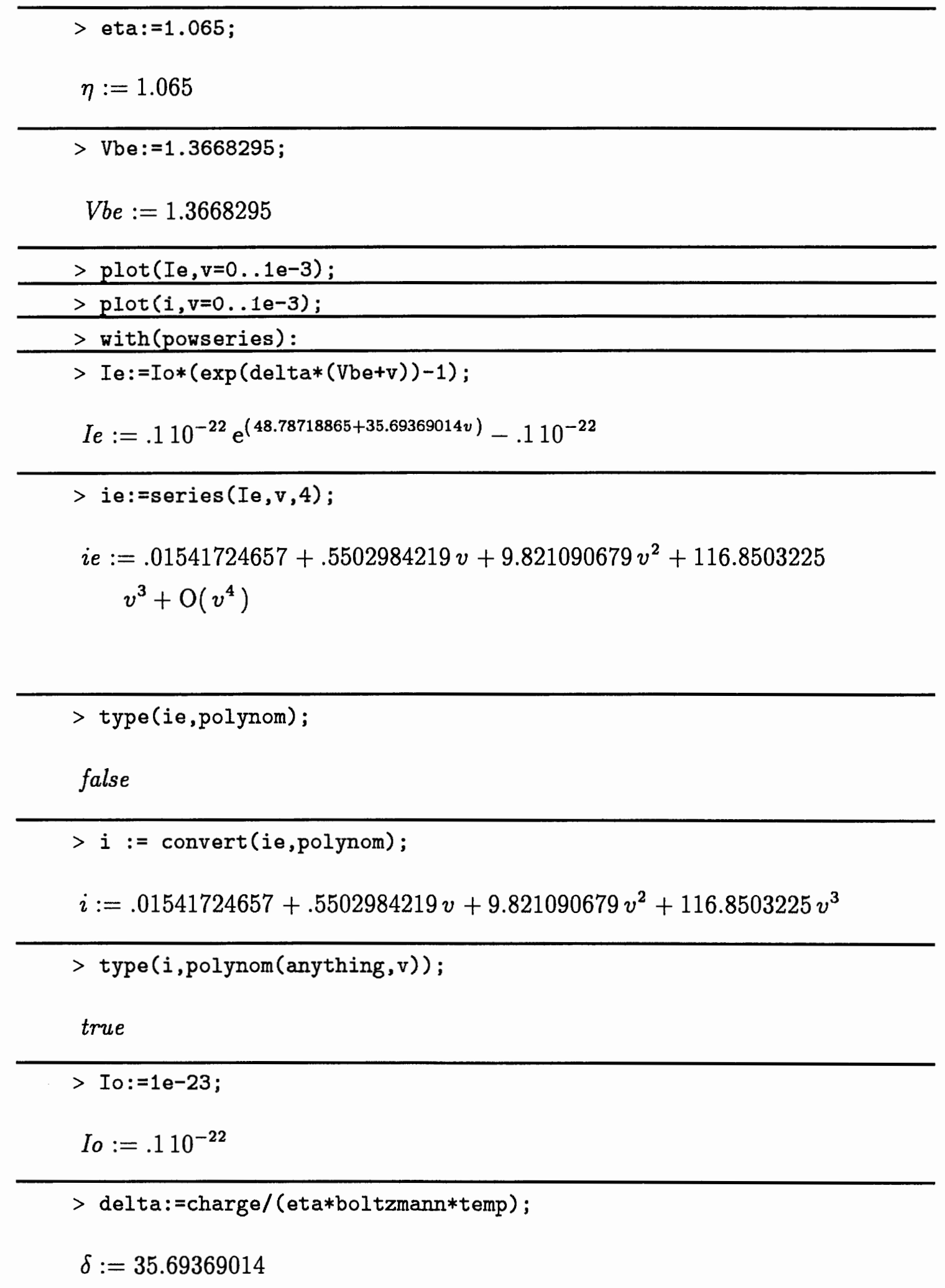




\begin{tabular}{l}
$>$ temp $:=305 ;$ \\
temp $:=305$ \\
\hline boltzmann $:=1.38 \mathrm{e}-23 ;$ \\
boltzmann $:=.13810^{-22}$ \\
\hline charge $:=1.6 \mathrm{e}^{-19 ;}$ \\
charge $:=.1610^{-18}$ \\
$>$ eta $:=1.065 ;$ \\
$\eta:=1.065$ \\
$>$ Vbe $:=1.3668295 ;$ \\
Vbe $:=1.3668295$ \\
\hline$>$ plot $($ Ie, $\mathrm{v}=0 . .1 \mathrm{e}-3) ;$
\end{tabular}




\section{Appendix D}

\section{MAPLE Taylor Series Expansion}

\section{of Nonlinear Capacitance}

$>$ with(powseries):

$>$ Q:=tau*Io* $\left(\exp \left(\operatorname{delta} *\left(\mathrm{Vbe}^{+}+\mathrm{v}\right)\right)-1\right)$;

$Q:=\tau I o\left(\mathrm{e}^{(\delta(V b e+v))}-1\right)$

$>q:=\operatorname{series}(Q, v, 4)$;

$$
\begin{aligned}
q:= & \tau I o\left(\mathrm{e}^{(\delta V b e)}-1\right)+\tau I o \mathrm{e}^{(\delta V b e)} \delta v+\frac{1}{2} \tau I o \mathrm{e}^{(\delta V b e)} \delta^{2} v^{2}+ \\
& \frac{1}{6} \tau I o \mathrm{e}^{(\delta V b e)} \delta^{3} v^{3}+\mathrm{O}\left(v^{4}\right)
\end{aligned}
$$

$>$ type $(q$, polynom $)$;

false

$>p:=\operatorname{convert}(q$, polynom $)$

$$
\begin{aligned}
p:= & \tau I o\left(\mathrm{e}^{(\delta V b e)}-1\right)+\tau I o \mathrm{e}^{(\delta V b e)} \delta v+\frac{1}{2} \tau I o \mathrm{e}^{(\delta V b e)} \delta^{2} v^{2} \\
& +\frac{1}{6} \tau I o \mathrm{e}^{(\delta V b e)} \delta^{3} v^{3}
\end{aligned}
$$




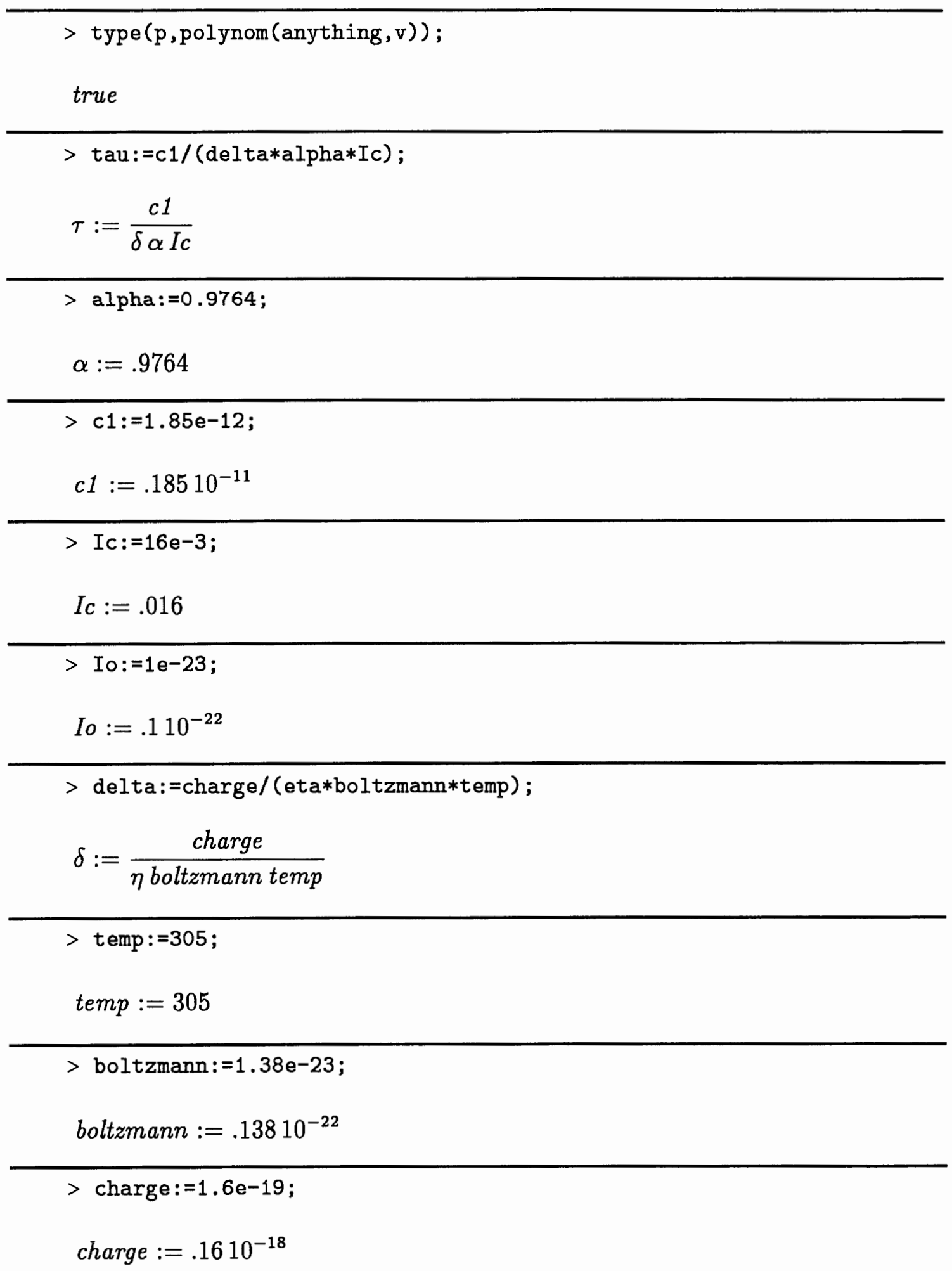


$>$ eta $:=1.065$;

$\eta:=1.065$

$>$ Vbe: $=1.3668295$;

Vbe $:=1.3668295$

$>$ with(powseries):

$>\mathrm{Q}:=\operatorname{tau} * \operatorname{Io} *(\exp (\operatorname{delta} *(\operatorname{Vbe}+\mathrm{v}))-1)$;

$Q:=.331766495910^{-34} \mathrm{e}^{(48.78718865+35.69369014 v)}-.331766495910^{-34}$

$>\mathrm{q}:=\operatorname{series}(\mathrm{Q}, \mathrm{v}, 4)$;

$q:=.511492587110^{-13}+.182570579110^{-11} v+.325830884010^{-10} v^{2}+$ $.387670220410^{-9} v^{3}+\mathrm{O}\left(v^{4}\right)$

$>\operatorname{type}(q$, polynom $)$;

false

$>p:=\operatorname{convert}(q$, polynom $) ;$

$p:=.511492587110^{-13}+.182570579110^{-11} v+.325830884010^{-10} v^{2}$ $+.387670220410^{-9} v^{3}$

$>\operatorname{type}(p, p o l y n o m(a n y t h i n g, v))$;

true

$>$ tau:=c1/(delta*alpha*Ic);

$\tau:=.331766495910^{-11}$

$>$ alpha:=0.9764;

$\alpha:=.9764$ 


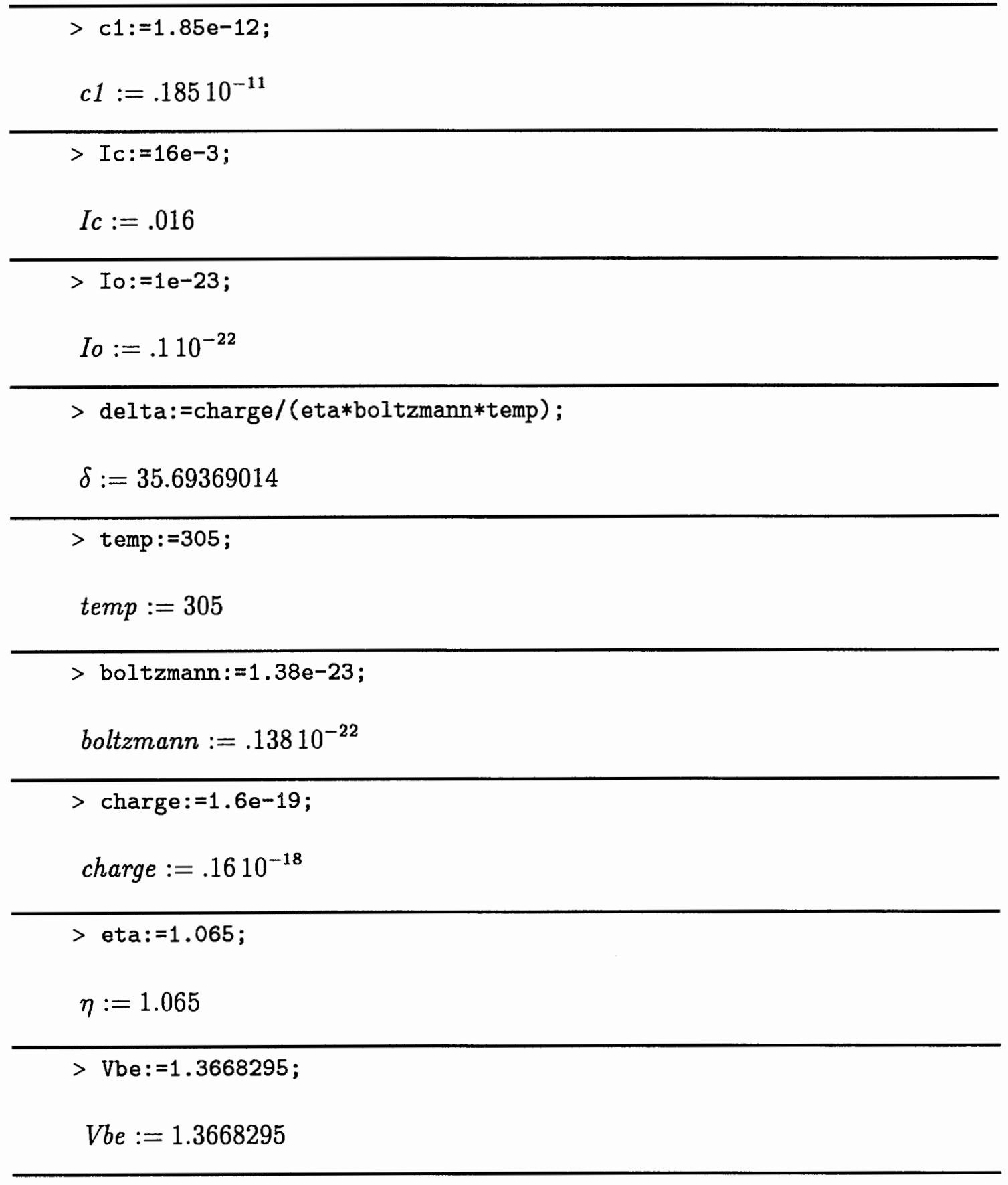




\section{Appendix E}

\section{MAPLE Verification of Phase}

\section{Difference}

$$
\begin{aligned}
& >\mathrm{I} 1:=\mathrm{Vs} 1 / \mathrm{Z} ; \\
& I 1:=.001 \frac{1}{7.4-2.738666890 \frac{I}{\pi}}
\end{aligned}
$$

\begin{tabular}{l}
$>\mathrm{Vs} 1:=1 \mathrm{e}-3 * \exp (\mathrm{I} * 0) ;$ \\
$V s 1:=.001$ \\
\hline$>c 1:=0.1825705791 \mathrm{e}-11 ;$ \\
$c 1:=.182570579110^{-11}$ \\
\hline$>\mathrm{R}:=7.4 ;$ \\
$R:=7.4$ \\
$>$ omega $:=2 * \mathrm{Pi} * \mathrm{f} ;$ \\
$\omega:=.20010^{12} \pi$ \\
$>\mathrm{f}:=100 \mathrm{e} 9 ;$ \\
$f:=.10010^{12}$
\end{tabular}


$>\mathrm{Z}:=\mathrm{R}+1 /(\mathrm{I} *$ omega*c1)

$$
Z:=7.4-2.738666890 \frac{I}{\pi}
$$

$>$ I1p:=convert $(I 1$, polar $)$;

$$
\begin{gathered}
I 1 p:=\operatorname{polar}\left(.001 \frac{1}{\sqrt{54.76+7.500296334 \frac{1}{\pi^{2}}}},\right. \\
\left.\quad \text { argument }\left(\frac{1}{7.4-2.738666890 \frac{I}{\pi}}\right)\right)
\end{gathered}
$$

$>$ I1m:=map (evalc, ");

$$
\operatorname{polar}\left(.001 \frac{1}{\sqrt{54.76+7.500296334 \frac{1}{\pi^{2}}}}, \arctan \left(.3700901203 \frac{1}{\pi}\right)\right)
$$

$>$ I1c1:=simplify $(\mathrm{I} 1 \mathrm{~m})$;

$I 1 c 1:=\operatorname{polar}(.0001342071056, .1172628934)$

$>\mathrm{V} 1:=\mathrm{I} 1 \mathrm{c} 1 /(\mathrm{I} *$ omega*c1);

$V 1:=-2.738666890 \frac{I \operatorname{polar}(.0001342071056, .1172628934)}{\pi}$

$>\mathrm{V} 1 \mathrm{c}:=\operatorname{convert}(\mathrm{V} 1$, polar $)$;

$V 1 c:=\operatorname{polar}(.0001169943392,-1.453533433)$

$>\mathrm{I} 2:=\mathrm{I} *$ omega $* \mathrm{c} 2 * \mathrm{~V} 1 \mathrm{c}^{\wedge} 2$;

I2 $:=6.516617680 I \pi$ polar $(.0001169943392,-1.453533433)^{2}$ 


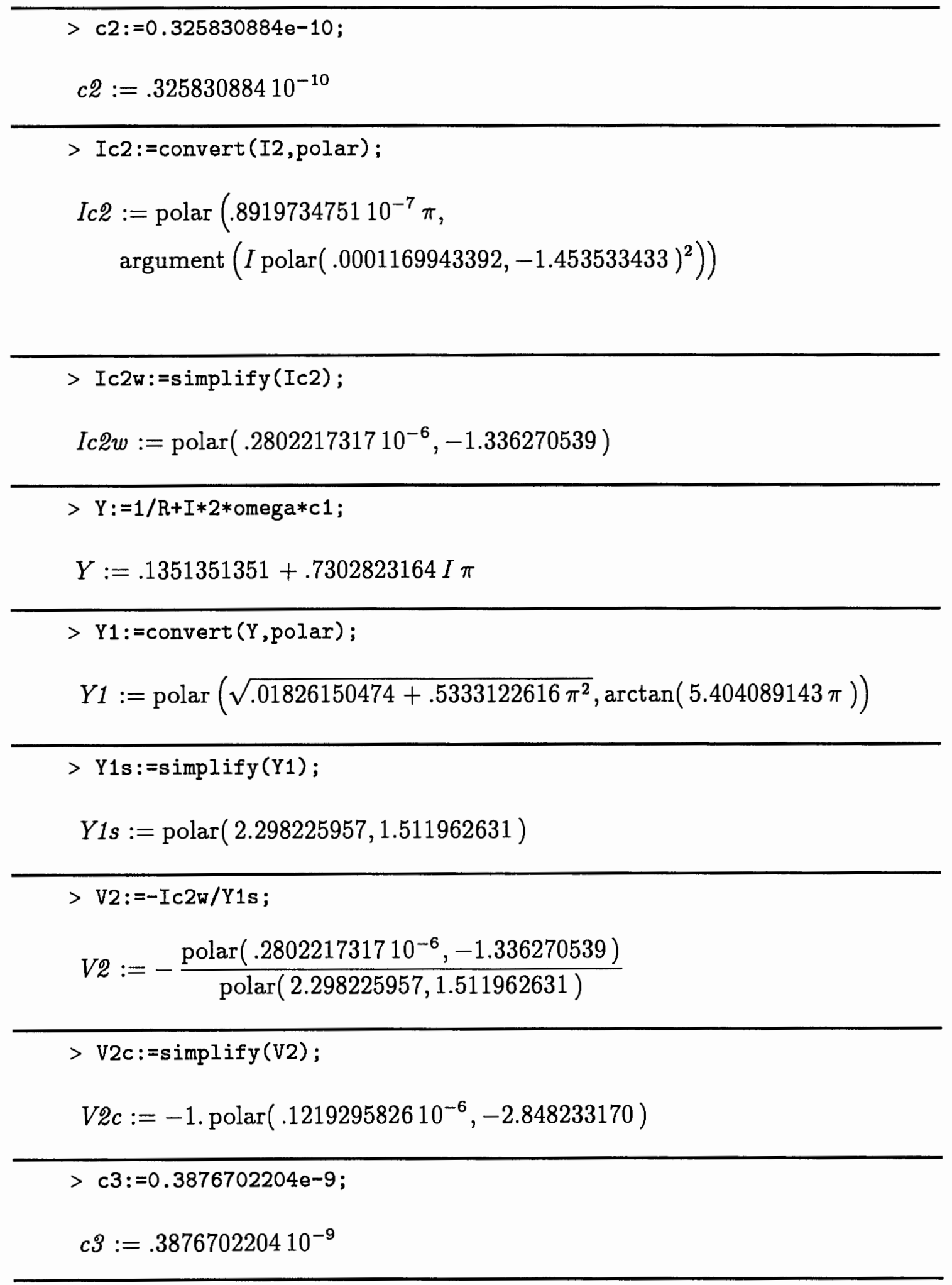




\section{Appendix $\mathbf{F}$}

\section{MAPLE Nonlinear Current}

\section{Method Case 5}

\begin{tabular}{l}
$>\mathrm{Vs}:=0.001 ;$ \\
$V s:=.001$ \\
\hline$>\mathrm{Rb}:=7.4 ;$ \\
$R b:=7.4$ \\
\hline$>\mathrm{f}:=10 * 1 \mathrm{e} 9 ;$ \\
$f:=.1010^{11}$ \\
$>$ Ree $:=1.7 ;$ \\
$R e e:=1.7$ \\
$>\mathrm{c} 1:=0.1825705791 \mathrm{e}-11 ;$ \\
$c 1:=.182570579110^{-11}$ \\
$>\mathrm{g} 1:=0.5502984219 ;$ \\
$g 1:=.5502984219$ \\
$>$ alpha $:=0.9764 ;$ \\
$\alpha 0:=.9764$
\end{tabular}




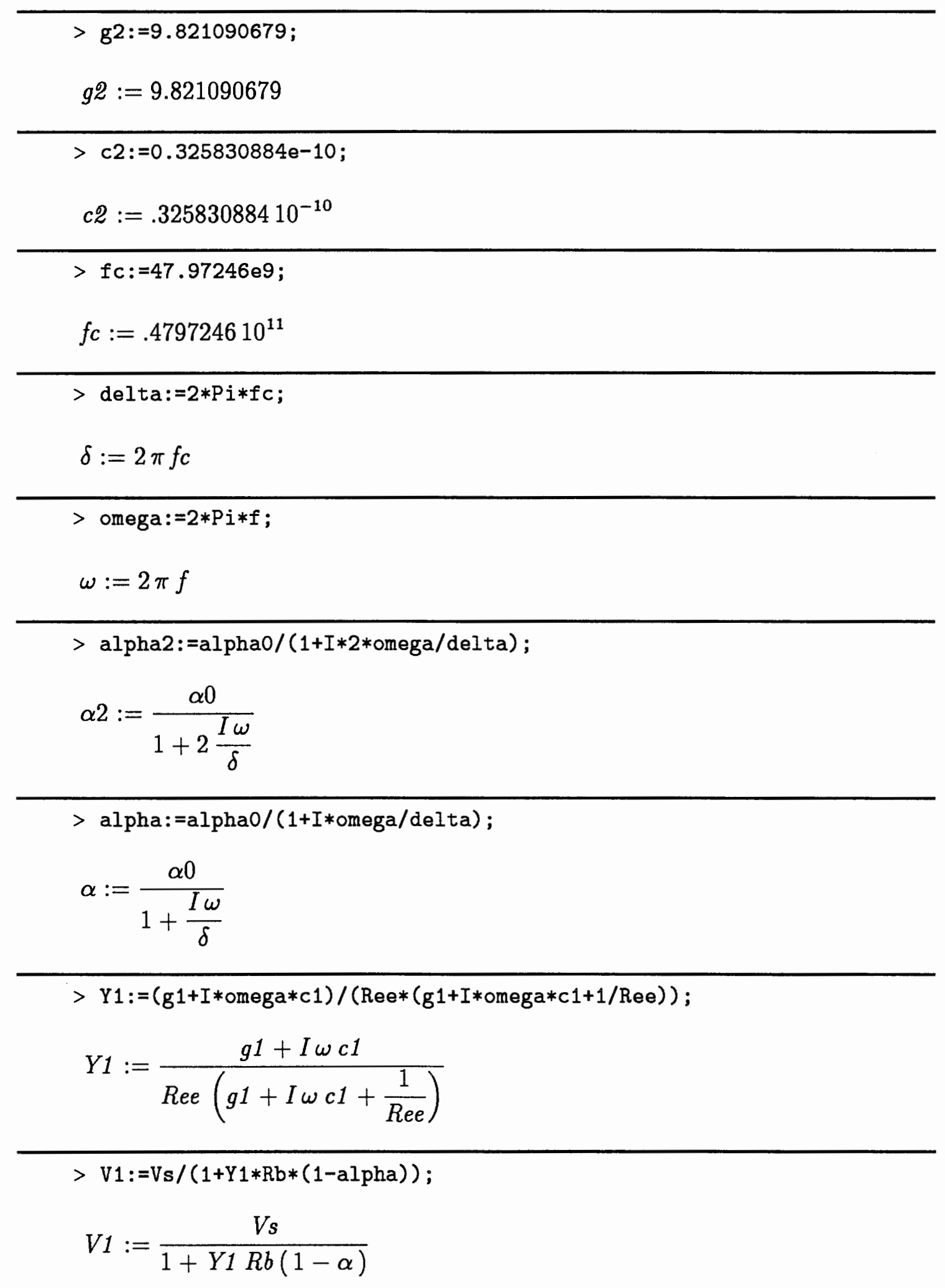


$>$ V1s:=simplify $(\mathrm{V} 1)$;

$V 1 s:=.0007927299870-.0003113295854 I$

$>\operatorname{V1ree}:=\mathrm{V} 1 \mathrm{~s} * \operatorname{Ree} /(\operatorname{Ree}+(1 /(\mathrm{g} 1 * \mathrm{I} *$ omega*c1 $) /(1 / \mathrm{g} 1+1 /(\mathrm{I} *$ omega*c1 $))))$;

$$
\text { V1ree }:=\frac{\text { V1s Ree }}{\text { Ree }-\frac{I}{g 1 \omega c 1\left(\frac{1}{g 1}-\frac{I}{\omega c 1}\right)}}
$$

$>$ V1rees:=simplify (V1ree);

V1rees $:=.0004033174451-.0001112428206 I$

$>$ V1term: $=$ V1s-V1rees;

V1term $:=V 1 s-$ V1rees

$>$ pV1term:=argument (V1term);

$p$ V1term $:=-.4746399719$

$>\operatorname{Ig} 2:=0.5 * g 2 *$ V $\operatorname{term}^{-} 2$;

Ig2 $:=.5 \mathrm{~g}^{2} \mathrm{~V}$ term $^{2}$

$>$ Ic2:=I*omega*c2*V1term^2;

$I c \mathscr{Q}:=I \omega c \mathscr{2}$ V1term ${ }^{2}$

> V2:=I*(alpha $2 * \mathrm{Ic} 2 * \mathrm{Rb}-\mathrm{Ic} 2 * \mathrm{Rb}+\mathrm{alpha} 2 * \mathrm{Ig} 2 * \mathrm{Rb}-\mathrm{Ig} 2 * \mathrm{Rb}) /$

$>(-\mathrm{I} * \mathrm{Rb} * \mathrm{alpha} 2 * \mathrm{~g} 1+2 * \mathrm{Rb} * \mathrm{alpha} 2 * \mathrm{c} 1 *$ omega $-2 *$

$>\mathrm{Rb} * \mathrm{c} 1 *$ omega $-2 * \mathrm{Ree} * c 1 *$ omega $+\mathrm{I} * \mathrm{~g} 1 * \mathrm{Rb}+\mathrm{I}+\mathrm{I} * \mathrm{~g} 1 * \mathrm{Ree})$;

$V 2:=I(\alpha 2 I c \mathscr{2} R b-I c 2 R b+\alpha 2 I g 2 R b-I g 2 R b) /(-I R b \alpha 2 g 1$ $+2 R b \alpha 2 c 1 \omega-2 R b c 1 \omega-2$ Ree $c 1 \omega+I g 1 R b+I+I g 1 R e e$ ) 


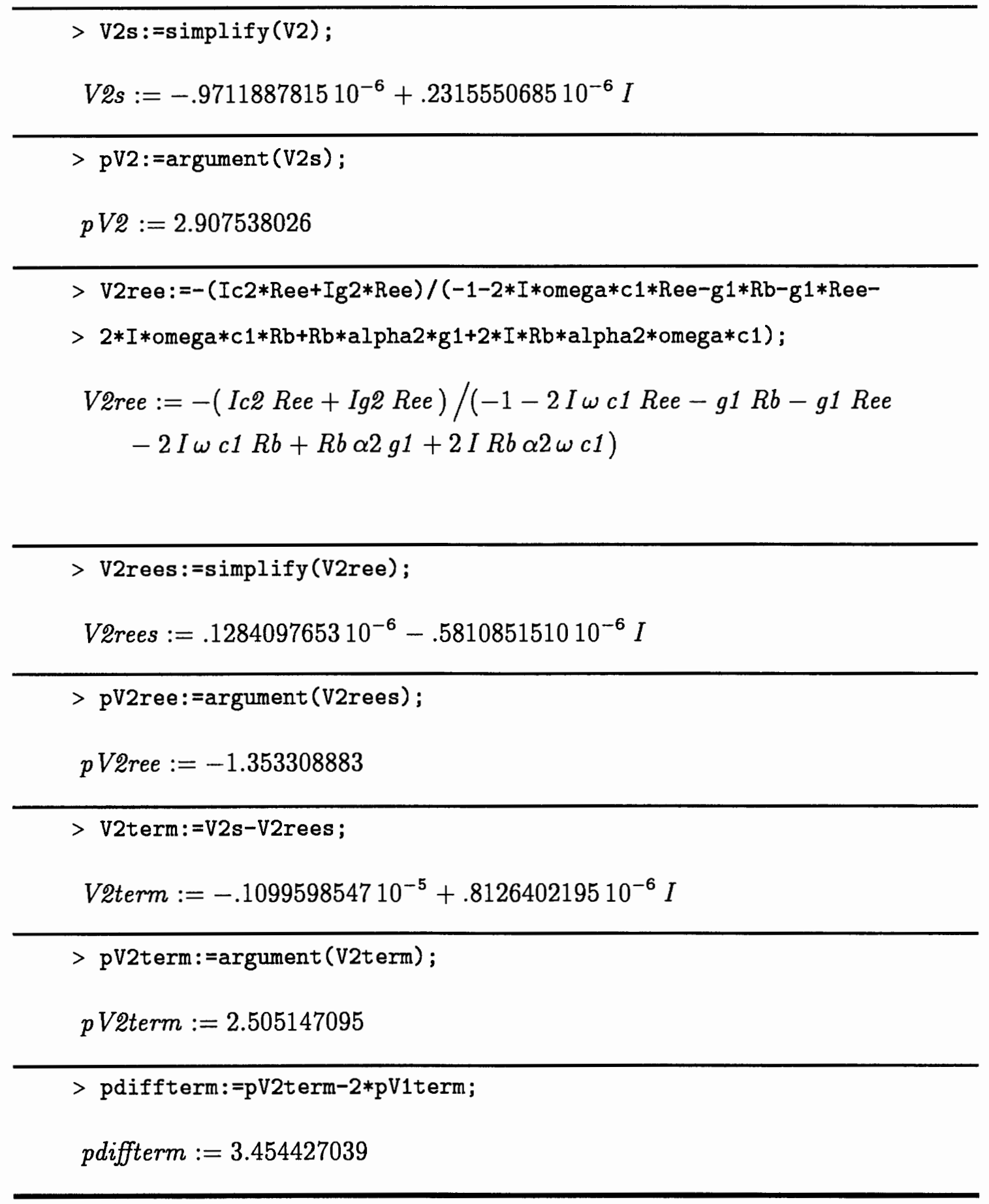




\section{Appendix G}

\section{Time Waveform in MDS}

The purpose of this appendix is twofold:

- a sufficient number of harmonics is needed to represent the time waveform correctly.

- it compares the two methods in MDS to create waveforms for the harmonics.

Fig. G.1 resembles Fig. 4.7 but this time 8 harmonics have been considered and the "IF - THEN" condition has been used. The higher number of harmonics approximates the waveform closer which can be seen by comparing vecall=ts(vec) from Fig. G.1 with $v e b=t s(v e c)$ from Fig. 4.7. The function " $t s$ " creates the waveform in the time domain of a spectrum at a given node (in this case the node is labeled vec, see Fig. 4.6). Thus, these two equations on a presentation icon's DISPLAY PAGE in MDS give the true waveform as far as the accuracy of the simulation goes.

Now there are two methods to analyze this full frequency waveform further. In Fig. 4.7 the function "generate" has been used. Let's take a closer look at the 
equation

$$
t 3=\text { generate }(0,2 * 3 / e q 1[4], 201)
$$

Eq. G.1 will produce the independent variable which in time domain is, of course, the time $t$ for the waveform. The label " $t 3$ " is arbitrary but it alludes already to the third harmonic. The first number in the argument of the function "generate" determines the start value which is zero here. The second number gives the stop value. By default, MDS considers 2 periods for the fundamental waveform. Accordingly, the third harmonic will have 6 periods within this intervall which is expressed by $2 * 3 /$ eq1[4]. The expression "eq1[4]" selects the fourth entry of the dataset array specified by Eq1=rc.IVARDATA1.freq which corresponds to the third harmonic (the first entry is DC, therefore the fourth is the third harmonic). Recall that $1 / f=T$. Eq1=rc.IVARDATA1.freq is an equation inserted from DATASET which is done by clicking on ID on the DISPLAY PAGE. The last number determines the number of points, here 201. The equation

$$
v e b 3=\operatorname{mag}(e q 2[4]) * \cos (2 * P i * e q 1[4] * t 3+\operatorname{alpharad}(e q 2[4]))
$$

on Fig. 4.7 finally presents the waveform of the third harmonic where $2 * P i * e q 1[4] *$ $t 3=3 \omega t$ and alpharad is the phase in radiant.

Fig. G.1 utilizes the "IF - THEN" condition which is a more direct approach. Basically, the idea is to create an array which has all zeros but the desired component. Consider the equation

$$
w 1=I F \text { freq } E Q U A L S \text { freq[2] THEN vec[2] ELSE freq*0 ENDIF(G.3) }
$$


on Fig. G.1. Here the desired component is the fundamental. Eq. G.3 preserves only the fundamental spectral line, which is then displayed by $v e c 1=t s(w 1)$. The equation

$$
\text { check }=v e c 1+v e c 2+v e c 3+v e c 4
$$

on Fig. G.1 checks how closely the full frequency waveform is approximated by four harmonics. The agreement is not too bad but could be improved. Three harmonics

\begin{tabular}{|l|l|l|l|l|}
\hline & fund & & & Wweform of fund, sec., third \\
and fourth
\end{tabular}

Figure G.1: Approximation of a full frequency waveform by 4 harmonic frequencies. are certainly not enough to resemble the full frequency waveform which in turn 
means that the simulation producing Fig. 4.7 needs to consider more than three harmonics. But as mentioned before, as long as the magnitude is smaller than unity (which is the practical relevant case) 3 harmonics suffice. Both methods are equivalent. While the "IF - THEN" method appears handier the method involving "generate" explicitely displays the sinusoidal with magnitude and phase. It is worth mentioning that the phase changes slightly if more harmonics are taken into account i.e. $\alpha=-66.031^{\circ}$ and $\beta=54.547^{\circ}$. 


\section{Bibliography}

[1] T. Berceli. Nonlinear Active Microwave Circuits. Elsevier Science Publishing Co. Inc., 52 Vanderbilt Ave., New York, NY 10017, 1987.

[2] D.Pavlidis D.R. Pehlke, A. Samelis. Volterra series based nonlinear simulation of HBT's using analytically extracted models. Electronics Letters, vol. 30(no. 13):pp. 1098-1100, June, 23rd 1994.

[3] D. A. Teeter et al. Large-signal numerical and analytical HBT models. IEEE Transactions on Electron Devices, vol. 40(no. 5):pp. 837-845, May 1993.

[4] J. J. Bussgang et al. Analysis of nonlinear systems with multiple inputs. Proc. IEEE, vol. 62(no. 8):pp. 1088-1119, August 1974.

[5] N. L. Wang et al. AlGaAs/GaAS HBT linearity characteristic. IEEE Transactions on Microwave Theory and Techniques, vol. 42(no. 10):pp. 1845-1850, Oct. 1994.

[6] S. A. Maas et al. Intermodulation in Heterojunction Bipolar Transistors. IEEE Transactions on Microwave Theory and Techniques, vol. 40(no. 3):pp. 442 448, March 1992.

[7] T. J. Brazil et al. A new large-signal AlGaAs/GaAs HBT model including self-heating effects, with corresponding parameter-extraction procedure. IEEE Transactions on Microwave Theory and Techniques, vol. 43(no. 7):pp. 14331445, July 1995.

[8] G. Gonzales. Microwave Transistor Amplifiers. Prentice-Hall, Inc., Englewood Cliffs, New Jersey 07632, 1984.

[9] A. R. Hambley. Electronics, A Top-Down Approach to Computer-Aided Circuit Design. Macmillan Publishing Company, 113 Sylvan Ave., Englewood Cliffs, NJ 07632, 1994.

[10] HP, Hewlett Packard Company, HP EEsof Division, 1400 Fountaingrove Parkway, Santa Rosa, CA 95403. HP EEsof RF and Microwave Design Systems, Student's Guide and Training Manual, Release 6.01, 4. edition, Dec. 1994.

[11] F. Kouril and K.Vrba. Non-Linear and parametric circuits. Ellis Horwood Limited, Market Cross House, Cooper Street, Chichester, West Sussex, PO19 $1 \mathrm{~EB}$, England, 1988. 
[12] Y. L. Kuo. Distortion analysis of Bipolar Transistor Circuits. IEEE Transactions on Circuit Theory, vol. CT-20(no. 6):pp. 709-716, November 1973.

[13] A. Laser and D. Pulfrey. Reconciliation of methods for estimating fmax for microwave heterojunction transistors. IEEE Transactions on Electron Devices, vol. 38(no. 8):pp. 1685-1692, August 1991.

[14] S. A. Maas. Nonlinear microwave circuits. Artech House, Inc., 685 Canton Street, Norwood, MA 02062, 1988.

[15] S. Narayanan. Transistor distortion analysis using Volterra series representation. Bell System Technical Journal, pages 991-1024, May-June 1967.

[16] K. B. Niclas and D. L. Skoglund. Third-order intermodulation distortion in a K-band amplifier. Microwave Journal, pages 88 - 96, May 1995.

[17] P. A. Rizzi. Microwave Engineering, Passive Circuits. Prentice-Hall, Inc., Englewood Cliffs, New Jersey 07632, 1988.

[18] A. Samelis and D. Pavlidis. Mechanisms determining third order intermodulation distortion in AlGaAs/GaAS HBT's. IEEE Transactions on Microwave Theory and Techniques, vol. 40(no. 12):pp. 2374 - 2380, Dec. 1992.

[19] U. Schaper and B. Holzapfl. Analytical parameter extraction of the HBT equivalent circuit with T-like topology from measured S-parameters. IEEE Transactions on Microwave Theory and Techniques, vol. 43(no. 3):pp. 493-498, March 1995. 